\title{
Min-Max Theory for the Yang-Mills-Higgs Equations
}

\author{
Clifford Henry Taubes* \\ Department of Mathematics, University of California, Berkeley, CA 94720, USA
}

\begin{abstract}
In each monopole sector there exist an infinite number of finite energy solutions to the Prasad-Sommerfield limit of the SU(2) Yang-MillsHiggs equations on $\mathbb{R}^{3}$ whose energy is greater than any finite number.
\end{abstract}

\section{Contents}

A.1 Introduction . . . . . . . . . . . . . . . . . . . . . . . . . . . 473

A.2 Strategy for Convergence . . . . . . . . . . . . . . . . . . . . . . . 476

A.3 Terminology . . . . . . . . . . . . . . . . . . . . . . . . . . . . . . 482

A.4 The Variational Problem . . . . . . . . . . . . . . . . . . . . . . . . . 483

B.1 The Regularized Problem . . . . . . . . . . . . . . . . . . . . . . . . . . 486

B.2 Minimizing Sets for $\mathfrak{Q}^{\delta}$. . . . . . . . . . . . . . . . . . . . . . . . . . . . 488

B.3 Convergence for Min-Max for $\mathfrak{A}^{\delta}$. . . . . . . . . . . . . . . . . . . . . . 493

B.4 Final Arguments for $\mathfrak{H}^{\delta}$. . . . . . . . . . . . . . . . . . . . . . . . . . . . 496

C.1 A priori Estimates . . . . . . . . . . . . . . . . . . . . . . . . . . . . . 498

C.2 Exponential Decay Estimates . . . . . . . . . . . . . . . . . . . . . 502

C.3 Power Law Estimates for $\Phi$. . . . . . . . . . . . . . . . . . . . . . . . 505

C.4 Power Law Estimates for A . . . . . . . . . . . . . . . . . . . . . . . . . 508

D.1 The Stress Energy Identity . . . . . . . . . . . . . . . . . . . . 513

D.2 The Cluster Decomposition . . . . . . . . . . . . . . . . . . . 516

D.3 The Interaction of Clusters . . . . . . . . . . . . . . . . . . . . . . . . 519

D.4 The Convergence of Min-Max for $\mathfrak{A}$. . . . . . . . . . . . . . . . . . . . 526

E.1 The Neighborhood of the Moduli Space . . . . . . . . . . . . . . . . . . . 528

E.2 Min-Max, and Pointed Homotopy . . . . . . . . . . . . . . . . . . . . . . 537

\section{A.1. Introduction}

The differential equations of a classical gauge theory are, in many cases, the formal variational equations of a functional (the action) on a topologically non-trivial space. And so it was conjectured $[1,2]$ that Morse theory, or some weaker analog might be useful for establishing the existence of non-trivial solutions. There are

* National Science Foundation Postdoctoral Fellow in Mathematics 
standard conditions which, if satisfied, imply the relationship between the topology of the space and the critical points of a function on that space $[3,4]$; Condition $C$ of Palais-Smale is one such condition. However, the Yang-MillsHiggs functional on $\mathbb{R}^{3}$ does not satisfy any of these conditions.

One could conjecture for this functional, on the one extreme, that its behavior was akin to the harmonic map energy functional on the space of $C^{\infty}$ maps from $S^{2}$ to $S^{2}$. The harmonic map energy functional obtains its minimum on each of the countable number of path components of $\operatorname{Maps}\left(S^{2} ; S^{2}\right)$; these are the holomorphic and anti-holomorphic maps. But these local minima are its only critical points [5], in spite of the rich topological structure of the space $\operatorname{Maps}\left(S^{2} ; S^{2}\right)$.

On the other extreme, one could imagine that the Yang-Mills-Higgs functional behaved like a "good" Morse function, in spite of the failure of the Palais-Smale condition. Indeed, a non-minimal solution to the SU(2) Yang-Mills-Higgs equations on $\mathbb{R}^{3}$ which relates to $\pi_{1}$ of the function space is known to exist $[6,7]$.

This article will prove that the $S U(2)$ Yang-Mills-Higgs functional on $\mathbb{R}^{3}$, in the Bogomol'nyi-Prasad-Sommerfield limit behaves like a good Morse function (Theorem A.1.2). One consequence of this fact, which is also established here, is that in each path component of the function space (monopole sector), there exists an infinite number of gauge inequivalent solutions to the associated variational equations; in fact, an infinite number with action greater than any fixed number (Theorem A.1.3). The results in [8] imply that all of these new critical points are unstable ones for the action functional.

This article is to be considered as a sequel to [9], to which the reader will often be referred. The results in this article were announced in [10].

These SU(2) Yang-Mills-Higgs equations are a set of partial differential equations on $\mathbb{R}^{3}$ where the unknown is a pair $(A, \Phi)$, with $A$ being a connection on the principal SU(2) bundle $P=\mathbb{R}^{3} \times \operatorname{SU}(2)$ over $\mathbb{R}^{3}$, and with $\Phi$ being a section of the associated bundle, $\operatorname{Ad} P=\mathbb{R}^{3} \times \mathrm{SU}(2)$. The equations are

$$
\begin{gathered}
D_{A} * F_{A}+*\left[\Phi, D_{A} \Phi\right]=0, \\
D_{A} * D_{A} \Phi=0,
\end{gathered}
$$

subject to the boundary condition that $1-|\Phi| \in L^{6}\left(\mathbb{R}^{3}\right)$. Here, $F_{A}$ is the curvature of $A\left(\right.$ a section of $\left.\hat{2} T^{*} \otimes \operatorname{Ad} P\right), D_{A}$ is the exterior covariant derivative on $\Lambda T^{*} \otimes \operatorname{Ad} P$ that $A$ defines, $*: \Lambda T^{*} \rightarrow \Lambda T^{*}$ is the Euclidean, Hodge dual and $[\cdot, \cdot]$ is the graded bracket on $\Lambda T^{*} \otimes \operatorname{Ad} P$. The norm $|\cdot|$ on $\Lambda T^{*} \otimes A d P$ is the product metric; (the Euclidean metric on $\left.\Lambda T^{*}\right) \otimes\left[\right.$ the metric -2 trace $\mathbb{C}^{2}(\cdot, \cdot)$ on $\left.\mathrm{SU}(2)\right]$. The uninitiated are referred to [9, Sects. A.1-A.3] and [11, Chaps. 1 and 4] for details.

Equation (A.1.1) is the variational equation for the action functional,

$$
\mathfrak{U}(A, \Phi)=\frac{1}{2} \int_{\mathbb{R}^{3}}\left\{\left|F_{A}\right|^{2}(x)+\left|D_{A} \Phi\right|^{2}(x)\right\} d^{3} x .
$$

One is to consider $\mathfrak{U}$ as a functional on the set

$$
\mathfrak{C}=\left\{\text { Smooth } c=(A, \Phi): \mathfrak{U}(c)<\infty \quad \text { and } \quad 1-|\Phi| \in L^{6}\left(\mathbb{R}^{3}\right)\right\} .
$$

The set $\mathfrak{C}$ is topologized as in [9, Sect. B.1].

The space $\widetilde{C}$ is homotopically $\operatorname{Maps}\left(S^{2} ; S^{2}\right)$ [9, Proposition A.1.1]. It therefore has also a countable set of disjoint path components, $\left\{\boldsymbol{C}_{n}\right\}_{n \in \mathbb{Z}}$. D. Groisser has 
shown that for $(A, \Phi) \in \mathfrak{C}_{n}[12]$,

$$
n=\frac{1}{4 \pi} \int_{\mathbb{R}^{3}}\left\{-2 \operatorname{trace}_{\mathbb{C}^{2}}\left(F_{A} \wedge D_{A} \Phi\right)\right\} .
$$

The triangle inequality then implies that for each $n \in \mathbb{Z}$,

$$
\left.\mathfrak{U}\right|_{\mathfrak{C}_{n}} \geqq 4 \pi|n| .
$$

For $c \in \mathfrak{C}_{n}$, equality in Eq. (A.1.5) occurs if and only if $c=(A, \Phi)$ satisfies the Bogomol'nyi equations [13],

$$
F_{A}=\operatorname{sign}(n) * D_{A} \Phi
$$

Solutions to the Bogomol'nyi equations exist in each $\mathfrak{C}_{n}[14,11,15]$. For $n=0$, any $c=(A, \Phi)$ satisfying Eq. (A.1.6) has $F_{A} \equiv 0$ and $D_{A} \Phi \equiv 0$.

Each $\mathbb{C}_{n}$ is acted on continuously by a topological transformation group, the gauge group [9, Sect. 13.1],

$$
\mathfrak{G}=\left\{\text { smooth automorphisms of } \mathbb{R}^{3} \times \mathrm{SU}(2)\right\} \simeq C^{\infty}\left(\mathbb{R}^{3} ; \mathrm{SU}(2)\right)
$$

The functional $\mathfrak{A}$ is invariant under the action of $\mathfrak{6}$ and solutions to Eqs. (A.1.1) or (A.1.6) are transformed into solutions. The subgroup

$$
\mathfrak{G}_{0}=\{g \in \mathfrak{5}: g(x=0)=1 \in \mathrm{SU}(2)\}
$$

acts freely on $\mathfrak{C}$ and the functional $\mathfrak{A}$ descends to the quotient $\mathfrak{B}$. As $\mathfrak{G}_{0}$ is contractible, $\mathfrak{B}$ embeds in $\mathfrak{C}$ as

$$
\left.\mathfrak{B}=\left\{c=(A, \Phi) \in \mathbb{C}: A(x=0)=0 \quad \text { and } \quad x^{i} \frac{\partial}{\partial x^{i}}\right\lrcorner A=0\right\} .
$$

Here, and in Eq. (A.1.8), a product structure on $P=\mathbb{R}^{3} \times \mathrm{SU}(2)$ has been chosen. There is a residual $S U(2)$ action on $\mathfrak{B}$ which leaves $\mathfrak{A}$ invariant. It is convenient to reduce this $\mathrm{SU}(2)$ action to an $S^{1}$ action by constructing a new space, $\hat{\mathfrak{B}}$, which embeds in $\mathfrak{B}$ (hence in $\mathfrak{C}$ ) as the fiber of a fibration [9, Sects. A.1 and B.5],

$$
\hat{\mathfrak{B}} \rightarrow \mathfrak{B} \stackrel{\hat{n}}{\longrightarrow} S^{2} .
$$

The group SU(2) acts on $S^{2}$ via rotations, and the fibration in Eq. (A.1.10) is $\mathrm{SU}(2)$ equivariant. The $S^{1}$ subgroup of $S U(2)$ which fixes the north pole, $p \in S^{2}$, fixes $\hat{\mathfrak{B}}=\hat{n}^{-1}(p)$. Then $\mathfrak{B}$ is the orbit of $\hat{\mathfrak{B}}$ under $\mathrm{SU}(2)$. This structure and the map $\hat{n}$ are all explained in [9, Sect. B.5].

One consequence of the preceding discussion is that $\mathfrak{A}$ only "sees" the topology of $\hat{\mathfrak{B}}$ (and the $S^{1}$ equivariant topology, at that). This is convenient because $\hat{\mathfrak{B}}$ is well understood:

Theorem A.1.1 (Theorem A.1.1 of [9]). The space $\hat{\mathfrak{B}}$ is homotopically the space $\Omega\left(S^{2}\right)$ of base-point preserving maps from $S^{2}$ to $S^{2}$.

The topology of $\mathfrak{B}$ and the critical points of $\mathfrak{A}$ can be related by min-max theory (cf. [3]); of which a brief review follows. Let $M$ be a smooth manifold. A family, $\mathfrak{F}$, of non-empty compact subsets of $M$ is said to be homotopy invariant if for 
any continuous $\phi:[0,1] \times M \rightarrow M$ for which $\phi(0, \cdot)=$ identity, it is true that the condition $F \in \mathfrak{F}$ implies that $\phi(1, F) \in \mathfrak{F}$.

For example, let $\left\{H^{k}(M ; \mathbb{Z}), k \geqq 0\right\}$ be the (compactly supported) cohomology of $M$ with coefficients in $\mathbb{Z}$. Let $[z] \in H^{k}(M ; \mathbb{Z})$ and set

$$
\begin{aligned}
\mathfrak{F}=\mathfrak{F}([z])= & \{F \leqq M: \text { the inclusion map, } i: F \rightarrow M, \text { has the property } \\
& \text { that } \left.i^{*}[z] \neq 0 \in H^{k}(F, \mathbb{Z})\right\} .
\end{aligned}
$$

The family above is homotopy invariant.

Let $f: M \rightarrow[0, \infty)$ be a smooth function. To a homotopy invariant family, $\mathfrak{F}$, assign the number

$$
f_{\mathscr{F}}=\inf _{\boldsymbol{F} \in \mathfrak{F}} \sup _{c \in F} f(c) .
$$

For a "good" function $f$ on $M$ one should require that $f$ has a critical point at the critical value $f_{\mathfrak{F}}$.

The theorem below states that $\mathfrak{A}$ on $\hat{\mathfrak{B}}$ is good.

Theorem A.1.2. Let $\mathfrak{F}$ be a homotopy invariant family. There exists a solution to Eq. (A.1.1), $c \in \hat{\mathfrak{B}}$ with $\mathfrak{\mathfrak { U }}(c)=\mathfrak{U}_{\mathfrak{F}}$.

Theorem A.1.2 of [9] asserts that for each $n \in \mathbb{Z}$, the set

$\mathrm{Crit}_{n}=\left\{\mathfrak{U}_{\mathfrak{F}}: \mathfrak{F}\right.$ is a homotopy invariant family of compact subsets of $\left.\mathfrak{C}_{n}\right\}$

is an unbounded subset of $\mathbb{R}$. Thus,

Theorem A.1.3. For each $n \in \mathbb{Z}, E q$. (A.1.1) has an infinite number of solutions in $\hat{\mathfrak{B}}_{n}$ with action above any finite level.

Theorem A.1.2, suitably modified, also provides information about the topology of the spaces $\hat{\mathfrak{M}}_{n} \subset \hat{\mathfrak{B}}_{n}$ of solutions to Eq. (A.1.6). Of import here is an observation from [8] that a suitably defined hessian of $\mathfrak{U}$ at a nonminimal critical point in $\hat{\mathfrak{B}}_{n}$ must have index $|n|+1$ or larger.

Theorem A.1.4. For each $n$, the inclusion $\mathfrak{M}_{n} \subset \hat{\mathfrak{B}}_{n}$ induces an isomorphism of the pointed homotopy group $\pi_{l}(\cdot)$ for $l<|n|$; and an epimorphism for $l=|n|$.

Recently, S.K. Donaldson proved [16] the remarkable fact that for each $n \in \mathbb{Z}$, $\hat{\mathfrak{M}}_{n}$ is homeomorphic to the space of base point preserving, rational maps from $S^{2}$ to $S^{2}$ with degree $n$. Thus, Theorems A.1.1 and A.1.4 with [17] recover a theorem of G. Segal: The inclusion of this space of rational maps into $\Omega_{n}\left(S^{2}\right)$ (the space of all smooth, base point preserving maps of degree $n$ ) induces an isomorphism of $\pi_{l}(\cdot)$ for $l<|n|$ and an epimorphism on $\pi_{|n|}(\cdot)$.

The proof of Theorem A.1.2 requires the author to introduce to the calculus of variations a number of new techniques. For this reason, a long, detailed overview of the strategy and logical train of the proof is provided in Sect. A.2.

\section{A.2. Strategy for Convergence}

To understand why convergent min-max sequences exist for $\mathfrak{A}$ on $\hat{\mathfrak{B}}$, it is necessary to understand first where the standard Palais-Smale Condition $C$ fails. Condition 
$C$ is satisfied by a smooth function, $f: M \rightarrow[0, \infty)$ on a smooth Banach manifold $M$ if the following statement is true: Every sequence $\left\{c_{i}\right\} \subset M$ for which $\left\{f\left(c_{i}\right)\right\}$ is bounded and for which $\left\{\left\|d f_{c_{i}}\right\|_{*}\right\}$ has limit zero has, itself, a convergent subsequence. Here, $\|\cdot\|_{*}$ is the norm on $T^{*} M$. (See $[3,4]$.)

Were Condition $C$ satisfied by $\mathfrak{A}$ on $\mathfrak{B}$, then Theorem A.1.2 would be practically a standard result [3]. The technical problems in the proof arise because Condition $C$ is not present due to the non-compactness of $\mathbb{R}^{3}$ (no Rellich lemma).

But, Condition $C$ is not the last word. Even a function on the real line, $\mathbb{R}$, may be a good Morse function (in the sense that its critical points determine a $C W$ decomposition of $\mathbb{R}$ (cf. [18]) but not satisfy Condition $C$. Consider, for example, the function $\phi$ on $\mathbb{R}$ which sends $t$ to $\phi(t)=t^{2} /\left(1+t^{2}\right)$. Observe that $d \phi=2 t d t /\left(1+t^{2}\right)^{2}$. Thus, $\phi$ has only one critical point, $t=0$, and there, the hessian $\partial^{2} \phi / \partial t^{2}$ is positive. This is the expected behavior of a good Morse function. However, consider the sequence of integral points, $\mathbb{Z} \subset \mathbb{R}$. It has the property that for each $k \in \mathbb{Z}, \phi(k)<1$, and as $|k| \rightarrow \infty,|d \phi(k)| \rightarrow 0$. But the sequence of integral points has no limit point in $\mathbb{R}$. Thus, $\phi$ does not satisfy Condition $C$.

The important principle here is the following: To prove that the critical points of a function $f: M \rightarrow[0, \infty)$ mirror the topology of $M$, only sequences $\left\{c_{i}\right\}$ for which $\left\{f\left(c_{i}\right)\right\}$ is decreasing need be considered.

Min-max theory for a function $f$ on a Banach manifold $M$ illustrates this principle. For example, let $\mathfrak{F}$ be a homotopy invariant family of compact subsets of $M$. One can try to establish that $f_{\mathfrak{F}}$ of Eq. (A.1.9) is a critical value of $f$ by considering min-max sequences in

$$
Y(\mathfrak{F}, f)=\left\{(F, c) \in \mathfrak{F} \times M: c \in F \quad \text { and } \quad f(c)=\sup _{F} f\right\} .
$$

A min-max sequence $\left\{\left(F_{i}, c_{i}\right)\right\} \subset Y$ is one for which the $\left\{f\left(c_{i}\right)\right\}$ are decreasing to $f_{\mathfrak{F}}$ :

$$
f\left(c_{i}\right) \geqq f\left(c_{i+1}\right) \rightarrow f_{\mathfrak{F}} .
$$

The pertinent question is: Can a min-max sequence $\left\{\left(F_{i}, c_{i}\right)\right\} \subset Y$ be chosen for which $\left\{c_{i}\right\}$ converges in $M$ to a critical point of $f$ ?

It can be remarked that this question is subtle; required for the answer is a detailed knowledge of the function involved. For example, when $M=\mathbb{R}$, one must distinguish between the functionals $\left\{f^{\delta}(\cdot): \delta \in[0,1]\right\}$ given by

$$
f^{\delta}(t)=e^{t}+\frac{\delta t}{\left(1+t^{2}\right)}
$$

For $\delta=0, f^{0}(\cdot)$ is not a good Morse function on $\mathbb{R}$ as it has no critical points. But for any $\delta>0$, and in spite of Condition $C$ 's failure, $f^{\delta}(\cdot)$ is a good Morse function on $\mathbb{R}$.

For the Yang-Mills-Higgs problem, K. Uhlenbeck's compactness theorems in [19] imply that a sequence $\left\{c_{i}\right\} \subset \hat{\mathfrak{B}}$ can converge only if the sequence $\left\{\mathfrak{U}\left(c_{i}\right)\right\}$ is bounded; and only if the curvatures $\left\{\left(F_{A}, D_{A} \Phi\right): c_{i}=(A, \Phi)\right\}$ have sufficiently uniform decay as $|x| \rightarrow \infty$ on $\mathbb{R}^{3}$, independent of the index $i$.

This fact motivates the study as a function of $c=(A, \Phi) \in \hat{\mathfrak{B}}$ of the maximum separation of the sets in $\mathbb{R}^{3}$ where $\left|F_{A}\right|^{2}(x)+\left|D_{A} \Phi\right|^{2}(x)$ is large. One assigns to each $c \in \mathfrak{B}$ a number $\varrho(c)$ which describes this maximum separation. To be precise, the 
number $\varrho(c)$ is defined in the following way: One considers the local action of $c$ in unit balls; this is a map $\mathfrak{s}: \hat{\mathfrak{B}} \rightarrow C^{0}\left(\mathbb{R}^{3}\right)$ given by

$$
\mathfrak{s}(c)(x)=\frac{1}{2} \int_{|x-y|<1} d^{3} y\left\{\left|F_{A}\right|^{2}+\left|D_{A} \Phi\right|^{2}\right\} .
$$

For a suitably chosen $\kappa>0$, one defines

$$
\hat{U}(c)=\left\{x \in \mathbb{R}^{3}: \mathfrak{s}(c)(x)>\kappa\right\} \quad \text { and } \quad U(c)=\left\{x \in \mathbb{R}^{3}: \operatorname{dist}(x, \hat{U}(c))<1\right\} .
$$

On $\mathbb{R}^{3} \backslash U(c)$, the curvatures are sufficiently small so that they are effectively linear in the basic field $c=(A, \Phi)$. On $U(c)$, the curvatures are large and the nonlinearities play the crucial role.

Observe that the set $U(c)$ cannot be arbitrarily bad. Indeed, it is bounded, and it has at most $\mathfrak{U}(c) / \kappa$ path components, and the sum of the diameters of the path components is at most $4 \mathfrak{U}(c) / \kappa$ (see Lemma C.1.2).

The map $\varrho(\cdot): \hat{B} \rightarrow[0, \infty)$ assigns to $c$ the maximum separation of the path components of $U(c)$ [Eq. (D.1.1)].

It is crucial to realize that a sequence $\left\{c_{i}\right\} \subset \mathfrak{B}$ can converge in $\mathfrak{B}$ only if the sequences $\left\{\varrho\left(c_{i}\right)\right\}$ and $\left\{\mathfrak{U}\left(c_{i}\right)\right\}$ converge in $[0, \infty)$ (see Sects. C.4 and D.4).

Let $\mathfrak{F}$ be a homotopy invariant family of compact subsets of $\mathfrak{B}$. Then one must obtain min-max sequences $\left\{\left(F_{i}, c_{i}\right)\right\} \subset Y(\mathfrak{F})$ for which the sequence $\left\{\varrho\left(c_{i}\right)\right\}$ is bounded; whether or not they exist is a delicate question. To motivate the proof of their existence, consider the following construction: By assigning to each $c \in \hat{\mathfrak{B}}$ the set of centers of the path components of $U(c)$, one obtains a point $\Psi(c)$ in one of the configuration spaces $C_{l}\left(\mathbb{R}^{3}\right)$, for $l \in\{0,1, \ldots,[\mathfrak{U}(c) / \kappa]\}$. Here, $C_{l}\left(\mathbb{R}^{3}\right)$ is the space of $l$-tuples of distinct points in $\mathbb{R}^{3}$. Thus, $\Psi$ defines a map from $\hat{B}$ into $\bigcup_{l=0}^{\infty} C_{l}\left(\mathbb{R}^{3}\right)$ with the property that for any $E \in[0, \infty), \Psi$ maps $\mathfrak{A}^{-1}([0, E))$ into the finite dimensional space $\bigcup_{l=0}^{E / \kappa} C_{l}\left(\mathbb{R}^{3}\right)$. One may reinterpret the min-max problem for $\mathfrak{F}$ as that of finding a min-max sequence $\left\{\left(F_{i}, c_{i}\right)\right\} \subset Y(\mathfrak{F})$ such that the sequence of points $\left\{\Psi\left(c_{i}\right)\right\} \subset \bigcup_{l=0}^{\Lambda} C_{l}\left(\mathbb{R}^{3}\right), \Lambda=\left[2 \mathfrak{A}_{\widetilde{\mho}} / \kappa\right]$, is bounded.

Because the noncompactness here reflects the noncompactness of $C_{l}\left(\mathbb{R}^{3}\right)$, it is enlightening to consider a model variational problem on $C_{l}\left(\mathbb{R}^{3}\right)$ itself. One can imagine a physical system of $l$-particles on $\mathbb{R}^{3}$ which interact via pair potentials. Then the energy of a point $\left\{x_{1}, \ldots, x_{l}\right\} \in C_{l}\left(\mathbb{R}^{3}\right)$ is given by

$$
\mathfrak{E}\left(\left\{x_{1}, \ldots, x_{l}\right\}\right)=\text { constant }+\sum_{i<j} v\left(x_{i}-x_{j}\right) .
$$

Here, $v(\cdot) \in C^{\infty}\left(\mathbb{R}^{3}\right)$ is the potential. If $v$ has the property that for some $x \neq 0$,

$$
\lim _{t \rightarrow \infty} v(t x)<\infty,
$$

then $\mathfrak{E}$ may not satisfy Condition $C$ on $C_{l}\left(\mathbb{R}^{3}\right)$. However, if for all $x \neq 0$, and $t$ sufficiently large,

$$
v(t x)<\lim _{s \rightarrow \infty} v(s x)
$$


then one expects that the function $\mathbb{E}$ is really a good Morse function on $C_{l}\left(\mathbb{R}^{3}\right)$ because Eq. (A.2.2) says that it costs energy to pull two particles apart [of course, $v(\cdot)$ must be repulsive for small $|x|]$.

The analysis above suggests that one should try to write the action $\mathfrak{A}\left(c_{i}\right)$ for each $c_{i}$ of a min-max sequence $\left\{\left(F_{i}, c_{i}\right)\right\} \subset Y(\mathscr{F})$ as a sum of two parts: The first part is the contribution from each path component of $U\left(c_{i}\right)$ and the second is the contribution from $\mathbb{R}^{3} \backslash U\left(c_{i}\right)$. The contribution from $\mathbb{R}^{3} \backslash U\left(c_{i}\right)$ one wants to interpret as an "interaction energy" between the path components of $U\left(c_{i}\right)$ and then ask whether it costs energy to separate the path components. The analysis would then proceed, at least heuristically, as if the variational problem were on some $C_{l}\left(\mathbb{R}^{3}\right)$.

This procedure is feasible if one can obtain sufficiently strong a priori estimates for the curvatures of each $c_{i}$ in the small field region, $\mathbb{R}^{3} \backslash U\left(c_{i}\right)$. The existence of a priori estimates is due to the fact that the Yang-Mills-Higgs equations are semilinear and $\mathbb{R}^{3} \backslash U\left(c_{i}\right)$ is, by definition, precisely the region where the linear approximation holds. Indeed, one might expect that for $i$ large, $c_{i}$ is approximately a solution to Eq. (A.1.1). For an exact solution, $c$, to Eq. (A.1.1), the fields in the region $\mathbb{R}^{3} \backslash U(c)$ satisfy to order $\exp (-\operatorname{dist}(x, U(c))$ the linearized equations in $\mathbb{R}^{3} \backslash U(c)$ with some boundary conditions on $\partial U(c)$ (Sects. C.1-C.4; [24] and [11, Chap. IV]). This just states the fact, long accepted by physicists, that from far away, a non-abelian monopole looks abelian (i.e., a Dirac monopole [21]).

The trick is to obtain min-max sequences $\left\{\left(F_{i}, c_{i}\right)\right\}$ for $\mathfrak{F}$ such that each $c_{i}$ essentially satisfies the a priori estimates in $\mathbb{R}^{3} \backslash U\left(c_{i}\right)$ of a solution to Eq. (A.1.1). The author has two methods for obtaining min-max sequences which satisfy such detailed estimates. The less elegant procedure is technically easier. It involves constructing a sequence of regularized functionals $\left\{\mathfrak{H}^{\delta}: \delta \in\left[0, \frac{1}{2}\right)\right\}$ on $\mathfrak{B}$ (with $\left.\mathfrak{A}^{0}=\mathfrak{A}\right)$ :

$$
\mathfrak{U}^{\delta}(A, \Phi)=\frac{1}{2} \int_{\mathbb{R}^{3}}\left\{\left(1+|x|^{2}\right)^{\delta}\left|F_{A}\right|^{2}(x)+\left|D_{A} \Phi\right|^{2}(x)\right\} d^{3} x .
$$

The set $\left\{\mathfrak{Q}^{\delta} ; \delta \in\left[0, \frac{1}{2}\right)\right\}$ has the following properties (Lemma B.1.2):

(1) If $\delta>\delta^{\prime}$, then $\mathfrak{r}_{\mathscr{\gamma}}^{\delta} \geqq \mathfrak{H}_{\mathscr{\delta}}^{\delta^{\prime}}$, and

(2) $\lim _{\delta \rightarrow 0} \mathfrak{H}_{\mathfrak{F}}^{\delta}=\mathfrak{A}_{\mathfrak{F}}$.

Of crucial import is that for any $\delta \in\left(0, \frac{1}{2}\right)$ and any homotopy invariant family $\mathbb{\&}$ of $\mathfrak{B}$, there exist min-max sequences $\left\{\left(F_{i}^{\delta}, c_{i}^{\delta}\right)\right\} \subset Y^{\delta}(\mathfrak{F})$ for $\mathfrak{H}^{\delta}$ such that $\left\{c_{i}^{\delta}\right\}$ converges in $\hat{\mathfrak{B}}$ to $c^{\delta}(\mathfrak{F})$, a solution of the variational equations of $\mathfrak{H}^{\delta}$ with $\mathfrak{U}^{\delta}\left(c^{\delta}(\mathfrak{F})\right)=\mathfrak{H}_{\mathfrak{F}}^{\delta}$. This fact is established as Proposition B.1.3.

The variational equations for $\mathfrak{H}^{\delta}$ are used to prove that each $c^{\delta}(\mathfrak{F})$ obeys the required estimates (see Sects. C.1-C.4). The techniques for this task were developed in [24], see also [11]. The necessary min-max sequences for $\mathfrak{A}$ can be constructed from a diagonal subsequence of the doubly indexed sequence $\left\{\left(F_{i}^{\delta}, c_{i}^{\delta}\right): i \in\{1, \ldots\}\right.$ and $\left.\delta \in\left(0, \frac{1}{2}\right)\right\}$.

The alternate method for obtaining a priori estimates for min-max sequences involves deforming $(F, c) \in Y(\mathfrak{F})$ to $\left(F^{\prime}(F), c^{\prime}\right)$, with $F^{\prime}(b)$ obtained from $b \in F$ by solving Eq. (A.1.1) in the small field region, $\mathbb{R}^{3} \backslash U(b)$ (where these equations are practically linear). This procedure is technically complicated, but it has applica- 
tions to other elliptic, semilinear variational problems which are borderline for Condition $C$, and it will be discussed in a forthcoming article, not here.

For each $c^{\delta}(\mathfrak{F})(\delta>0)$, the fields in the small field region are determined via an essentially linear system of equations by their values on $\partial U\left(c^{\delta}(\mathfrak{F})\right)$. This fact allows the action, $\mathfrak{U}^{\delta}\left(c^{\delta}(\mathfrak{F})\right)$, to be decomposed into its $U\left(c^{\delta}(\mathfrak{F})\right)$ contribution, and the remainder. This remainder is to leading order the interaction energy of a system of point particles, one in each path component of $U\left(c^{\delta}(\mathfrak{F})\right)$ which interact via longrange fields whose equations are the linearized Yang-Mills-Higgs equations with a delta-function source at each particle's position. The strength of the delta function is determined by the boundary values of $c^{\delta}(\mathfrak{F})$ on the path component of $U\left(c^{\delta}(\mathfrak{F})\right.$ ) which contains that particle. This is pure potential theory for what is essentially Laplace's equation in an exterior domain. The technical lemmas that establish this picture of $c^{\delta}(\mathfrak{F})$ are proved in Sects. D.1-D.3.

With these a priori estimates established, one could proceed by a reductio ad absurdum argument along the following lines: suppose that the linearized equations provide, via potential theory, an interaction energy which defines attractive forces between the path components of $U\left(c^{\delta}(\mathfrak{I})\right)$. Now assume that the maximum separation of the path components was large. In this case, one could construct by "cutting and pasting" a one-parameter family of configurations that started from $c^{\delta}(\mathfrak{F})$ and which amounted to a rigid translation of the far-flung path components of $c^{\delta}(\mathfrak{F})$ towards each other. The attractive forces would insure that $\mathfrak{U}^{\delta}$ was not stationary at $c^{\delta}(\mathfrak{F})$ along such a one-parameter family.

In fact, an actual deformation of $c^{\delta}(\mathfrak{F})$ is not required. A critical point $c=(A, \Phi)$ of $\mathfrak{A}^{\delta}$ satisfies a "virial theorem" that is derivable from the differential equation (see Corollary D.1.5 and [11, Chap. 2]). This virial theorem is the following integral equality:

$$
\int_{\mathbb{R}^{3}} d^{3} y\left\{\left|D_{A} \Phi\right|^{2}-\left(1-2 \delta|y|^{2}\left(1+|y|^{2}\right)^{-1}\right)\left(1+|y|^{2}\right)^{\delta}\left|F_{A}\right|^{2}\right\}=0 .
$$

A uniform bound on $\varrho(c)$ comes from Eq. (A.2.4). It is a side remark that this equation can be obtained by choosing a suitable deformation of $c$ and using the fact that $c$ is a critical point of $\mathfrak{A}^{\delta}$. Equation (A.2.4) provides a short-cut which avoids the necessity of constructing the actual deformation.

To utilize Eq. (A.2.4), one evaluates the integral by computing the contributions from the large field regions separately from the weak field regions. The a priori estimates and Eq. (A.2.4) yield the following inequality (Proposition D.3.2):

$$
0 \geqq \sum_{\mu, \lambda}^{\prime} \frac{(\mathfrak{a}(\mu) \mathfrak{a}(\lambda)-\mathfrak{n}(\mu) \mathfrak{n}(\lambda))}{\operatorname{dist}(\mu, \lambda)}+\delta \kappa_{0}\left(\mathfrak{U}^{\delta}(c)\right)-\zeta\left(\mathfrak{U}^{\delta}(c)\right) \varrho(c)^{-3 / 2} .
$$

Here, the primed summation is over suitably defined pairs $(\mu, \lambda)$ of clusters of path components of $U(c)$ which are mutually separated by $\operatorname{dist}(\mu, \lambda)=\mathcal{O}(\varrho(c))$ (see Sect. D.2). The functions $\kappa_{0}(\cdot)$ and $\zeta(\cdot)$ are positive and continuous in their arguments. The number $\mathfrak{a}(\mu)$ is an action associated to the cluster $\mu$; Lemma D.2.2 makes this precise, but essentially,

$$
\mathfrak{a}(\mu) \doteq \frac{1}{8 \pi} \int_{\mu} d^{3} x\left(\left(1+|x|^{2}\right)^{\delta}\left|F_{A}\right|^{2}+\left|D_{A} \Phi\right|^{2}\right)
$$


The number $\mathfrak{n}(\mu)$ is the local "monopole number" of the cluster $\mu$; essentially

$$
\mathrm{n}(\mu) \doteq \frac{1}{4 \pi} \int_{\mu} d^{3} x\left(F_{A}, * D_{A} \Phi\right) .
$$

Equation (A.2.4) has an analogy in the aforementioned model problem of $l$-particles on $\mathbb{R}^{3}$ which interact via the pair-wise potential $v\left(x_{i}-x_{j}\right)$. For a critical configuration of $l$-particles at positions $\left\{x_{1}, \ldots, x_{l}\right\}$ in $\mathbb{R}^{3}$, the corresponding virial theorem is

$$
\sum_{i<j} \sum_{\alpha=1}^{3}\left(x_{i}-x_{j}\right)^{\alpha}\left(\frac{\partial}{\partial x^{\alpha}} v\right)\left(x_{i}-x_{j}\right)=0 .
$$

Equation (A.2.7) is derivable by using the fact that the energy for a critical configuration, $\left\{x_{1}, \ldots, x_{l}\right\}$, is stationary at $t=1$ along the curve $t \mapsto\left\{t x_{1}, \ldots, t x_{l}\right\}$.

As an aside, the pair potential, $v(x(\mu)-x(\lambda)$ ), whose virial identity, Eq. (A.2.7), is exactly the first term in Eq. (A.2.5) is the Coulomb potential,

$$
v(x(\mu)-x(\lambda))=-\frac{(\mathfrak{a}(\mu) \mathfrak{a}(\lambda)-\mathfrak{n}(\mu) \mathfrak{n}(\lambda))}{|x(\mu)-x(\lambda)|} .
$$

This potential describes the "force" between far separated path components of $U(c)$. Such an interaction potential is precisely what physicists have expected for monopoles in the Prasad-Sommerfield limit [20,21].

Examine Eq. (A.2.5). Without the interaction term (the first term), the second and third terms provide a $\delta$-dependent bound on $\varrho(c)$. Such is to be expected, since the weight $\left(1+|x|^{2}\right)^{\delta}$ in $\mathfrak{U}^{\delta}$ is designed expressly to keep the path components of $U(c)$ together.

If all the "charges" in Eq. (A.2.5) satisfy

$$
\mathfrak{a}(\mu) \mathfrak{a}(\lambda)-\mathfrak{n}(\mu) \mathfrak{n}(\lambda) \geqq \mathcal{O}\left(\delta, \varrho^{-1 / 2}\right) ;
$$

and if at least one such charge is greater than some $\varepsilon>0$; then Eq. (A.2.5) provides an $\delta$-independent bound on $\varrho(c)$ from its first and third terms, viz:

$$
\varrho(c) \leqq \zeta^{\prime}\left(\mathfrak{A}^{\delta}(c)\right) \cdot \varepsilon^{-2} .
$$

Here, $\zeta^{\prime}(\cdot)$ is a continuous function of its argument. It is in this case that the intercluster forces are attractive. Thus it is of crucial importance to obtain information about the numbers $\{\mathfrak{a}(\mu), \mathfrak{n}(\mu)\}$ which appear in Eq. (A.2.5).

The essential properties of the $\{\mathfrak{a}(\mu), \mathfrak{n}(\mu)\}$ are derived in Sect. D.2. These properties are:

$$
\begin{gathered}
\mathfrak{a}(\mu) \geqq \kappa+\mathcal{O}\left(\delta, \varrho^{-1 / 2}\right), \\
|\mathfrak{n}(\mu)| \leqq \mathfrak{a}(\mu)+\mathcal{O}\left(\delta, \varrho^{-1 / 2}\right), \\
4 \pi \sum_{\mu} \mathfrak{a}(\mu)=\mathfrak{A}^{\delta}(c)+\mathcal{O}\left(\delta, \varrho^{-1 / 2}\right), \\
\sum_{\mu} \mathfrak{n}(\mu)=n(c)+\mathcal{O}\left(\delta, \varrho^{-1 / 2}\right),
\end{gathered}
$$


where $n(c)$ is the "monopole number" from Eq. (A.1.4) (see Lemmas D.2.2 and D.2.3). Given Eq. (A.2.6), Eq. (A.2.11) is not surprising.

Observe that Eq. (A.2.11.2) implies Eq. (A.2.9). Further, if Eq. (A.2.9) is an equality for all pairs of clusters $(\mu, \lambda)$, then Eq. (A.2.11.2) implies that each cluster has $\mathfrak{a}(\mu)=\ln (\mu) \mid+\mathcal{O}\left(\delta, \varrho^{-1 / 2}\right)$ and that all the $\mathfrak{n}(\mu)$ 's have the same sign. In this eventuality, Eqs. (A.2.11.3-4) force the critical value to satisfy

$$
\mathfrak{U}^{\delta}(c)=4 \pi|n(c)|+\mathcal{O}\left(\delta, \varrho^{-1 / 2}\right) .
$$

To see the consequences of Eq. (A.2.5), let $\mathfrak{F}$ be a homotopy invariant family of compact subsets of $\mathfrak{C}_{n}$ for some integer $n$. Let $\left\{c^{\delta}(\mathfrak{F}) ; \delta \in(0,1 / 2)\right\}$ be the set of critical points of $\mathfrak{U}^{\delta}$ with critical values $\mathfrak{U}_{\mathscr{\gamma}}^{\delta}$. Two possibilities arise. The first is that the number $\mathfrak{U}_{\mathfrak{F}}>4 \pi|n|$. Here, for $\delta$ sufficiently small, Eq. (A.2.11) provides at least one pair $(\mu, \lambda)$ in the first term of Eq. (A.2.5) for which

$$
\mathfrak{a}(\mu) \mathfrak{a}(\lambda)-\mathfrak{n}(\mu) \mathfrak{n}(\lambda) \geqq \zeta\left(\mathfrak{U}_{\mathfrak{\mho}}\right)\left(\mathfrak{U}_{\mathfrak{\mho}}-4 \pi|n|\right)+\mathcal{O}\left(\delta, \varrho^{-1 / 2}\right) .
$$

Here, $\zeta(\cdot)$ is a strictly positive, continuous function of its argument. Thus, if $\mathfrak{U}_{\mathfrak{F}}>4 \pi|n|$, an a priori bound on $\varrho\left(c^{\delta}(\mathfrak{F})\right)$ for $\delta>0$ is provided by Eq. (A.2.13) and Eq. (A.2.10) (with $\varepsilon=\mathfrak{U}_{\mathscr{F}}-4 \pi|n|$ ). See Proposition D.1.1 and the beginning of Sect. D.3. When $\mathfrak{U}_{\mathfrak{F}}>4 \pi|n|$, the proof of Theorem A.1.2 is completed in Sect. D.4.

In the case where $\mathfrak{U}_{\mathfrak{F}}=4 \pi|n|$, Eq. (A.2.5) provides no a priori bound on $\varrho\left(c^{\delta}(\mathfrak{F})\right.$ ). This is no surprise because the moduli spaces of solutions to Eq. (A.1.6) are known to be noncompact; in fact, these contain configurations which have large field regions with arbitrary separation $[11,14]$. Theorem A.1.1 is proved in the case $\mathfrak{U}_{\mathfrak{F}}=4 \pi|n|$ in Sect. E.1, where it is shown (Proposition E.1.1) that for each $n \in \mathbb{Z}$, positive $\varepsilon$ exists such that $\mathfrak{A}^{-1}([4 \pi|n|, 4 \pi|n|+\varepsilon)) \cap \hat{\mathfrak{B}}_{n}$ retracts onto $\hat{\mathfrak{M}}_{n}$.

As for Theorem A.1.4, it is practically a corollary to Theorem A.1.2 and Theorem 1.2 of [8]. The theorem requires the Yang-Mills-Higgs analog of a result in Sect. 5 of [22] which establishes the role of the Hessian of $\mathfrak{A}$ in the min-max procedure. The details appear in Sect. E.2.

\section{A.3. Terminology}

Most of the terminology in this article has been established in Sect. A.3 of [9]. However, certain conventions are new here. Let $P=\mathbb{R}^{3} \times \mathrm{SU}(2)$. Implicit is a fixed product structure on $P$. Thus a pair $(A, \Phi) \in \mathbb{C}$ is a pair (connection on $P$, section of $\operatorname{Ad} P$ ), but also a pair (su(2) valued 1 -form on $\mathbb{R}^{3}$, map from $\mathbb{R}^{3}$ into $\mathfrak{s u}(2)$ ), where $\mathfrak{s u}(2)=$ Lie Alg SU(2). The topology on $\mathbb{C}$ is induced by the map of $\mathbb{C}$ into $\times C_{12}^{\infty}\left(\mathbb{R}^{3}\right) \times[0, \infty)$ which sends $(A, \Phi)$ to components of $A$ and $\left.\Phi\right\} \times \mathfrak{U}(A, \Phi)$. The statement that a sequence converges in $\mathfrak{C}$ or $\hat{\mathfrak{B}}$ will always imply convergence in the above topology. As a tool to prove such convergence, an auxiliary topology is required. This is the $L_{1 ; \text { loc }}^{2}$ topology. A sequence $\left\{\left(A_{i}, \Phi_{i}\right)\right\} \in \mathbb{C}$ is said to converge strongly in the $L_{1 ; \text { loc }}^{2}$ topology to $(A, \Phi) \in \mathbb{C}$ if the sequence of numbers $\left\{\mathfrak{U}\left(A_{i}, \Phi_{i}\right)\right\}$ converges to $\mathfrak{A}(A, \Phi)$ and if on any bounded domain $\Omega \subset \mathbb{R}^{3}$, the sequence $\left\{\left.\left(A_{i}, \Phi_{i}\right)\right|_{\Omega}\right\}$ converges strongly in the $L_{1}^{2}$-Sobolev topology to $\left.(A, \Phi)\right|_{\Omega}$. This is to say 
that

$$
\lim _{i \rightarrow \infty} \int_{\Omega} d^{3} y\left\{\left|\nabla\left(A-A_{i}\right)\right|^{2}+\left|\left(A-A_{i}\right)\right|^{2}\right\}=0,
$$

and similarly for $\left(\Phi-\Phi_{i}\right)$. Here, $\nabla$ denotes the flat connection on $\mathbb{R}^{3} \times \operatorname{SU}(2)$. Notice that if $\left\{\left(A_{i}, \Phi_{i}\right)\right\}$ is in $\mathbb{C}_{n}$ and converges in the $L_{1 ; \operatorname{loc}}^{2}$ topology on $\mathfrak{C}_{n}$ to $(A, \Phi) \in \mathbb{C}$, then $(A, \Phi) \in \mathfrak{C}_{n}$. This is a consequence of Eq. (A.1.4) and Proposition B.3.1 of [9].

Certain terminology appears throughout the article, and should be introduced. The vector bundle $\operatorname{Ad} P \otimes\left(T^{*} \oplus \mathbb{R}\right)$ over $\mathbb{R}^{3}$ is denoted by $Q$. If $E \rightarrow \mathbb{R}^{3}$ is any vector bundle, $\Gamma_{0}(E)=$ Space of $C^{\infty}$, compactly supported sections of $E$, and $\Gamma(E)=$ Space of $C^{\infty}$ sections of $E$. If $E$ is associated to $\mathbb{R}^{3} \times \mathrm{SU}(2)$ by a representation $\varrho$, and if $A$ is a connection on $\mathbb{R}^{3} \times \mathrm{SU}(2)$, then $\nabla_{A}: \Gamma(E) \rightarrow \Gamma\left(E \otimes T^{*}\right)$ denotes the induced covariant derivative on $\Gamma(E)$. A flat, $\mathrm{SU}(2)$ invariant fiber metric on $E$ is denoted $(\cdot, \cdot)$. The Euclidean metric on $T^{*}$ is the only one considered. The fiber metric on $E$ induces the $L^{2}$-metric, $\langle\cdot, \cdot\rangle_{2}$ on $\Gamma_{0}(E)$ and the $L^{p}$-norms $\|\cdot\|_{p}$. The formal $L^{2}$-adjoint of $\nabla_{A}$ is denoted by $\nabla_{A}^{*}$.

An origin in $\mathbb{R}^{3}$ is fixed as are a set of Cartesian coordinates, $\{x\}$. It is necessary to have available a $C^{\infty}$-bump function, $\beta \in C^{\infty}([0, \infty) ;[0,1])$ which is identically 1 on $\left[0, \frac{1}{2}\right]$ and identically zero on $\left[\frac{3}{4}, \infty\right)$. This $\beta$ will also be considered as a function on $\mathbb{R}^{3}$ via the assignment $x \in \mathbb{R}^{3} \mapsto \beta(|x|)$. For $r \in(0, \infty), \beta_{r}(x)=\beta(x / r)$.

Finally, an important convention concerning constants is strictly adhered to. The letter $z$ will denote a numerical constant which is independent of any external parameters unless explicitly noted. In any given derivation, the precise value of $z$ may change from line to line. Similarly, the letter $\zeta$ will always denote a continuous function from $[0, \infty)$ to $(0, \infty)$ which is also independent of any external parameters unless explicitly noted. The precise function may change from line to line.

\section{A.4. The Variational Problem}

Equation (A.2.3) defines a functional $\mathfrak{A}^{\delta}$ for each $\delta \in\left[0, \frac{1}{2}\right)$ whose domain is the subset

$$
\mathfrak{C}^{\delta}=\left\{c \in \mathbb{C}: \mathfrak{A}^{\delta}(c)<\infty\right\} .
$$

According to the discussion in Sect. B.2 of [9], for no $n \in \mathbb{Z}$ and $\delta \in\left(0, \frac{1}{2}\right)$ is $\mathfrak{C}_{n}^{\delta}=\mathfrak{C}^{\delta} \cap \mathfrak{C}_{n}$ empty.

As $\mathfrak{A}^{\delta}$ is $\mathfrak{G}$ invariant, the construction in Sect. B.5 of [9] produces for each $\delta \in\left[0, \frac{1}{2}\right)$, spaces $\hat{\mathfrak{B}}^{\delta}=\hat{\mathfrak{B}} \cap \mathfrak{C}^{\delta}$, and for each $n \in \mathbb{Z}, \hat{\mathfrak{B}}_{n}^{\delta}=\hat{\mathfrak{B}}_{n} \cap \mathfrak{C}^{\delta}$. Section B.2 of [9] defines a topology on $\mathfrak{C}^{\delta}$ for which each $\mathfrak{C}_{n}^{\delta}$ is homotopic to $\mathfrak{C}_{n}$ and each $\hat{\mathfrak{B}}_{n}^{\delta}$ is homotopic to $\mathfrak{B}_{n}$. By design, the function $\mathfrak{A}^{\lambda}$ on $\mathfrak{C}^{\delta}$ or $\mathfrak{B}^{\delta}$ is continuous whenever $\frac{1}{2}>\delta \geqq \lambda \geqq 0$.

In order to discuss the calculus of variations on $\hat{\mathfrak{B}}^{\delta}$, the notion of differentiation is required: 
Definition A.4.1. Let $\delta \in\left[0, \frac{1}{2}\right)$ and let $c \in \mathfrak{C}^{\delta}$. The gradient of $\mathfrak{U}^{\delta}$ at $c, \nabla \mathfrak{A}_{c}^{\delta}$ is the linear functional on $\Gamma_{0}(Q)$ which sends $\psi=(a, \phi)$ to

$$
\begin{aligned}
\nabla^{2} \mathfrak{U}_{c}^{\delta}(\psi)= & \left.\frac{d^{2}}{d t^{2}} \mathfrak{U}^{\delta}(c+t \psi)\right|_{t=0}=\left\langle\left(1+|x|^{2}\right)^{\delta} D_{A} a, F_{A}\right\rangle_{2} \\
& +\left\langle[a, \Phi], D_{A} \Phi\right\rangle_{2}+\left\langle D_{A} \phi, D_{A} \Phi\right\rangle_{2} .
\end{aligned}
$$

The hessian of $\mathfrak{U}^{\delta}$ at $c, \nabla^{2} \mathfrak{U}_{c}^{\delta}$ is the quadratic functional on $\Gamma_{0}(Q)$ which sends $\psi=(a, \phi)$ to

$$
\begin{aligned}
\nabla^{2} \mathfrak{U}_{c}^{\delta}(\psi)= & \left.\frac{d^{2}}{d t^{2}} \mathfrak{U}^{\delta}(c+t \psi)\right|_{t=0}=\left\langle\left(1+|x|^{2}\right)^{\delta} D_{A} a, D_{A} a\right\rangle_{2} \\
& +\left\langle\left(1+|x|^{2}\right)^{\delta}[a, a], F_{A}\right\rangle_{2}+\langle[a, \Phi],[a, \Phi]\rangle_{2} \\
& +2\left\langle[a, \phi], D_{A} \Phi\right\rangle_{2}+2\left\langle[a, \Phi], D_{A} \phi\right\rangle_{2}+\left\langle D_{A} \phi, D_{A} \phi\right\rangle_{2}
\end{aligned}
$$

In order to measure the size of $\nabla \mathfrak{A}_{c}^{\delta}$ and $\nabla^{2} \mathfrak{O}_{c}^{\delta}$, it is convenient to introduce new norms on $\Gamma_{0}(Q)$.

Definition $A .4 .2$. Let $\delta \in\left[0, \frac{1}{2}\right)$ and $c \in \mathfrak{C}^{\delta}$. Define the norm $\|\cdot\|_{c, \delta}$ on $\Gamma_{0}(Q)$ by assigning to $\psi=(a, \phi)$ the number

$$
\|\psi\|_{c, \delta}^{2}=\left\langle\left(1+|x|^{2}\right)^{\delta} \nabla_{A} a, \nabla_{A} a\right\rangle_{2}+\left\langle\nabla_{A} \phi, \nabla_{A} \phi\right\rangle_{2}+\langle[\Phi, \psi],[\Phi, \psi]\rangle_{2} .
$$

This norm was chosen because it allows one to establish the following uniform estimates.

Proposition A.4.3. Let $\delta \in\left[0, \frac{1}{2}\right)$. There exists a continuous, increasing function $z(\cdot):[0, \infty) \rightarrow[0, \infty)$ with the following property. For $c \in \mathfrak{C}^{\delta}$ let $z_{c}=z\left(\mathfrak{A}^{\delta}(c)\right)$, and for notational convenience, let $\|\cdot\|$ denote $\|\cdot\|_{c, \delta}$. For any $\psi, \eta \in \Gamma_{0}(Q)$,

(1) $\left|\mathfrak{P}^{\delta}(c+\psi)-\mathfrak{A}^{\delta}(c)\right| \leqq z_{c}\|\psi\|(1+\|\psi\|)^{3}$,

(2) $\left|\mathfrak{U}^{\delta}(c+\psi)-\mathfrak{U}^{\delta}(c)-\nabla \mathfrak{U}_{c}^{\delta}(\psi)\right| \leqq z_{c}\|\psi\|^{2}(1+\|\psi\|)^{2}$,

(3) $\left|\mathfrak{U}^{\delta}(c+\psi)-\mathfrak{U}^{\delta}(c)-\nabla \mathfrak{U}_{c}^{\delta}(\psi)-1 / 2 \nabla^{2} \mathfrak{U}_{c}^{\delta}(\psi)\right| \leqq z_{c}\|\psi\|^{3}(1+\|\psi\|)$,

(4) $\left|\nabla \mathfrak{U}_{c+\psi}^{\delta}(\eta)-\nabla \mathfrak{A}_{c}^{\delta}(\eta)\right| \leqq z_{c}\|\eta\|\|\psi\|(1+\|\psi\|)^{2}$,

(5) $\left|\nabla^{2} \mathfrak{A}_{c+\psi}^{\delta}(\eta)-\nabla^{2} \mathfrak{A}_{c}^{\delta}(\eta)\right| \leqq z_{c}\|\eta\|^{2}\|\psi\|(1+\|\psi\|)$.

Proof of Proposition A.4.3. This is an exercise in Hölder's inequality after using Lemmas A.4.1 and B.6.4 of [9]. The details are left to the reader. (But see Proposition 5.2 of $[6$, Part I $]$.)

By construction, $\nabla \mathfrak{U}_{c}^{\delta}$ satisfies the uniform estimate

$$
\left|\nabla \mathfrak{A}_{c}^{\delta}(\psi)\right| \leqq\|\psi\|_{c, \delta}\left(\mathfrak{U}^{\delta}(c)\right)^{1 / 2},
$$

while $\nabla^{2} \mathfrak{U}_{c}^{\delta}$ satisfies

$$
\left|\nabla^{2} \mathfrak{U}_{c}^{\delta}(\psi)\right| \leqq \zeta\left(\mathfrak{U}^{\delta}(c)\right)\|\psi\|_{c, \delta}^{2} .
$$

(Lemmas A.4.1 and B.6.4 of [9] must be used to derive Eq. (A.4.5).) A measure of the size of these two functionals is defined next. 
Definition A.4.4. Let $\delta \in\left[0, \frac{1}{2}\right)$ and $c \in \mathfrak{C}^{\delta}$. Define

$$
\left\|\nabla \mathfrak{A}_{c}^{\delta}\right\|_{*}=\sup _{0 \neq \psi \in \Gamma_{0}(Q)} \frac{\left|\nabla \mathfrak{I}_{c}^{\delta}(\psi)\right|}{\|\psi\|_{c, \delta}} .
$$

For each $l>0$, define

$$
\gamma_{c}^{\delta}(l)=\inf _{E \subset \Gamma_{0}(Q)}\left[\sup _{0 \neq \psi \in E} \frac{\nabla^{2} \mathfrak{I}_{c}^{\delta}(\psi)}{\|\psi\|_{c, \delta}^{2}}\right],
$$

where the infimum is over all $l$-dimensional linear subspaces $E$ in $\Gamma_{0}(Q)$.

A configuration $c \in \mathfrak{C}^{\delta}$ is a critical point of $\mathfrak{A}^{\delta}$ if and only if

$$
\left\|\nabla \mathfrak{U}_{c}^{\delta}\right\|_{*}=0 \text {. }
$$

The significance of $\gamma_{c}^{\delta}(l)$ is the following. This number is negative if and only if there exists an $l$-dimensional linear subspace of $\Gamma_{0}(Q)$ on which $\nabla^{2} \mathfrak{U}_{c}^{\delta}(\cdot)$ is negative.

The numbers $\left\|\nabla \mathfrak{U}_{c}^{\delta}\right\|_{*}$ and $\gamma_{c}^{\delta}(l)$ are $\mathfrak{G}$ invariant, so they can be considered as $S^{1}$ equivariant functions on $\hat{\mathfrak{B}}^{\delta}$.

Proposition A.4.5. Let $\delta \in\left[0, \frac{1}{2}\right)$, and let $l \in\{1,2, \ldots\}$. The assignment of $c \in \mathbb{C}^{\delta}$ to either $\left\|\nabla \mathfrak{U}_{c}^{\delta}\right\|_{*}$ or $\gamma_{c}^{\delta}(l)$ defines a continuous function on $\mathbb{C}^{\delta}$.

This section ends with the proof of Proposition A.4.5.

Proof of Proposition A.4.5. For $\delta=0$, Eq. (A.4.5) implies that $\nabla \mathfrak{A}_{(\cdot)}$ defines a continuous section over $\hat{\mathfrak{B}}$ of the vector bundle $H(Q)^{*}$ (see Sect. B.6 of [9]). Similarly, $\nabla^{2} \mathfrak{U}_{(\cdot)}$ defines a continuous section of $\operatorname{Sym}_{2} H(Q)^{*}$ over $\hat{\mathfrak{B}}$. As $\|\cdot\|_{(\cdot)}$ is a continuous fiber norm on $H(Q)$ over $\hat{\mathfrak{B}}$ (Proposition B.6.2 of [9]), the assertions in Proposition A.4.5 are automatically true.

For $\delta>0$, one can argue directly by adapting the arguments of Lemmas 6.2 and 6.3 of $[6$, Part I $]$ to the present situation. Only the case for $\nabla \mathfrak{A}_{(\cdot)}^{\delta}$ will be given. For notational convenience, for a sequence $\left\{c_{i}=\left(A_{i}, \Phi_{i}\right)\right\} \in \mathfrak{C}^{\delta}$, use $\nabla \mathfrak{A}_{i},\|\cdot\|_{i}$, for $\nabla \mathfrak{A}_{c}^{\delta}$, $\|\cdot\|_{c, \delta}$ and when $c=c_{i}$. Also use $\nabla_{i}, F_{i}$ for $\nabla_{A}$ and $F_{A}$ when $A=A_{i}$. As they are irrelevant, the $\delta$ sub and superscripts will be omitted.

Consider a sequence $\left\{c_{i}=\left(A_{i}, \Phi_{i}\right)\right\} \in \mathbb{C}$ which converges to $c=(A, \Phi) \in \mathbb{C}$. Given $\varepsilon>0$, there exists $\psi \in \Gamma_{0}(Q)$ such that $\|\psi\|_{c}=1$ and

$$
\nabla \mathfrak{U}_{c}(\psi)>\left\|\nabla \mathfrak{A}_{c}\right\|_{*}-\varepsilon .
$$

But $\psi$ has compact support, so $\lim \|\psi\|_{i}=1$ and $\lim \nabla \mathfrak{A}_{i}(\psi)=\nabla \mathfrak{A}_{c}(\psi)$. Therefore, $\lim \left\|\nabla \mathfrak{U}_{i}\right\|_{*} \geqq\left\|\nabla \mathfrak{U}_{c}\right\|_{*}$. Conversely, given $\varepsilon>0$, there exists for each $i, \psi_{i} \in \Gamma_{0}(Q)$ with $\left\|\psi_{i}\right\|_{i}=1$ and $\nabla \mathfrak{U}_{i}\left(\psi_{i}\right)>\left\|\nabla \mathfrak{U}_{i}\right\|_{*}-\varepsilon$. As $\left\{\left(A_{i}, \Phi_{i}\right)\right\}$ converges in $C^{\infty}$ on bounded domains, a diagonalization argument (as in [23]) shows that $\left\{\psi_{i}\right\}$ has a weakly convergent subsequence in $L_{1 ; \text { loc }}^{2}$ (cf. Sect. A.3.4 of [9]) with a limit, $\psi=(a, \phi) \in L_{1 ; \text { loc }}^{2}$. For this subsequence, write $\psi_{i}=\left(a_{i}, \phi_{i}\right)$. Then $y_{i}=\left((1+|x|)^{\delta} \nabla_{i} a_{i}\right.$, $\left.\nabla_{i} \phi_{i},\left[\Phi_{i}, \psi_{i}\right]\right)$ converges weakly in $L^{2}$ to some $y \in L^{2}$. As $y$ must equal $y_{c} \equiv\left((1+|x|)^{\delta} \nabla_{A} a, \nabla_{A} \phi,[\Phi, \psi]\right)$ on bounded domains of $\mathbb{R}^{3}$, one infers that $y \equiv y_{c}$ and therefore that $y_{c} \in L^{2}$. Further, $(1+|x|)^{\delta}\left|\psi_{i}\right| \in L^{6}$ is uniformly bounded (cf. Lemma A.4.1 of [9]) and so a similar argument shows that $(1+|x|)^{\delta}|\psi| \in L^{6}\left(\mathbb{R}^{3}\right)$. To 
summarize, $\left\{\psi_{i}\right\}$ has a weak limit, $\psi$, with $(1+|x|)^{\delta} \psi \in L_{1 ; \text { loc }}^{2} \cap L^{6}(Q)$ and $\|\psi\|_{c, \delta}=\|y\|_{2} \leqq \lim \left\|y_{i}\right\|_{2}=1$.

Define the Banach space $H_{c, \delta}(Q)$ as the completion of $\Gamma_{0}(Q)$ with the norm $\|\cdot\|_{c, \delta}$. Using mollifiers and Lemma A.4.1 of [9], one can readily show that $\psi \in H_{c, \delta}(Q)$.

Equation (A.4.5) implies that $\nabla \mathfrak{H}_{c}^{\delta}$ defines a bounded, linear functional on $H_{c, \delta}(Q)$ whose norm is just $\left\|\nabla \mathfrak{U}_{c}^{\delta}\right\|_{*}$.

Due to Proposition B.4.1 of $[9],\left\{\left((1+|x|)^{\delta} F_{i}, \nabla_{i} \Phi_{i}\right)\right\}$ converges strongly in $L^{2}$ to $\left.\left\{(1+|x|)^{\delta} F_{A}, \nabla_{A} \Phi\right)\right\}$. Therefore, weak $L^{2}$ convergence of $y_{i}$ to $y_{c}$ implies that $\lim \nabla \mathfrak{U}_{i}\left(\psi_{i}\right)=\nabla \mathfrak{A}_{c}^{\delta}(\psi)$. As $\|\psi\|_{c, \delta} \leqq 1$, one concludes that

$$
\left\|\nabla \mathfrak{U}_{c}^{\delta}\right\|_{*} \geqq \frac{1}{\|\psi\|_{c, \delta}} \nabla \mathfrak{U}_{c}^{\delta}(\psi) \geqq \lim \left\|\nabla \mathfrak{U}_{i}\right\|_{*}-\varepsilon .
$$

Therefore, $\left\|\nabla \mathfrak{U}_{(\cdot)}^{\delta}\right\|_{*}$ is continuous.

\section{B.1. The Regularized Problem}

Let $\mathfrak{F}$ be a homotopy invariant family of subsets of $\mathfrak{B}$. The regularized problem is to use $\mathfrak{F}$ to find a critical point of $\mathfrak{\mathfrak { U }}^{\delta}$ on $\hat{\mathfrak{B}}^{\delta}$. For this purpose, define

$$
\mathfrak{F}^{\delta}=\left\{F \in \mathfrak{F}: F \subset \hat{\mathfrak{B}}^{\delta}\right\}
$$

A crucial observation is

Lemma B.1.1. Let $\mathfrak{F}$ be as described above. Then for each $\delta \in\left(0, \frac{1}{2}\right), \mathfrak{F}^{\delta}$ as defined by Eq. (B.1.1) is not empty.

The proof of this lemma, and of Lemma B.1.2 below, are deferred to the end of this section.

To each homotopy invariant family $\mathfrak{F}$ and to each $\delta \in\left(0, \frac{1}{2}\right)$, assign the number

$$
\mathfrak{U}_{\mathfrak{F}}^{\delta}=\inf _{F \in \mathfrak{F}^{\delta}} \max _{c \in F} \mathfrak{U}^{\delta}(c) \text {. }
$$

Concerning $\mathfrak{U}_{\mathfrak{F}}^{\delta}$, one observes that $\mathfrak{U}_{\mathfrak{F}}^{\delta} \geqq \mathfrak{U}_{\mathfrak{F}}$ and

Lemma B.1.2. As a function of $\delta \in\left[0, \frac{1}{2}\right), \mathfrak{A}_{\mathfrak{F}}^{\delta}$ is non-decreasing; and $\lim _{\delta \rightarrow 0} \mathfrak{A}_{\mathfrak{F}}^{\delta}=\mathfrak{A}_{\mathfrak{F}}$.

The significance of $\mathfrak{P}_{\mathfrak{F}}^{\delta}$ is provided by Proposition B.1.3 below. The statement of the proposition refers to the family

$$
Y^{\delta}(\mathfrak{F})=\left\{(F, c) \in \mathfrak{F}^{\delta} \times \hat{\mathfrak{B}}^{\delta}: c \in F \quad \text { and } \quad \mathfrak{U}^{\delta}(c)=\sup _{F} \mathfrak{U}^{\delta}\right\}
$$

Proposition B.1.3. Let $\mathfrak{F}$ be a homotopy invariant family of compact subsets of $\mathfrak{\mathfrak { B }}$. Then for each $\delta \in\left(0, \frac{1}{2}\right)$, there exists a sequence $\left\{\left(F_{i}, c_{i}\right)\right\} \subset Y^{\delta}(\mathfrak{F})$ with the property that the sequence $\left\{c_{i}\right\}$ converges in $\hat{\mathfrak{B}}^{\delta}$ (as defined in Sect. A.3) to $c^{\delta}(\mathfrak{F})$, and this $c^{\delta}(\mathfrak{F})$ is a critical point of $\mathfrak{U}^{\delta}$ with critical value $\mathfrak{U}_{\mathfrak{\mho}}^{\delta}$.

The proof of Proposition B.1.3 occupies Sects. B.2-B.4. The proof requires the following technical lemmas. 
Lemma B.1.4. Let $\Psi:[0,1] \times \hat{\mathfrak{B}} \rightarrow \mathfrak{C}$ be a continuous map. Suppose that $\Psi(0, \cdot)$ $=$ identity. There exists a continuous map $h:[0,1] \times \hat{\mathfrak{B}} \rightarrow(\mathfrak{G}$ such that $h(0, \cdot)=1$ and $(h \Psi)(\cdot)$ maps $[0,1] \times \hat{\mathfrak{B}}$ continuously into $\hat{\mathfrak{B}}$.

Lemma B.1.5. Let $F \subset \hat{\mathfrak{B}}$ be a compact set. For some $\delta \in\left[0, \frac{1}{2}\right)$, suppose that $F \subset \hat{\mathfrak{B}}^{\delta}$. Given a neighborhood, $V$, of $F$ in $\hat{\mathfrak{B}}$, and $\varepsilon>0$, there exists a homotopy $\tau:[0,1] \times \hat{\mathfrak{B}} \rightarrow \hat{\mathfrak{B}}$ such that $\tau(1, \cdot)$ maps $\hat{\mathfrak{B}}$ continuously into $\hat{\mathfrak{B}}^{\delta}$, for all $t \in[0,1]$, $\tau(t, F) \subset V$ and for all $b \in F, \mathfrak{Q}^{\delta}(\tau(1, b))<\varepsilon+\sup _{F} \mathfrak{H}^{\delta}$.

The proofs of Lemmas B.1.1-2 and B.1.4-5 complete this section.

Proof of Lemma B.1.1. Proposition B.5.2 of [9] provides a continuous map $q:[0,1] \times[0,1] \times \hat{\mathfrak{B}} \rightarrow \mathfrak{\mathcal { B }}$ with the property that for each $\varepsilon \in(0,1], q(\varepsilon, \cdot, \cdot):[0,1]$ $\times \hat{\mathfrak{B}} \rightarrow \mathfrak{\mathcal { B }}$ satisfies $q(\varepsilon, 0, \cdot)=$ identity and $q(\varepsilon, 1, \cdot)$ maps $\hat{\mathfrak{B}}$ continuously into $\hat{\mathfrak{B}}^{\delta}$ for each $\delta \in\left[0, \frac{1}{2}\right)$. If $F \in \mathfrak{F}$, then as long as $\varepsilon \in(0,1), q^{\varepsilon}(1, F)=q(\varepsilon, 1, F) \in \mathfrak{F}^{\delta}$.

Proof of Lemma B.1.2. The assertion that $\mathfrak{A}_{\mathfrak{F}}^{\delta} \geqq \mathfrak{H}_{\mathfrak{F}}^{\gamma}$ when $\frac{1}{2}>\delta>\gamma \geqq 0$ follows from the fact that for any $c \in \mathfrak{C}^{\delta}, \mathfrak{I}^{\delta}(c) \geqq \mathfrak{A}^{\gamma}(c)$ for $\delta \geqq \gamma$. To prove the second assertion, choose $\varepsilon>0$ and $F \in \mathfrak{F}$ with $\max _{F} \mathfrak{A} \leqq \mathfrak{A}_{\mathfrak{F}}+\frac{1}{3} \varepsilon$. According to Proposition B.5.2 of [9], $F(\varepsilon)=q^{\varepsilon / 3}(1, F)$ has the property that $F(\varepsilon) \subset \bigcap_{\delta} \hat{\mathfrak{B}}^{\delta}$ and the max of $\mathfrak{A}$ on $F(\varepsilon)$ is less than $\mathfrak{A}_{\mathfrak{F}}+\frac{2}{3} \varepsilon$. The fact that $F(\varepsilon) \subset \bigcap_{\delta} \mathfrak{B}^{\delta}$ implies that $\mathfrak{U}^{\delta}$ is continuous on $F(\varepsilon)$ for all $\delta \in\left[0, \frac{1}{2}\right)$. Now, for any fixed $b \in \mathfrak{B}^{\delta}$,

$$
\lim _{\delta \rightarrow 0} \mathfrak{U}^{\delta}(b)=\mathfrak{A}(b),
$$

and this limit is uniform on compact subsets of $\bigcap_{\delta} \hat{\mathfrak{B}}^{\delta}$. Thus, $\delta(\varepsilon)>0$ exists such that for all $\delta \in[0, \delta(\varepsilon))$, and for all $b \in F(\varepsilon), \mathfrak{a}^{\delta}(b)<\mathfrak{A}_{\widetilde{\mho}}+\varepsilon$. For such $\delta$, $\mathfrak{U}_{\mathfrak{\mho}}^{\delta}<\mathfrak{A}_{\mathfrak{F}}+\varepsilon$, too.

Proof of Lemma B.1.4. The projection map, $\mathfrak{C} \rightarrow \mathfrak{B}$ (see B.1 of [9]) produces a continuous $g:[0,1] \times \hat{\mathfrak{B}} \rightarrow\left(\mathfrak{G}_{0}\right.$ such that $(g \cdot \Psi):[0,1] \times \hat{\mathfrak{B}} \rightarrow \mathfrak{B}$. Section B.5 of $[9]$ defines a $\mathfrak{G}$ equivariant map $\hat{n}: \mathfrak{B} \rightarrow S^{2}$ with the property that $\hat{\mathfrak{B}}=\hat{n}^{-1}$ (north pole) (Eq. (B.5.2) of [9]). The homotopy lifting property of the fibration $S^{1} \rightarrow \mathrm{SU}(2) \stackrel{\pi}{\rightarrow} S^{2}$ provides a continuous $l:[0,1] \times \hat{B} \rightarrow \mathrm{SU}(2)$ with the property that $l(0, \cdot)=1 \in \mathrm{SU}(2)$ and

$$
\pi \cdot l=\hat{n}(g \cdot \Psi) .
$$

By using the embedding of SU(2) in $\mathfrak{G}\left(=C^{\infty}\left(\mathbb{R}^{3} ; \mathrm{SU}(2)\right)\right.$ as the constant maps, one obtains $l^{-1} g:[0,1] \times \mathfrak{B} \rightarrow\left(\mathfrak{G}\right.$. Equation $(B .1 .3)$ implies that $\left(l^{-1} g \cdot \Psi\right)(\cdot):[0,1]$ $\times \hat{\mathfrak{B}} \rightarrow \mathfrak{B}$, and $\left(l^{-1} g \cdot \Psi\right)(0, \cdot)=$ identity.

Proof of Lemma B.1.5. Given $F$ and $V$, it follows from Proposition B.5.2 of [9] that there exists $\lambda_{0} \in(0,1)$ such that for all $0<\lambda<\lambda_{0}$, the homotopy $q(\lambda, \cdot, \cdot):[0,1]$ $\times \hat{\mathfrak{B}} \rightarrow \mathfrak{B}$ satisfies $q(\lambda, t, F) \subset V$ for all $t \in[0,1]$ and also $q(\lambda, 1, \cdot)$ maps $\mathfrak{B}$ continuously into $\hat{\mathfrak{B}}^{\delta}$. As $q(\cdot, \cdot, \cdot)$ restricts to a continuous map from $[0,1]$ $\times[0,1] \times \hat{\mathfrak{B}}^{\delta}$ to $\hat{\mathfrak{B}}^{\delta}$, for each $b \in F$, there exists $\lambda(b)>0$ such that for all $0<\lambda<\lambda(b)$, $\mathfrak{A}^{\delta}(q(\lambda, 1, b))<\sup _{F} \mathfrak{H}^{\delta}+\varepsilon$. But $\mathfrak{H}^{\delta}$ is continuous on $\hat{\mathfrak{B}}^{\delta}$ so there is a neighborhood, 
$N_{b} \subset \hat{\mathfrak{B}}^{\delta}$ of $q(\lambda, 1, b)$ such that for all $b^{\prime} \in N_{b}, \mathfrak{U}^{\delta}\left(b^{\prime}\right)<\sup _{F} \mathfrak{I}^{\delta}+\varepsilon$. As $\bigcup_{b \in F} q^{-1}(\lambda(b)$, $1, \cdot)\left(N_{b} \cap F\right)$ is an open cover of $F$ and $F$ is compact, this cover has a finite subcover. As a consequence, there exists $\lambda(F, \varepsilon)>0$ such that for any $0<\lambda<\lambda(F, \varepsilon)$, $\tau=q(\lambda, \cdot, \cdot)$ satisfies Lemma B.1.5's requirements.

\section{B.2. Minimizing Sets for $\mathfrak{A}^{\boldsymbol{\delta}}$}

Let $\mathfrak{F}$ be a homotopy invariant family of compact subsets of $\mathfrak{B}$. The purpose of this section is to construct min-max sequences $\left\{\left(F_{i}, c_{i}\right)\right\}$ in $Y^{\delta}(\mathfrak{F})$ with special properties which will facilitate the proof of Proposition B.1.3. The goal is to use appropriately chosen, "pseudo-gradient" homotopies of $\mathfrak{\mathfrak { B }}$ to construct such $\left\{\left(F_{i}, c_{i}\right)\right\}$ for which each $c_{i}$ satisfies a priori estimates which make it look like a solution to the variational equation of $\mathfrak{U}^{\delta}$.

Proposition B.2.1. Let $\mathfrak{F}$ be as described above. Let $\delta \in\left[0, \frac{1}{2}\right)$. Given any $\varepsilon>0$, there exists $(F, c) \in Y^{\delta}(\mathfrak{F})$ with the following properties:

(1) $\mathfrak{U}^{\delta}(c)<\mathfrak{U}^{\delta}(\mathfrak{F})+\varepsilon$

(2) $\left\|\nabla \mathfrak{U}_{c}^{\delta}\right\|_{*}<\varepsilon$,

(3) Every $b=(A, \Phi) \in F$ satisfies $\nabla_{A}^{*} \nabla_{A} \Phi=0$,

(4) Every $b=(A, \Phi) \in F$ satisfies

$$
\left\langle\left(1+|\cdot|^{2}\right)^{\delta} F_{A}, F_{A}\right\rangle_{2}=(1-2 \delta)^{-1}\left(\left\langle\nabla_{A} \Phi, \nabla_{A} \Phi\right\rangle_{2}-2 \delta\left\langle\left(1+|\cdot|^{2}\right)^{-1+\delta} F_{A}, F_{A}\right\rangle_{2}\right. \text {. }
$$

The proposition is proved in four steps. The first produces a homotopy of $\mathfrak{\mathfrak { B }}$ which deforms a given $G \in \mathfrak{F}^{\delta}$ to $G_{1} \in \mathfrak{F}^{\delta}$ satisfying Assertion (3). A second homotopy of $\hat{\mathfrak{B}}$ is then constructed which preserves the condition expressed in Assertion (3) and deforms $G_{1}$ to $F^{\prime} \in \mathfrak{F}^{\delta}$ which satisfies Assertions (3) and (4). Both these homotopies decrease $\mathfrak{U}^{\delta}$. For the third step, a $G \in \mathfrak{F}^{\delta}$ is chosen to satisfy $\max _{G} \mathfrak{A}^{\delta}<\mathfrak{U}_{\mathfrak{F}}^{\delta}+\mu \varepsilon$ for a suitable $\mu \in(0,1)$. The resulting $F^{\prime}$ is deformed by a pseudo-gradient vector field for $\mathfrak{U}^{\delta}$ to $F^{\prime \prime} \in \mathfrak{F}^{\delta}$ so that at the points in $F^{\prime \prime}$ where $\mathfrak{U}^{\delta}$ is maximized, Assertions (1) $(4)$ are satisfied. Then the first two homotopies, above, are reapplied to obtain $F \in \mathfrak{F}^{\delta}$ whose points where $\mathfrak{I}^{\delta}$ is maximal are in the same (5) orbit as those in $F^{\prime \prime}$ where $\mathfrak{U}^{\delta}$ is maximal. For this $F$, Assertions (1)-(4) are satisfied. A similar technique was used to prove Proposition 5.2 of [6, Part I]. See also Sect. 5 of [22].

The remainder of this section contains the proof of Proposition B.2.1. The first step is to use Proposition B.3.2 of [9]. It provides a continuous map $c_{0}: \hat{\mathfrak{B}}^{\delta} \rightarrow \hat{\mathfrak{B}}^{\delta}$ for each $\delta \in\left[0, \frac{1}{2}\right)$ which sends $c=(A, \Phi)$ to $c_{0}(c)=\left(A, \Phi_{0}(c)\right)$, where $\nabla_{A}^{*} \nabla_{A} \Phi_{0}(c)=0$.

Lemma B.2.2. Given $\mathfrak{F}$ and $\delta$ as in Proposition B.2.1, and given $\varepsilon>0$ and $G \in \mathfrak{F}^{\delta}$, there exists a homotopy $\tau_{1}:[0,1] \times \hat{\mathfrak{B}} \rightarrow \mathfrak{B}$ such that $F=\tau_{1}(1, G) \in \mathfrak{F}^{\delta}$ has the properties:

(1) $\max _{F} \mathfrak{A}^{\delta}<\max _{G} \mathfrak{U}^{\delta}+\varepsilon$.

(2) F satisfies Assertion (3) of Proposition B.2.1. 
Proof of Lemma B.2.2. Choose an open $V \subset \mathfrak{B}$ that contains $G$ and construct $\tau:[0,1] \times \hat{\mathfrak{B}} \rightarrow \mathfrak{B}$ for $G, V$, and $\varepsilon$ as specified by Lemma B.1.5. Let $F_{1}=\tau(1, G)$. Then $F_{1}$ satisfies Assertion (1) of Lemma B.2.2. Now consider the map $\hat{c}:[0,1] \times \hat{\mathfrak{B}} \rightarrow \mathfrak{B}$ which sends $(t, c)$ to

$$
\hat{c}(t, c)=c+t\left(c_{0}(c)-c\right) .
$$

Proposition B.3.2 of [9] insures that $\hat{c}$ is continuous and that it maps $[0,1] \times \hat{\mathfrak{B}}^{\delta} \rightarrow \mathfrak{B}^{\delta} \quad$ continuously. For fixed $c \in \hat{\mathfrak{B}}^{\delta}, \quad \mathfrak{U}^{\delta}\left(\hat{c}_{0}(\cdot, c)\right)$ and also $\mathfrak{U}\left(\hat{c}_{0}(\cdot, c)\right)$ are non-increasing on $[0,1]$ due to their convexity in $\Phi$. Let $\tau^{\prime}:[0,1] \times \hat{\mathfrak{B}} \rightarrow \mathfrak{B}$ be defined so to send $(t, b)$ to

$$
\tau^{\prime}(t, b)= \begin{cases}\tau(2 t, b) & \text { for } t \in\left[0, \frac{1}{2}\right], \\ \hat{c}(2 t-1, \tau(1, b)) & \text { for } t \in\left[\frac{1}{2}, 1\right] .\end{cases}
$$

Lemma B.1.4 provides a continuous $h:[0,1] \times \hat{\mathfrak{B}} \rightarrow \mathfrak{G}$ such that $\tau_{1}=h \tau^{\prime}$ is a continuous homotopy of $\hat{\mathfrak{B}}^{\delta}$ for every $\delta \in\left[0, \frac{1}{2}\right)$. This $\tau_{1}$ satisfies the assertions of Lemma B.2.2.

Lemma B.2.3. Given $\mathfrak{F}$ and $\delta$ as in Proposition B.2.1, and given $\varepsilon>0$ and $G \in \mathfrak{F}^{\delta}$, there exists a homotopy $\tau_{2}:[0,1] \times \hat{\mathfrak{B}} \rightarrow \mathfrak{B}$ such that $F=\tau_{2}(1, G) \in \mathfrak{F}^{\delta}$ has the properties

(1) $\max _{F} \mathfrak{A}^{\delta}<\max _{G} \mathfrak{A}^{\delta}+\varepsilon$, and

(2) F satisfies Assertions (3) and (4) of Proposition B.2.1.

The proof of Lemma B.2.3 is delayed as it requires the construction of a specific homotopy of $\hat{\mathfrak{B}}$. To begin, let $\delta \in\left[0, \frac{1}{2}\right)$ and let $\lambda: \hat{\mathfrak{B}}^{\delta} \rightarrow(0, \infty)$ be a continuous function. Then $\lambda$ induces a continuous function, $\hat{\lambda}: \hat{\mathfrak{B}}^{\delta} \rightarrow C^{\infty}\left(\mathbb{R}^{3} ; \mathbb{R}^{3}\right)$ which sends $c \in \hat{\mathfrak{B}}^{\delta}$ and $x \in \mathbb{R}^{3}$ to $\hat{\lambda}(c)(x)=\lambda(c) \cdot x$. Thus, any $\lambda: \hat{\mathfrak{B}}^{\delta} \rightarrow(0, \infty)$ defines a map, denoted $\lambda^{*}$, from $\hat{\mathfrak{B}}^{\delta}$ to $\mathfrak{B}^{\delta}$ which sends $c=(A, \Phi)$ to the pulled back configuration

$$
\lambda^{*}(c)=\left(\hat{\lambda}^{*}(c) A, \hat{\lambda}^{*}(c) \Phi\right),
$$

where $\left(\hat{\lambda}^{*} \Phi\right)(x)=\Phi(\lambda x)$ and $\left(\hat{\lambda}^{*} A\right)(x)=\lambda A(\lambda x)$.

Lemma B.2.4. Let $\lambda:[0,1] \times \hat{\mathfrak{B}}^{\delta} \rightarrow(0, \infty)$ be continuous. For each $t \in[0,1]$, define $\lambda^{*}(t, \cdot): \hat{\mathfrak{B}}^{\delta} \rightarrow \mathfrak{B}^{\delta}$ as above. Then the assignment of $(t, c) \in[0,1] \times \hat{\mathfrak{B}}^{\delta}$ to $\lambda^{*}(t, c) \in \mathfrak{B}^{\delta}$ is continuous.

Proof of Lemma B.2.4. The fixed product structure on $P=\mathbb{R}^{3} \times \mathrm{SU}(2)$ identifies $\hat{\mathfrak{B}}^{\delta}$ as a subset of $\underset{12}{\times} C^{\infty}\left(\mathbb{R}^{3}\right)$ (Sect. B.5 of [9]). The map $\lambda^{*}(\cdot, \cdot)$ is continuous with respect to the induced topology. The reader can check this using the definitions in Sects. A.3, B.1-2 of [9]. It remains to show that the composition, $\mathfrak{\mathfrak { A }}^{\delta}\left(\lambda^{*}(\cdot, \cdot)\right)$ : $[0,1] \times \hat{\mathfrak{B}}^{\delta} \rightarrow[0, \infty)$ is continuous. However, $\mathfrak{A}^{\delta}\left(\lambda^{*}(\cdot, \cdot)\right)$ is a composition. First define $l: \hat{\mathfrak{B}}^{\delta} \rightarrow C^{\infty}((0, \infty))$ by sending $c \in \hat{\mathfrak{B}}^{\delta}$ and $t \in(0, \infty)$ to

$$
l(c, t)=t\left\langle\left(1+t^{-2}|\cdot|^{2}\right)^{\delta} F_{A}, F_{A}\right\rangle_{2}+t^{-1}\left\langle D_{A} \Phi, D_{A} \Phi\right\rangle_{2} .
$$

The map $l$ is evidently continuous, and by viewing $l$ as a continuous map from $\hat{\mathfrak{B}}^{\delta} \times[0, \infty) \rightarrow(0, \infty)$, one observes that $\mathfrak{U}^{\delta}\left(\lambda^{*}(\cdot, \cdot)\right)$ is the continuous map $l(\cdot, \lambda(\cdot, \cdot))$. 
Now, for each $\delta \in\left[0, \frac{1}{2}\right)$, let $\mathfrak{P}^{\delta}=\left\{(A, \Phi) \in \mathfrak{\mathfrak { B }}^{\delta}: F_{A} \neq 0\right.$ and $\left.D_{A} \Phi \neq 0\right\}$. This $\mathfrak{P}^{\delta}$ is an open, dense set in $\mathfrak{\mathfrak { B }}^{\delta}$.

Lemma B.2.5. For fixed $c \in \mathfrak{B}^{\delta}$, the $C^{\infty}$ function $l(c, \cdot):(0, \infty) \rightarrow(0, \infty)$ given by $E q$. (B.2.4) has exactly one critical point, its minimum $s(c)$. The assignment of $c$ to $s(c)$ defines a continuous map from $\mathfrak{P}^{\delta}$ to $(0, \infty)$.

Proof of Lemma B.2.5. First, if $c \in \mathfrak{P}^{\delta}$, then $l(c, t)$ goes to $\infty$ as $t \rightarrow 0$ or $\infty$ and so $l(c, \cdot)$ has at least one critical point on $(0, \infty)$. If $s$ is a critical point of $l(c, \cdot)$, then

$$
\begin{aligned}
0= & \left.\frac{d}{d t} l(c, \cdot)\right|_{s}=(1-2 \delta)\left\langle\left(1+s^{-2}|\cdot|^{2}\right)^{\delta} F_{A}, F_{A}\right\rangle_{2} \\
& +2 \delta\left\langle\left(1+s^{-2}|\cdot|^{2}\right)^{-1+\delta} F_{A}, F_{A}\right\rangle_{2}-s^{-2}\left\langle D_{A} \Phi, D_{A} \Phi\right\rangle_{2}
\end{aligned}
$$

By a direct calculation using Eq. (B.2.5), one can check that

$$
\left.\frac{d^{2}}{d t^{2}} l(c, \cdot)\right|_{s}>0 \text {. }
$$

Thus, $l(c, \cdot)$ has only minima, and hence just one. Equation (B.2.6) and the implicit function theorem imply that the unique minimum, $s(c)$, is a continuous function of $c$.

These last two lemmas are applied in

Lemma B.2.6. Given an open set $V \subset \hat{\mathfrak{B}}^{\delta}$ such that $\hat{\mathfrak{B}}^{\delta} \backslash \mathfrak{P}^{\delta} \subset V$, there exists a continuous map $m:[0,1] \times \hat{\mathfrak{B}}^{\delta} \rightarrow \mathfrak{B}^{\delta}$ with the following properties: (1) $m(0, \cdot)=$ identity; (2) $m$ fixes $\hat{\mathfrak{B}}^{\delta} \backslash \mathfrak{P}^{\delta}$; (3) For all $c \in \hat{\mathfrak{B}}^{\delta}, \mathfrak{A}^{\delta}(m(\cdot, c))$ is nonincreasing; and (4) for $c \in \hat{\mathfrak{B}}^{\delta} \backslash V$, $m(1, c)=s^{*}(c)$, where $s^{*}(\cdot)$ is constructed from $s(\cdot)$ of Lemma B.2.5 with Eq. (B.2.3). Thus, for such $c, m(1, c)$ satisfies Eq. (B.2.1).

Proof of Lemma B.2.6. As $\hat{\mathfrak{B}}^{\delta}$ is paracompact, there is a $\varrho \in C^{0}\left(\hat{\mathfrak{B}}^{\delta} ;[0,1]\right)$ which is identically 1 on $\hat{\mathfrak{B}}^{\delta} \backslash V$ and identically 0 on $\hat{\mathfrak{B}}^{\delta} \backslash \mathfrak{B}^{\delta}$. Consider the map from [0,1] $\times \hat{\mathfrak{B}}^{\delta}$ to $(0, \infty)$ which sends $(t, c)$ to $\lambda(t, c)=1+\varrho(c) t(s(c)-1)$, where Lemma B.2.5 defines $s(c)$. This $\lambda(\cdot, \cdot)$ is continuous on its domain of definition. For $c \in \mathfrak{B}^{\delta} \backslash V$, $\lambda(1, c)=s(c)$, and for $c \in \mathfrak{B}^{\delta} \backslash \mathfrak{P}^{\delta}, \lambda(t, c)=1$ for all $t \in[0,1]$. Define $m$ to send $(t, c) \in[0,1] \times \hat{\mathfrak{B}}^{\delta}$ to $\lambda^{*}(t, c) \in \hat{\mathfrak{B}}^{\delta}$ where $\lambda^{*}(\cdot, \cdot)$ is given by Lemma B.2.4.

Proof of Lemma B.2.3. Given $\varepsilon>0$, one can construct readily a homotopy, $\tau_{0}:[0,1] \times \hat{\mathfrak{B}} \rightarrow \mathfrak{\mathfrak { B }}$ such that $G^{\prime}=\tau_{0}(1, G)$ satisfies

$$
\begin{aligned}
\max _{G^{\prime}} \mathfrak{A}^{\delta}<\max _{G} \mathfrak{A}^{\delta}+\frac{\varepsilon}{2}, \\
G^{\prime} \cap \hat{\mathfrak{B}}^{\delta} \backslash \mathfrak{P}^{\delta}=\emptyset .
\end{aligned}
$$

One can require, if need be, that $G^{\prime}$ be arbitrarily close to $G$. Indeed, because $G$ is compact, one can find $a \in \Gamma_{0}\left(\operatorname{Ad} P \otimes T^{*}\right)$ of arbitrarily small $C^{k}, k \geqq 0$ and $L_{1}^{2}$ norms such that for all $c \in G, c+(a, 0) \in \mathfrak{P}^{\delta}$. Then for $(t, b) \in[0,1] \times \mathfrak{B}$, set $\tau_{0}(t, b)=b+t(a, 0)$. 
With $\left(G^{\prime}, \frac{\varepsilon}{2}\right)$ in place of $(G, \varepsilon)$ of Lemma B.2.2, construct the homotopy $\tau_{1}:[0,1] \times \hat{\mathfrak{B}} \rightarrow \mathfrak{\mathcal { B }}$. Let $F^{\prime}=\tau_{1}\left(1, G^{\prime}\right)$. Notice that $F^{\prime} \subset \mathfrak{B}^{\delta}$ and therefore, since $F^{\prime}$ is compact, there is an open set $V \subset \hat{\mathfrak{B}}^{\delta}$ such that $V \cap F^{\prime}=\emptyset$ and $\hat{\mathfrak{B}}^{\delta} \backslash \mathfrak{P}^{\delta} \subset V$. Use $V$ and Lemma B.2.6 to construct the map $m:[0,1] \times \hat{\mathfrak{B}}^{\delta} \rightarrow \mathfrak{B}^{\delta}$ of that lemma. Notice that for all $c \in \hat{\mathfrak{B}}^{\delta}$,

$$
\mathfrak{U}^{\delta}(m(1, c)) \leqq \mathfrak{H}^{\delta}(c)
$$

Finally, for $(t, b) \in[0,1] \times \hat{\mathfrak{B}}$, set

$$
\tau_{2}^{\prime}(t, b)=\left\{\begin{array}{lll}
\tau_{0}(3 t, b) & \text { if } & t \in\left[0, \frac{1}{3}\right] \\
\tau_{1}\left(3 t-1, \tau_{0}(1, b)\right) & \text { if } & t \in\left[\frac{1}{3}, \frac{2}{3}\right] \\
m\left(3 t-2, \tau_{1}(1, b)\right) & \text { if } & t \in\left[\frac{2}{3}, 1\right]
\end{array}\right.
$$

Then $\tau_{2}^{\prime}$ is a continuous map from $[0,1] \times \mathfrak{B}$ to $\mathfrak{B}$ with $\tau_{2}^{\prime}(0, \cdot)=$ identity. Lemma B.1.4 provides $h:[0,1] \times \hat{\mathfrak{B}} \rightarrow \mathfrak{G}$ such that $\tau_{2}=h \tau_{2}^{\prime}$ maps $[0,1] \times \hat{\mathfrak{B}} \rightarrow \widehat{\mathfrak{B}}$ continuously. Due to Eq. (B.2.8) and Lemma B.2.2, $\tau_{2}(1, G)$ satisfies Assertion (1) of Lemma B.2.3. Assertion (2) of that lemma is satisfied because for $c \in \mathfrak{B}^{\delta} \backslash V$, Lemma B.2.6 assures that $m(1, c)$ satisfies Eq. (B.2.1); and if $c=(A, \Phi)$ additionally satisfies $\nabla_{A}^{*} \nabla_{A} \Phi=0$, then $m(1, c)=\left(A^{\prime}, \Phi^{\prime}\right)$ satisfies $\nabla_{A^{\prime}}^{*} \nabla_{A^{\prime}} \Phi^{\prime}=0$.

Proof of Proposition B.2.1. Fix $\mu>0$. Given $\varepsilon$, choose $G \in \mathfrak{F}^{\delta}$ such that $\max _{G} \mathfrak{A}^{\delta}<\mathfrak{U}_{\mathfrak{F}}^{\delta}+\mu \varepsilon$. Using $G$ and $\mu \varepsilon$ in Lemma B.2.3, construct the homotopy $\tau_{2}$ and let $F^{\prime}=\tau_{2}(1, G)$. This $F^{\prime} \in \mathfrak{F}^{\delta}$ satisfies Assertions (3) and (4) of Proposition B.2.1 and it satisfies Assertion (1) with $2 \mu \varepsilon$ instead of $\varepsilon$. The goal now is to construct a homotopy of $\hat{\mathfrak{B}}, \tau_{4}$, which satisfies Assertions (1)-(4). For this purpose, define the sets

$$
\Omega_{0}=\left\{b \in \hat{\mathfrak{B}}^{\delta}: \mathfrak{U}^{\delta}(b)>\mathfrak{U}_{\mathfrak{Z}}^{\delta}\right\}
$$

and

$$
\Omega_{1}=\left\{b \in \Omega_{0}:\left\|\nabla \mathfrak{U}_{b}^{\delta}\right\|_{*}^{2}\left(1+\left\|\nabla \mathfrak{U}_{b}^{\delta}\right\|_{*}\right)^{-1}>4\left(1+8 z\left(\mathfrak{U}^{\delta}(b)\right)\right) \cdot\left(\mathfrak{U}^{\delta}(b)-\mathfrak{U}_{\mathfrak{\mho}}^{\delta}\right)\right\},
$$

where $z(\cdot):[0, \infty) \rightarrow[0, \infty)$ is the continuous function in Proposition A.4.3. Define also a number

$$
\mathfrak{I}_{*}^{\delta}=\max _{b \in \partial\left(\Omega_{1} \cap F^{\prime}\right)} \mathfrak{I}^{\delta}(b)
$$

Next, a pseudo-gradient "vector field" for $\mathfrak{U}^{\delta}$ is required. To construct one, note that at each $b \in \mathfrak{B}^{\delta}$, there exists a section $\psi_{b} \in \Gamma_{0}(Q)$ such that $\left\|\psi_{b}\right\|_{b}=\left\|\nabla \mathfrak{U}_{b}^{\delta}\right\|_{*}$, and $\nabla \mathfrak{U}_{b}^{\delta}\left(\psi_{b}\right) \leqq-\frac{1}{2}\left\|\nabla \mathfrak{U}_{b}^{\delta}\right\|_{*}$. As $\hat{\mathfrak{B}}^{\delta}$ is paracompact (Sects. B.1-B.2 of [9]), one can construct with $\left\{\psi_{b}, b \in \hat{\mathfrak{B}}^{\delta}\right\}$ and a locally finite partition of unity, a continuous $\psi: \hat{\mathfrak{B}}^{\delta} \rightarrow \Gamma_{0}(Q)$ with the property that at each $b \in \hat{\mathfrak{B}}^{\delta}$,

$$
\begin{aligned}
& \left|\|\psi(b)\|_{b}-\left\|\nabla \mathfrak{A}_{b}^{\delta}\right\|_{*}\right|<\mu \varepsilon, \text { and } \\
& \nabla \mathfrak{U}_{b}^{\delta}(\psi(b)) \leqq-\frac{1}{2}\left\|\nabla \mathfrak{A}_{b}^{\delta}\right\|_{*}+\mu \varepsilon .
\end{aligned}
$$


Let $\theta(\cdot)$ be the standard step function on $\mathbb{R} ; \theta(t)=1$ if $t>0$, and $\theta(t)=0$ if $t<0$. A map $v: \hat{\mathfrak{B}}^{\delta} \rightarrow \Gamma_{0}(Q)$ is defined as follows: In $\hat{\mathfrak{B}}^{\delta} \backslash \Omega_{1}$, set $v \equiv 0$ and in $\Omega_{1}$,

$$
v(b)=4\left\|\nabla \mathfrak{U}_{b}^{\delta}\right\|_{*}^{-2}\left(\mathfrak{U}^{\delta}(b)-\mathfrak{U}_{*}^{\delta}\right) \theta\left(\mathfrak{A}^{\delta}(b)-\mathfrak{U}_{*}^{\delta}\right) \psi(b) .
$$

Observe that $v$ is continuous and $\|v(b)\|_{b}<1$.

Define a continuous map $\hat{v}:[0,1] \times \mathfrak{B}^{\delta} \rightarrow \mathbb{C}^{\delta}$ by sending $(t, b)$ to

$$
\hat{v}(t, b)=b+t v(b) \text {. }
$$

When $b \notin \Omega_{1}$, then $\hat{v}(t, b)=b$ for all $t \in[0,1]$. But when $b \in \Omega_{1}$, one computes with Assertion (2) of Proposition A.4.3 that

$$
\mathfrak{U}^{\delta}(v(1, b)) \leqq \mathfrak{A}^{\delta}(b)-\left(\mathfrak{U}^{\delta}(b)-\mathfrak{A}_{*}\right) \theta\left(\mathfrak{A}^{\delta}(b)-\mathfrak{U}_{*}\right) \leqq \mathfrak{U}_{*} .
$$

Now, define a map $\tau_{3}^{\prime}:[0,1] \times \hat{\mathfrak{B}} \rightarrow \mathfrak{C}$ by sending $(t, b)$ to

$$
\tau_{3}^{\prime}(t, b)=\left\{\begin{array}{lll}
\tau_{2}(2 t, b) & \text { if } t \in\left[0, \frac{1}{2}\right], \\
v\left(2 t-1, \tau_{2}(1, b)\right) & \text { if } t \in\left[\frac{1}{2}, 1\right] .
\end{array}\right.
$$

This map $\tau_{3}^{\prime}$ is continuous, and $\tau_{3}(0, \cdot)=$ identity. Lemma B.1.4 provides a continuous $h:[0,1] \times \hat{\mathfrak{B}} \rightarrow\left(\mathfrak{G}\right.$ such that $\tau_{3}=h \tau_{3}^{\prime}$ maps $[0,1] \times \hat{\mathfrak{B}}$ continuously into $\hat{\mathfrak{B}}$ and actually with image in $\hat{\mathfrak{B}}^{\delta}$. Let $F^{\prime \prime}=\tau_{3}(1, G)$.

Lemma B.2.7. Define $\tau_{3}$ and $F^{\prime \prime}$ as above. Then

(1) $F^{\prime \prime} \in \mathfrak{F}^{\delta}$

If $c \in F^{\prime \prime}$ satisfies $\mathfrak{U}^{\delta}(c)=\max _{F^{\prime \prime}} \mathfrak{U}^{\delta}$, then

(2) $\mathfrak{A}^{\delta}(c)<\mathfrak{U}_{\mathfrak{F}}^{\delta}+2 \mu \varepsilon$,

(3) $\left\|\nabla \mathfrak{A}^{\delta}(c)\right\|_{*} \leqq \max \left[16\left(1+8 z\left(\mathfrak{U}^{\delta}(b)\right)\right) \mu \varepsilon,\left(16\left(1+8 z\left(\mathfrak{U}^{\delta}(b)\right)\right) \mu \varepsilon\right)^{1 / 2}\right]$,

(4) $c=(A, \Phi)$ satisfies $\nabla_{A}^{*} \nabla_{A} \Phi=0$ and Eq. (B.2.1).

Proof of Lemma B.2.7. Assertion (1) is true because $\tau_{3}(1, \cdot): \mathfrak{\mathfrak { B }} \rightarrow \hat{\mathfrak{B}}^{\delta}$ continuously. Assertion (2) is true because it is true for $F^{\prime}=\tau_{2}(1, G)$ and $\max _{F^{\prime \prime}} \mathfrak{A}^{\delta} \leqq \max _{F^{\prime}} \mathfrak{A}^{\delta}$. Assertion (3) is true because of Eq. (B.2.15). Every $c \in F^{\prime \prime}$ at which $\mathfrak{U}^{\delta}$ is maximal lies in the (5) orbit of $F^{\prime} \backslash\left(F^{\prime} \cap \Omega_{1}\right)$. For this same reason, Assertion (4) is true.

To complete the proof of Proposition B.2.1, one must make note of the fact that the maps $\hat{c}:[0,1] \times \hat{\mathfrak{B}} \rightarrow \mathfrak{B}$ of Eq. (B.2.2), and $m:[0,1] \times \hat{\mathfrak{B}}^{\delta} \rightarrow \mathfrak{B}^{\delta}$ of Lemma B.2.6 have the following properties: Neither increases $\mathfrak{U}^{\delta}$ as a function of $t \in[0,1]$ for fixed $b \in \hat{\mathfrak{B}}^{\delta}$; if $b=(A, \Phi) \in \hat{\mathfrak{B}}^{\delta}$ satisfies $\nabla_{A}^{*} \nabla_{A} \Phi=0$, then $\hat{c}(t, b)=b$ for all $t \in[0,1]$; if $b$ satisfies Eq. (B.2.1), then $m(t, b)=b$ for all $t \in[0,1]$; and if $b$ satisfies $\nabla_{A}^{*} \nabla_{A} \Phi=0$ then $m(1, b)=\left(A^{\prime}, \Phi^{\prime}\right)$ satisfies $\nabla_{A^{\prime}}^{*} \nabla_{A^{\prime}} \Phi^{\prime}=0$ too.

Construct $\tau_{4}:[0,1] \times \hat{\mathfrak{B}} \rightarrow \hat{\mathfrak{B}}$ as follows:

$$
\tau_{4}(t, b)= \begin{cases}\tau_{3}(3 t, b) & \text { if } t \in\left[0, \frac{1}{3}\right] \\ h_{1} \hat{c}\left(3 t-1, \tau_{3}(1, b)\right) & \text { if } t \in\left[\frac{1}{3}, \frac{2}{3}\right] \\ h_{2} m\left(3 t-2, h_{1} \hat{c}\left(1, \tau_{3}(1, b)\right)\right) & \text { if } t \in\left[\frac{2}{3}, 1\right]\end{cases}
$$


Here $h_{1}$ and $h_{2}$ are maps from $[0,1] \times \hat{\mathfrak{B}} \rightarrow \mathfrak{G}$ as provided by Lemma B.1.4. Let $F=\tau_{4}(1, G)$. Then apropos the comments of the preceding paragraph, $F$ satisfies Assertions (1) (4) of Lemma B.2.7. This $F$ also satisfies Assertions (3) and (4) of Proposition B.2.1. By choosing $\mu=\left[16\left(1+8 z\left(\mathfrak{H}_{\mathfrak{F}}+1\right)\right)\right]^{-1}(1+\varepsilon)^{-2} \varepsilon^{2}$, Assertions (2) and (3) of Lemma B.2.7 insure that this $F$ satisfies Assertions (1)-(4) of Proposition B.2.1.

\section{B.3. Convergence of Min-Max for $\mathfrak{A}^{\delta}$}

The proof of Proposition B.1.3 requires an assertion which gives a sufficient condition for a sequence in $\mathfrak{C}^{\delta}$ to contain a convergent subsequence. The proposition below provides this assertion by establishing a modified form of Condition $C$ for $\mathfrak{A}^{\delta}$ on $\mathfrak{C}^{\delta}$.

Proposition B.3.1. Let $\delta \in\left(0, \frac{1}{2}\right)$ and $n \in \mathbb{Z}$. Suppose that $\left\{c_{i}\right\} \subset \mathfrak{C}_{n}^{\delta}$ is a sequence with the following properties:

(1) $\mathfrak{H}^{\delta}\left(c_{i}\right) \geqq \mathfrak{H}^{\delta}\left(c_{i+1}\right) \rightarrow \mathfrak{U}_{\infty}^{\delta}$,

(2) As $i \rightarrow \infty,\left\|\nabla \mathfrak{A}_{i}^{\delta}\right\|_{*} \rightarrow 0$,

(3) For each $i$, write $c_{i}=(A, \Phi)$.

Then $\nabla_{A}^{*} \nabla_{A} \Phi=0$ and

(4) Equation (B.2.1) is satisfied by $(A, \Phi)$.

Then, there exists a sequence $\left\{g_{i}\right\} \subset \mathfrak{G}$ and a subsequence of $\left\{g_{i} c_{i}\right\}$ which converges strongly in the $L_{1 ; \text { loc }}^{2}$ topology on $\widetilde{\mathbb{C}}_{n}^{\delta}$ to a critical point, c, of $\mathfrak{A}^{\delta}$ on $\mathfrak{C}_{n}^{\delta}$ and $\mathfrak{I}^{\delta}(c)=\mathfrak{H}_{\infty}^{\delta}$.

The proof of this proposition occupies the remainder of this section. There are two aspects to the proof. The first aspect establishes the existence of the sequence $\left\{g_{i}\right\} \subset \mathfrak{G}$ for which a subsequence of $\left\{g_{i} c_{i}=\left(A_{i}, \Phi_{i}\right)\right\}$ has the property that $\left\{A_{i}\right\}$ converges strongly in $L_{1 ; \text { loc }}^{2}$ and $\left\{\Phi_{i}\right\}$ converges strongly in $L_{2 ; \text { loc }}^{2}$ to limits $A$ and $\Phi$. At the end, one concludes that $(A, \Phi) \in \mathfrak{C}^{\delta}$ is a critical point of $\mathfrak{A}^{\delta}$.

The second aspect to the proof of Proposition B.3.1 is to prove that for that locally convergent subsequence $\left\{\left(A_{i}, \Phi_{i}\right)\right\}$ of the preceding paragraph, the sequence of curvatures $\left\{\left((1+|x|)^{\delta} F_{A_{i}}, D_{A_{i}} \Phi_{i}\right)\right\}$ converges strongly in $L^{2}$.

The first aspect is summarized by

Lemma B.3.2. Let $\delta \in\left[0, \frac{1}{2}\right)$ and let $\left\{c_{i}\right\} \subset \mathbb{C}^{\delta}$ be a sequence which satisfies Assumptions (1)-(3) of Proposition B.3.1. There exists a sequence $\left\{g_{i}\right\} \subset(\mathfrak{5}$ and a subsequence of $\left\{g_{i} c_{i}\right\}$ with the following properties: For that subsequence, write $\left\{g_{i} c_{i}=\left(A_{i}, \Phi_{i}\right)\right\}$. Then $\left\{A_{i}\right\}$ converges strongly in $L_{1 ; \mathrm{loc}}^{2}\left(T^{*} \times \mathfrak{s u}(2)\right)$ to $A \in L_{1 ; \mathrm{loc}}^{2}\left(T^{*}\right.$ $\times \mathfrak{s u}(2))$; and $\left\{\Phi_{i}\right\}$ converges strongly in $L_{2 ; 1 \mathrm{loc}}^{2}(\operatorname{Ad} P)$ to $\Phi \in L_{2 ; \mathrm{loc}}^{2}(\operatorname{Ad} P)$. Further, $(A, \Phi) \in \mathfrak{C}^{\delta}$ and it is a critical point of $\mathfrak{U}^{\delta}$.

It should be stressed that Lemma B.3.2 holds for $\delta=0$.

Proof of Lemma B.3.2. In the $\delta=0$ case, Theorem 5.6 of [6, Part I] and an argument which is formally identical to the Appendix of [22] provide a specific 
choice of $\left\{g_{i}\right\} \subset\left(\mathfrak{b}\right.$ for which $\left\{g_{i} c_{i}\right\}$ has a subsequence $\left\{\left(A_{i}, \Phi_{i}\right)\right\}$ with the following properties: $\left\{A_{i}\right\}$ converges strongly in $L_{1 ; \text { loc }}^{2}$ to some $A$; likewise $\left\{\Phi_{i}\right\}$ converges strongly in $L_{1 ; \text { loc }}^{2}$ to some $\Phi$. Further, $(A, \Phi) \in \mathbb{C}$ and it is a critical point of $\mathfrak{A}$. These arguments generalize in a straightforward way to the $\delta>0$ cases. The convergence of $\left\{\Phi_{i}\right\}$ in $L_{2 ; \text { loc }}^{2}$ follows from Proposition B.3.3 of [9]; cf. the proof of Lemma A.4.4 of [9]. The details are left to the reader.

Proof of Proposition B.3.1. Now the Proposition is a direct corollary to Lemma B.3.2 and the lemma below.

Lemma B.3.3. Let $\delta \in\left(0, \frac{1}{2}\right)$. Let $\left\{c_{i}=\left(A_{i}, \Phi_{i}\right)\right\} \subset \mathbb{C}^{\delta}$ be a sequence which satisfies Assertions (1)-(4) of Proposition B.3.1. Assume in addition that $\left\{A_{i}\right\}$ converges strongly in $L_{1 ; \text { loc }}^{2}$ and that $\left\{\Phi_{i}\right\}$ converges strongly in $L_{2 ; \text { loc }}^{2}$. Then $\left\{c_{i}\right\}$ converges strongly in the $L_{1 ; \text { loc }}^{2}$ topology on $\mathbb{C}^{\delta}$.

According to Lemma B.3.2, the given sequence $\left\{\left(A_{i}, \Phi_{i}\right)\right\}$ converges weakly in $L_{1 ; \text { loc }}^{2} \times L_{2 ; \text { loc }}^{2}$ to $c=(A, \Phi)$ which is in $\mathfrak{C}^{\delta}$ and a critical point of $\mathfrak{A}^{\delta}$. To prove Lemma B.3.3 one need only show that $\lim \mathfrak{U}^{\delta}\left(c_{i}\right)=\mathfrak{A}^{\partial}(c)$ (see Sect. A.3). Note that Lemma A.4.5 of [9] then insures that the sequence of curvatures $\left\{\left((1+|x|)^{\delta} F_{A_{i}}, D_{A_{i}} \Phi_{i}\right)\right\}$ converges strongly in $L^{2}$.

Proof of Lemma B.3.3. It is convenient to establish the following notation: For each $i$, let $\left(F_{i}, G_{i}, \nabla_{i}, \nabla \mathfrak{A}_{i}^{\delta},\|\cdot\|_{i, \delta}\right)$ mean $\left(F_{A}, D_{A} \Phi, \nabla_{A}, \nabla \mathfrak{U}_{c}^{\delta},\|\cdot\|_{c, \delta}\right)$ for $c=(A, \Phi)=c_{i}$. Let $y_{i}=\left((1+|x|)^{\delta} F_{i}, G_{i}\right)$.

The first observation is that $\left\{y_{i}\right\}$ converges weakly in $L^{2}$, and strongly in $L_{\text {loc }}^{2}$. Assumption (3) of Proposition B.3.3 and Lemma A.4.1 of [9] insure that the sequences $\left\{(1+|x|)^{\delta} \nabla_{i} G_{i}\right\}$ in $L^{2}$ and $\left\{(1+|x|)^{\delta} G_{i}\right\}$ in $L^{6}$ converge weakly, but strongly in $L_{\text {loc }}^{2}$ and $L_{\text {loc }}^{6}$, respectively.

To obtain strong $L^{2}$ convergence from strong $L_{\text {loc }}^{2}$ convergence, Lemma A.4.6 of [9] will be the primary tool. Step 1 is to prove

Lemma B.3.4. Let $\left\{c_{i}=\left(A_{i}, \Phi_{i}\right)\right\} \subset \mathfrak{C}^{\delta}$ be as in Lemma B.3.3. Then the sequence $\left\{\left((1+|x|)^{\delta}\left[\Phi_{i}, F_{i}\right],\left[\Phi_{i}, G_{i}\right]\right)\right\}$ converges strongly in $L^{2}$.

Proof of Lemma B.3.4. Let $\beta \in C^{\infty}\left(\mathbb{R}^{3} ;[0,1]\right)$ be the bump function of Sect. A.3. For each $R \in(1, \infty)$, let $\beta_{R}(\cdot)=\beta((\cdot) / R)$.

For each $i$, let $\psi_{i}=\left(\left[\Phi_{i}, G_{i}\right], 0\right) \in \Gamma(Q)$. Be aware that Assumption (3) and the maximum principle imply that $\left|\Phi_{i}\right|<1$. Thus, $\psi_{i} \in L^{2}(Q)$. Further, $\nabla_{i} \psi_{i}=\left(\left[\Phi_{i}, \nabla_{i} G_{i}\right], 0\right)+\left(\left[G_{i}, G_{i}\right], 0\right)$, and so $(1+|x|)^{\delta} \nabla_{i} \psi_{i}$ is also uniformly bounded in $L^{2}$. Indeed,

$$
\left|(1+|x|)^{\delta}\left[G_{i}, G_{i}\right]\right| \leqq 2(1+|x|)^{-\delta / 2}\left|G_{i}\right|^{1 / 2} \cdot\left((1+|x|)^{\delta}\left|G_{i}\right|\right)^{3 / 2},
$$

and so Hölder's inequality bounds $\left\|(1+|x|)^{\delta}\left[G_{i}, G_{i}\right]\right\|_{2}^{2}$ by $4\left\|G_{i}\right\|_{2} \cdot\left\|(1+|x|)^{\delta} G_{i}\right\|_{6}^{3}$.

For $R \in[1, \infty)$, the preceding discussion implies that although $\left(1-\beta_{R}\right) \psi_{i}$ is not compactly supported, it is the limit of such with respect to the norm $\|\cdot\|_{i, \delta}$ and additionally, $\left\{\left\|\left(1-\beta_{R}\right) \psi_{i}\right\|_{i, \delta}\right.$ is bounded uniformly in $R$ and $i$. Therefore, Assumption (2), and Eq. (A.4.5) imply that

$$
\lim _{i \rightarrow \infty}\left|\nabla \mathfrak{U}_{i}^{\delta}\left(\left(1-\beta_{R}\right) \psi_{i}\right)\right|=0 .
$$


In long hand, $\nabla \mathfrak{H}_{i}^{\delta}\left(\left(1-\beta_{R}\right) \psi_{i}\right)$ is

$$
\begin{aligned}
& \left\langle\left(1+|x|^{2}\right)^{\delta}\left(1-\beta_{R}\right)\left[G_{i}, G_{i}\right], F_{i}\right\rangle_{2}-\left\langle\left(1+|x|^{2}\right)^{\delta} d \beta_{R} \wedge\left[\Phi_{i}, G_{i}\right], F_{i}\right\rangle_{2} \\
& \quad+\left\langle\left(1+|x|^{2}\right)^{\delta}\left(1-\beta_{R}\right)\left[\Phi_{i},\left[F_{i}, \Phi_{i}\right]\right], F_{i}\right\rangle_{2}+\left\langle\left(1-\beta_{R}\right)\left[\left[\Phi_{i}, G_{i}\right], \Phi_{i}\right], G_{i}\right\rangle_{2} .
\end{aligned}
$$

In deriving Eq. (B.3.3), the identity $D_{A} D_{A} \Phi=\left[F_{A}, \Phi\right]$ has been used. Equations (B.3.1-3) after rearranging imply the following: Given $\varepsilon>0$, there exists $i(\varepsilon)<\infty$ which is independent of $R$ such that for all $i>i(\varepsilon)$,

$$
\begin{aligned}
& \left\langle\left(1-\beta_{R}\right)\left(1+|x|^{2}\right)^{\delta}\left[\Phi_{i}, F_{i}\right],\left[\Phi_{i}, F_{i}\right]\right\rangle_{2}+\left\langle\left(1-\beta_{R}\right)\left[\Phi_{i}, G_{i}\right],\left[\Phi_{i}, G_{i}\right]\right\rangle_{2} \\
& \quad<\varepsilon+z \cdot\left(R^{-\delta / 2}\left\|G_{i}\right\|_{2}\left\|(1+|x|)^{\delta} G_{i}\right\|_{6}\left\|(1+|x|)^{\delta} F_{i}\right\|_{2}+R^{-1+2 \delta}\left\|G_{i}\right\|_{2}\left\|F_{i}\right\|_{2}\right) .
\end{aligned}
$$

Because $\delta \in\left(0, \frac{1}{2}\right)$, Eq. (B.3.4) and the uniform bounds on $\left\{\left\|(1+|x|)^{\delta} F_{i}\right\|_{2},\left\|G_{i}\right\|_{2}\right.$, $\left.\left\|(1+|x|)^{\delta} G_{i}\right\|_{6}\right\}$ imply via Lemma A.4.6 of [9] the convergence assertion of Lemma B.3.4.

Step 2 in the proof of Lemma B.3.3 is to prove

Lemma B.3.5. Let $\left\{c_{i}=\left(A_{i}, \Phi_{i}\right)\right\} \subset \mathbb{C}^{\delta}$ be as in Lemma B.3.3. Then the sequence $\left\{\left(1-\left|\Phi_{i}\right|\right)\right\}$ converges strongly in $\bar{C}^{0}\left(\mathbb{R}^{3}\right)$. In particular, given $\varepsilon>0$, there exists $R<\infty$ and $i(\varepsilon)<\infty$ such that for all $i>i(\varepsilon), \sup _{|x|>R}|1-| \Phi_{i}|(x)|<\varepsilon$.

Proof of Lemma B.3.5. Since $\left\{(1+|x|)^{\delta} G_{i}\right\}$ is uniformly bounded in $L^{6},\left\{G_{i}\right\}$ is strongly convergent in $L^{6}$ (Lemma A.4.6 of [9]) and so $\left\{d\left(1-\left|\Phi_{i}\right|\right)\right\}$ is strongly convergent in $L^{6}\left(T^{*}\right)$ (Kato's inequality, Sect. A.4 of [9]). The lemma follows by demonstrating the strong convergence of $\left\{\left(1-\mid \Phi_{i}\right)\right\}$ in $L^{p}$ for some $1 \leqq p<\infty$ (cf. Lemma A.4.4 of [9]). Now, because $\left\{\left\|G_{i}\right\|_{2}\right\}$ is uniformly bounded [Assumption (1)], so are $\left\{\left\|d\left(1-\left|\Phi_{i}\right|\right)\right\|_{2}\right\}$ and $\left\{\left\|\left(1-\left|\Phi_{i}\right|\right)\right\|_{6}\right\}$ (Lemma A.4.1 of [9]). Now, $d\left(1-\left|\Phi_{i}\right|\right)^{3}=3\left(1-\left|\Phi_{i}\right|\right)^{2} d\left(1-\left|\Phi_{i}\right|\right)$, and so Lemma A.4.1 of [9] implies that for any $R \in[1, \infty)$,

$$
\left\|\left(1-\beta_{R}\right)\left(1-\left|\Phi_{i}\right|\right)^{3}\right\|_{6} \leqq z\left[\left\|\left(1-\left|\Phi_{i}\right|\right)^{2}\left(1-\beta_{R}\right) d\left(1-\left|\Phi_{i}\right|\right)\right\|_{2}+\left\|\left(1-\left|\Phi_{i}\right|\right)^{6} d \beta_{R}\right\|_{2}\right] .
$$

The preceding equation implies the inequality

$$
\left\|\left(1-\beta_{R}\right)\left(1-\left|\Phi_{i}\right|\right)^{3}\right\|_{6} \leqq z\left\{\left\|\left(1-\beta_{R}\right) d\left(1-\left|\Phi_{i}\right|\right)\right\|_{6}+R^{-1}\right\} .
$$

Finally, the strong convergence of $d\left(1-\left|\Phi_{i}\right|\right)$ in $L^{6}$ and Eq. (B.3.5) imply with Lemma A.4.6 of $[9]$ that $\left\{\left(1-\left|\Phi_{i}\right|\right)\right\}$ converges strongly in $L^{18}\left(\mathbb{R}^{3}\right)$. As previously remarked, $\left\{\left(1-\left|\Phi_{i}\right|\right)\right\}$ converges strongly in $\bar{C}^{0}\left(\mathbb{R}^{3}\right)$. The second claim of Lemma B.3.5 is a direct consequence of this last conclusion.

Step 3 of the proof of Proposition B.3.1 proves

Lemma B.3.6. Let $\left\{c_{i}=\left(A_{i}, \Phi_{i}\right)\right\} \subset \mathfrak{C}^{\delta}$ be as in Lemma B.3.3. Then the sequence $\left\{\left(\Phi_{i}, \nabla_{i} \Phi_{i}\right)\right\}$ converges strongly in $L^{2}\left(T^{*}\right)$.

Proof of Lemma B.3.6. For each $i$, let $w_{i}=\frac{1}{2}\left(1-\left|\Phi_{i}\right|^{2}\right)$. Due to Assumption (3) of Proposition B.3.1,

$$
-\Delta w_{i}=\left|G_{i}\right|^{2}
$$


Also, $\left\{d w_{i}\right\}$ is uniformly bounded in $L^{2}\left(T^{*}\right)$ and $\left\{w_{i}\right\}$ is uniformly bounded in $L^{6}\left(T^{*}\right)$. It is therefore permissable to multiply both sides of Eq. (B.3.6) by $w_{i}$ and then integrate both sides over $\mathbb{R}^{3}$. The resulting expression from the left-hand side can be manipulated by an integration by parts, and no boundary terms arise. One obtains

$$
\left\langle d w_{i}, d w_{i}\right\rangle_{2}=\left\langle w_{i} G_{i}, G_{i}\right\rangle_{2} .
$$

Let $(A, \Phi) \in \mathfrak{C}^{\delta}$ be the limit of $\left\{\left(A_{i}, \Phi_{i}\right)\right\}$ in the sense given by Lemma B.3.2. Since $\nabla_{A}^{2} \Phi=0$, if one sets $w=\frac{1}{2}\left(1-|\Phi|^{2}\right)$ and $G=\nabla_{A} \Phi$, then also

$$
\langle d w, d w\rangle=\langle w G, G\rangle_{2} .
$$

Because $\left\{G_{i}\right\}$ converges strongly in $L_{\mathrm{loc}}^{2}$ to $G$, and because of Lemma B.3.5,

$$
\lim _{i \rightarrow \infty}\left\langle w_{i} G_{i}, G_{i}\right\rangle_{2}=\langle w G, G\rangle_{2} .
$$

Because $d w_{i}=-\left(\Phi_{i}, G_{i}\right)$, Eqs. (B.3.7-9) imply that

$$
\lim _{i \rightarrow \infty}\left\|\left(\Phi_{i}, G_{i}\right)\right\|_{2}=\|(\Phi, G)\|_{2} .
$$

The strong convergence of $\left\{\left(\Phi_{i}, G_{i}\right)\right\}$ to $(\Phi, G)$ in $L^{2}$ is now observed as a direct consequence of Lemma A.4.5 of [9] and Eq. (B.3.10).

For the final step to the proof of Lemma B.3.3, one should observe that Lemmas B.3.4-B.3.6 imply that $\left\{G_{i}\right\}$ converges strongly in $L^{2}$ to $\{G\}$. Since $\left\{F_{i}\right\}$ converges strongly in $L^{2}$ to $\left\{F_{A}\right\}$, the convergence of $\left\{c_{i}\right\}$ in the $L_{1 ; \text { loc }}^{2}$ topology on $\mathbb{C}$ has now been established. To prove that convergence is in the $L_{1 ; \text { loc }}^{2}$ topology on $\mathfrak{C}^{\delta}$, one must utilize Assumption (4) of Proposition B.3.1. For this, one requires

Lemma B.3.7. For $\delta \in\left[0, \frac{1}{2}\right)$, let $(A, \Phi) \in \mathfrak{C}^{\delta}$ be a critical point of $\mathfrak{A}^{\delta}$. Then $(A, \Phi)$ satisfies Eq. (B.2.1).

Proof of Lemma B.3.7. The case $\delta=0$ is proved as Corollary II.2.2 of [11]. The case $\delta \in\left(0, \frac{1}{2}\right)$ is established as Corollary D.1.5. Certain a priori estimates on critical points of $\mathfrak{U}^{\delta}$ in $\mathfrak{C}^{\delta}$ that one requires can be found in Sects. C.2-4, here. (Warning: The definition of a critical point given in Sect. A.4 here precludes concluding this lemma directly from Lemma B.2.5.)

Proof of Lemma B.3.3 (completion). Lemma B.3.7 and the strong $L^{2}$ convergence of $\left\{\left(F_{i}, G_{i}\right)\right\}$ to $\left(F_{A}, \nabla_{A} \Phi\right)$ and Assumption (4) of Proposition B.3.1 imply

$$
\lim _{i \rightarrow \infty}\left\|(1+|x|)^{\delta} F_{i}\right\|_{2}=\left\|(1+|x|)^{\delta} F_{A}\right\|_{2} .
$$

Thus, $\lim _{i \rightarrow \infty} \mathfrak{H}^{\delta}\left(c_{i}\right)=\mathfrak{H}^{\delta}(c)$ and $\left\{c_{i}\right\}$ converges strongly in the $L_{1 ; \text { loc }}^{2}$ topology on $\mathfrak{C}^{\delta}$.

\section{B.4. Final Arguments for $\mathfrak{Q}^{\boldsymbol{\delta}}$}

The purpose of this section is to complete the proof of Proposition B.1.3 by sewing together the assertions of Propositions B.2.1 and B.3.1. 
Proof of Proposition B.1.3. Let $\mathfrak{F}$ be as assumed in Proposition B.1.3 and let $\delta \in\left(0, \frac{1}{2}\right)$. For each $m \in\{1,2, \ldots\}$, let $\left(F_{m}, c_{m}\right) \in Y^{\delta}(\mathfrak{F})$ be the data provided by Proposition B.2.1 with $\varepsilon=m^{-1}$ there.

Consider the sequence $\left\{c_{m}\right\} \subset \hat{B}^{\delta}$. According to Proposition B.2.1, this sequence satisfies Assumptions (1)-(4) of Proposition B.3.1 with $\mathfrak{H}_{\infty}^{\delta}=\mathfrak{H}_{\mathfrak{F}}^{\delta}$. Proposition B.3.1 asserts that this sequence has a subsequence, $\left\{c_{i}\right\}$, for which a sequence $\left\{g_{i}\right\} \subset(\mathfrak{b}$ exists such that $\left\{g_{i} c_{i}\right\}$ converges strongly in the $L_{1 ; \text { loc }}^{2}$ topology on $\mathbb{C}^{\delta}$ to a critical point of $\mathfrak{U}^{\delta}$. A priori, this critical point, $c$, satisfies $\mathfrak{U}^{\delta}(c)=\mathfrak{U}_{\mathfrak{Z}}^{\delta}$.

Now consider

Lemma B.4.1. Let $\left\{\left(F_{i}, c_{i}\right)\right\} \subset Y^{\delta}(\mathfrak{F}),\left\{g_{i}\right\} \subset \mathfrak{G}$ and $c \in \mathbb{C}^{\delta}$ be such that $\left\{g_{i} c_{i}\right\}$ converges to $c$ in the $L_{1 ; \text { loc }}^{2}$ topology on $\mathbb{C}^{\delta}$. Then there exists $\left\{\left(F_{i}^{\prime}, b_{i}\right)\right\} \subset Y^{\delta}(\mathfrak{F})$ and $\left\{h_{i}\right\} \subset(\mathfrak{F}$ such that $\left\{h_{i} b_{i}\right\}$ converges to $c$ in the $C^{\infty}$ topology on $\mathbb{C}^{\delta}$.

Given Lemma B.4.1, the proof of Proposition B.1.3 is completed by observing that because $\pi: \mathfrak{C}^{\delta} \rightarrow \mathfrak{B}^{\delta}$ is continuous, $\left\{\pi\left(h_{i} b_{i}\right)\right\}$ converges to $\pi(c)$ in $\mathfrak{B}^{\delta}$. But, for each $i$, there exists $l_{i} \in \mathrm{SU}(2)$ such that $l_{i} \cdot \pi\left(h_{i} b_{i}\right)=b_{i}$. Since $\mathrm{SU}(2)$ is compact, $\left\{l_{i}\right\}$ has a convergent subsequence with limit $l$. Then $l \cdot \pi(c) \equiv c^{\delta}(\mathfrak{F}) \in \mathfrak{B}^{\delta}$ and the corresponding subsequence of $\left\{b_{i}\right\}$ converges to $c^{\delta}(\mathfrak{F})$ in $\hat{\mathfrak{B}}^{\delta}$.

Proof of Lemma B.4.1. Let $\beta:[0, \infty) \rightarrow[0,1]$ be the bump function of Sect. A.3 and for each $n \in\{1,2, \ldots\}$, let $\beta_{n}(\cdot)=\beta((\cdot) / n)$.

The $L_{1 ; \text { loc }}^{2}$ convergence of $\left\{g_{i} c_{i}\right\}$ to $c$ means that one can choose for each $i$, an integer $n(i)$ such that $\left\{g_{i}\left(c_{i}+g_{i}^{-1} \beta_{n(i)}\left(c-g_{i} c_{i}\right)\right)\right\}$ converges to $c$ in the $C^{\infty}$ topology on $\mathfrak{C}^{\delta}$ and such that for each $i$ and $t \in[0,1]$ (see Proposition A.4.3),

$$
\left|\mathfrak{H}^{\delta}\left(c_{i}+g_{i}^{-1} t \beta_{n(i)}\left(c-g_{i} c_{i}\right)\right)-\mathfrak{A}^{\delta}\left(c_{i}\right)\right|<\frac{1}{2} i^{-1} .
$$

For each $i$, there exists a neighborhood $\mathfrak{B}_{i} \subset \mathfrak{B}$ of $c_{i}$ and a function $f_{i}: \mathfrak{B} \rightarrow[0,1]$ such that (1) $f_{i} \equiv 0$ on $\mathfrak{\mathfrak { B }} \backslash \mathfrak{B}_{i},(2) f_{i}\left(c_{i}\right)=1$ and (3) for all $(t, b) \in[0,1] \times \mathfrak{B}_{i}$,

$$
\left|\mathfrak{H}^{\delta}\left(b+g_{i}^{-1} t f_{i}(b) \beta_{n(i)}\left(c-g_{i} c_{i}\right)\right)-\mathfrak{Q}^{\delta}(b)\right|<\frac{1}{2} i^{-1} .
$$

The convexity of $\mathfrak{A}^{\delta}$ with respect to compactly supported changes in $\Phi$ allows one to add to each $c_{i}$ a compactly supported $\psi_{i}=\left(0, \phi_{i}\right)$ with the following properties: First, $\left\{\phi_{i}\right\}$ converges to zero in $L_{1}^{2}(\operatorname{Ad} P) \cap C^{\infty}(\operatorname{Ad} P)$ and second,

$$
\mathfrak{U}^{\delta}\left(c_{i}\right)+\frac{1}{2} i^{-1}<\mathfrak{A}^{\delta}\left(c_{i}+\psi_{i}+g_{i}^{-1} \beta_{n(i)}\left(c-g_{i} c_{i}\right)\right)<\mathfrak{A}^{\delta}\left(c_{i}\right)+i^{-1} .
$$

With a suitably chosen $f_{i}^{\prime}: \hat{\mathfrak{B}} \rightarrow[0,1]$ with $f_{i}^{\prime}\left(c_{i}\right)=1$ and with support on $\mathfrak{B}_{i}$, one can require that for all $b \in F_{i}$,

$$
\mathfrak{A}^{\delta}\left(b+f_{i}^{\prime}(b) \psi_{i}+g_{i}^{-1} f_{i}(b) \beta_{n(i)}\left(c-g_{i} c_{i}\right)\right)<\mathfrak{A}^{\delta}\left(c_{i}+\psi_{i}+g_{i}^{-1} \beta_{n(i)}\left(c-g_{i} c_{i}\right)\right) .
$$

Now, for each $i$, define a map $L_{i}:[0,1] \times \mathfrak{\mathfrak { B }} \rightarrow \mathfrak{C}$ by sending $(t, b)$ to

$$
L_{i}(t, b)=b+t f_{i}^{\prime}(b) \psi_{i}+g_{i}^{-1} t f_{i}(b)\left(c-g_{i} c_{i}\right) .
$$

Note that $L_{i}(0, \cdot)=\left.\operatorname{Identity}\right|_{\hat{\mathfrak{B}}}$, and that $L_{i}$ maps $[0,1] \times \hat{\mathfrak{B}}^{\delta}$ into $\mathbb{C}^{\delta}$.

Define $F_{i}^{\prime}$ to be $\left(h \cdot L_{i}\right)\left(1, F_{i}\right)$ with $h$ given by Lemma B.1.4. Due to Eq. (B.4.1), $\left(F_{i}^{\prime}, h \cdot\left(c_{i}+\psi_{i}+g_{i}^{-1} \beta_{n(i)}\left(c-g_{i} c_{i}\right)\right)\right) \in Y^{\delta}(\mathfrak{F})$ and by construction satisfies Lemma B.4.1's requirements. 


\section{C.1. A Priori Estimates}

For the proof of Theorem A.1.2, it is necessary to establish the following proposition.

Proposition C.1.1. Let $n \in \mathbb{Z}$, and let $\mathfrak{F}$ be a homotopy invariant family of compact subsets of $\hat{\mathfrak{B}}_{n}$. Let $\left\{c^{\delta}(\mathfrak{F}) \in \hat{\mathfrak{B}}_{n}^{\delta} ; \delta \in\left(0, \frac{1}{2}\right)\right\}$ be the set of configurations that is provided by Proposition B.1.3. If $\mathfrak{U}_{\mathfrak{F}}>4 \pi|n|$, then there exists a decreasing sequence $\Lambda=\left\{\delta_{1}, \delta_{2}, \ldots\right\} \subset\left(0, \frac{1}{2}\right)$ with limit zero such that the sequence $\left\{c^{\delta}(\mathfrak{F}): \delta \in \Lambda\right\}$ converges in $\mathfrak{B}_{n}$ to $c(\mathfrak{F})$, a critical point of $\mathfrak{A}$ with critical value $\mathfrak{U}_{\mathfrak{F}}$.

The proof of this proposition constitutes Sects. C.1-D.4, and the full argument is summarized in Sect. D.4. A crucial part of the proof is to obtain good a priori estimates on the configurations $c^{\delta}(\mathfrak{F})$. These are derived from the variational equations of $\mathfrak{U}^{\delta}$ : If $(A, \Phi) \in \mathfrak{C}^{\delta}$ is a critical point of $\mathfrak{U}^{\delta}$, then

$$
\begin{gathered}
* D_{A} *\left(1+|x|^{2}\right)^{\delta} F_{A}+\left[\Phi, D_{A} \Phi\right]=0, \\
D_{A} * D_{A} \Phi=0 .
\end{gathered}
$$

If one considers Eq. (C.1.1) as equations for the curvatures, $\left(F_{A}, D_{A} \Phi\right)$, then it is useful to consider also the identities

$$
\begin{gathered}
D_{A} F_{A}=0, \\
D_{A} D_{A} \Phi+\left[\Phi, F_{A}\right]=0 .
\end{gathered}
$$

Together, Eqs. (C.1.1-2) form an elliptic system for $\left(F_{A}, D_{A} \Phi\right)$. This fact is made transparent by using these equations to derive the following second order equations for $\left(F_{A}, D_{A} \Phi\right)$ : For notational convenience, let $\sigma=\left(1+|x|^{2}\right)^{1 / 2}$ and let $(f, g)=\left(* \sigma^{\delta} F_{A}, D_{A} \Phi\right) \in \underset{2}{\times} \Gamma\left(\operatorname{Ad} P \otimes T^{*}\right)$. Also, $\mathbf{x}=\frac{1}{2} d|x|^{2}$.

$$
\begin{aligned}
-\nabla_{A}^{*} \nabla_{A} f & +\sigma^{-2 \delta}[\Phi,[\Phi, f]]-\sigma^{-\delta} *([f, f]+[g, g]) \\
& +\delta * D_{A} *\left(\sigma^{-2} \mathbf{x} \wedge f\right)-\delta D_{A} *\left(\sigma^{-2} \mathbf{x} \wedge * f\right)+\delta \sigma^{-2-\delta} *(\mathbf{x} \wedge[\Phi, g])=0 \\
-\nabla_{A}^{*} \nabla_{A} g & +\sigma^{-2 \delta}[\Phi,[\Phi, g]]-\sigma^{-\delta} *([f, g]+[g, f]) \\
& +\delta \sigma^{-2-\delta} *(\mathbf{x} \wedge[\Phi, f])=0 .
\end{aligned}
$$

These equations are derived as in the $\delta=0$ case which is presented in Ch. IV.9 of [11].

The estimates that are required for Proposition C.1.1 are formally similar to those derived for solutions to Eq. (A.1.1) in [9, Chap. IV]. The difference here is that more specific information is required. For this purpose, introduce for each $\kappa>0$, and $c=(A, \Phi) \in \mathfrak{C}$, the set $U[c](\kappa)$. (See Definition C.2.8 of [9].) This set is defined by first setting

$$
\hat{U}[c](\kappa)=\left\{x \in \mathbb{R}^{3}: \int_{|x-y|<1} d^{3} y\left(\left|F_{A}\right|^{2}+\left|D_{A} \Phi\right|^{2}\right)>\kappa\right\},
$$

and then by setting

$$
U[c](\kappa)=\left\{x \in \mathbb{R}^{3}: \operatorname{dist}(x, \hat{U}[c](\kappa))<1\right\} .
$$


Three important and obvious properties of $U[c](\kappa)$ are summarized by the next lemma.

Lemma C.1.2. For each $c \in \mathbb{C}$ and $\kappa>0$ define the set $U[c](\kappa)$ by Eq. (C.1.4). Then

(1) $U[c](\kappa)$ is bounded.

(2) The number of path components of $U[c](\kappa), n[c](\kappa)$, is bounded by $\kappa^{-1} \mathfrak{U}(c)$.

(3) The diameter of any path component of $U[c](\kappa)$ is bounded by $4 \kappa^{-1} n[c](\kappa)^{-1} \mathfrak{U}(c)$.

Proof of Lemma C.1.2. See the proof of Lemma C.2.9 of [9].

The first set of estimates that are required for the proof of Proposition C.1.2 are the sup norm estimates below.

Lemma C.1.3. Let $\delta \in\left[0, \frac{1}{2}\right)$ and let $c=(A, \Phi) \in \mathbb{C}^{\delta}$ be a solution to Eq. (C.1.1). Then both $\|f\|_{\infty}$ and $\|g\|_{\infty}$ are bounded uniformly in $\delta$ knowing a priori only a bound for $\mathfrak{U}^{\delta}(c)$.

The second set of estimates states that $f$ and $g$ are pointwise small outside of $U[c](\kappa)$.

Lemma C.1.4. There exist continuous functions $\lambda:[0, \infty) \times(0,1] \rightarrow(0,1]$ and $R:[0, \infty) \times(0,1] \rightarrow[0, \infty)$ with the following significance: Let $\delta \in\left[0, \frac{1}{4}\right)$ and let $c=(A, \Phi) \in \mathbb{C}^{\delta}$ be a solution to $E q$. (C.1.1). Given $\varepsilon \in(0,1]$, let $U=U[c]\left(\lambda\left(\mathfrak{A}^{\delta}(c), \varepsilon\right)\right)$. At each $x \in \mathbb{R}^{3}$ with $\operatorname{dist}(x, U)>R\left(\lambda\left(\mathfrak{U}^{\delta}(c), \varepsilon\right)\right)$,

(1) $\sigma^{2 \delta}(|f|+|g|)(x)<\varepsilon$,

(2) $\sigma^{2 \delta}\left(\left|\nabla_{A} f\right|+\left|\nabla_{A} g\right|\right)(x)<\varepsilon$,

(3) $1-|\Phi|(x)<\varepsilon$.

The remainder of this section contains the proofs of Lemmas C.1.3 and C.1.4.

Proof of Lemma C.1.3. The $\delta=0$ case is stated as Proposition IV.10.6 of [11]. The case $\delta>0$ is proved by mimicking the $\delta=0$ proof. The details are nearly identical and omitted. One obtains the uniform estimate

$$
\|f\|_{\infty}^{2}+\|g\|_{\infty}^{2}<\zeta\left(\mathfrak{U}^{\delta}(c)\right)
$$

with $\zeta(\cdot)$ continuous and independent of $c \in \mathfrak{C}^{\delta}$ and $\delta \in\left[0, \frac{1}{2}\right)$.

To prove Lemma C.1.4, introduce the notation $\Psi=(f, g) \in \Gamma\left(\underset{2}{\oplus}\left(\operatorname{Ad} P \times T^{*}\right)\right)$. Proof of Assertion (1) of Lemma C.1.4. Observe that contracting both sides of Eq. (C.1.3) by $\Psi$ yields the scalar equation $d^{*} d \frac{|\Psi|^{2}}{2}+\left(\left|\nabla_{A} \Psi\right|^{2}+\sigma^{-2 \delta}|[\Phi, \Psi]|^{2}\right) \leqq z\left\{\sigma^{-\delta}|\Psi|^{3}+\delta \sigma^{-1-\delta}|\Psi|^{2}+\delta \sigma^{-1}|\Psi|\left|\nabla_{A} \Psi\right|\right\}$, 
where $z<\infty$ is independent of $c \in \mathfrak{C}^{\delta}$ and $\delta \in\left[0, \frac{1}{2}\right)$. By replacing $z$ by another constant independent of $c \in \mathfrak{C}^{\delta}$ and $\delta \in\left[0, \frac{1}{2}\right)$, one obtains

$$
d^{*} d\left(\sigma^{4 \delta} \frac{|\Psi|^{2}}{2}\right)+\frac{1}{2}\left(\sigma^{4 \delta}\left|\nabla_{A} \Psi\right|^{2}+\sigma^{2 \delta}|[\Phi, \Psi]|\right)^{2} \leqq z\left\{\sigma^{3 \delta}|\Psi|^{3}+\delta \sigma^{-1+3 \delta}|\Psi|^{2}\right\} .
$$

Introduce for each $R \in[1, \infty)$ the function $\beta_{R}(\cdot)=\beta((\cdot) / R)$ with $\beta$ the bump function from Sect. A.3. Introduce the Green's function $(4 \pi|(\cdot)-y|)^{-1}$ for $d^{*} d$ with singularity at $y \in \mathbb{R}^{3}$.

Multiply both sides of Eq. (C.1.6) by $\beta_{R}^{2}(4 \pi|(\cdot)-y|)^{-1}$ and integrate over $\mathbb{R}^{3}$. Then integrate by parts to obtain

$$
\begin{aligned}
& \beta_{R}^{2} \sigma^{4 \delta} \frac{|\Psi|^{2}}{2}(y)+\frac{1}{8 \pi} \int \frac{\beta_{R}^{2}}{|(\cdot)-y|}\left(\sigma^{4 \delta}\left|\nabla_{A} \Psi\right|^{2}+\sigma^{2 \delta}|[\Phi, \Psi]|^{2}\right) \\
& \quad \leqq \frac{z}{4 \pi}\left[\int \frac{1}{|(\cdot)-y|} \beta_{R}^{2}\left(\sigma^{3 \delta}\left|\Psi^{3}\right|+\delta \sigma^{-1+3 \delta}|\Psi|^{2}\right)+\int\left(\frac{\left|d^{*} d \beta_{R}^{2}\right|}{|(\cdot)-y|}+\frac{\left|d \beta_{R}\right|}{|(\cdot)-y|^{2}}\right) \sigma^{4 \delta}|\Psi|^{2}\right] .
\end{aligned}
$$

The right-hand side of Eq. (C.1.7) is estimated in the following way: First, because $\left|d^{*} d \beta_{R}^{2}\right| \sigma^{4 \delta} \leqq R^{-2+4 \delta}$ and $\left|d \beta_{R}\right| \sigma^{4 \delta}<R^{-1+4 \delta}$, the last term on the right-hand side of Eq. (C.1.7) is bounded by

$$
z \cdot R^{-1+4 \delta} \int\left(\frac{1}{|(\cdot)-y|}+\frac{1}{|(\cdot)-y|^{2}}\right)|\Psi|^{2} \leqq z \cdot R^{-1+4 \delta}\left(\mathfrak{H}^{\delta}(c)+\zeta\left(\mathfrak{A}^{\delta}(c)\right)\right),
$$

where the factor $\zeta\left(\mathfrak{H}^{\delta}(c)\right)$ is due to Eq. (C.1.4). The first term on the right-hand side of Eq. (C.1.7) is bounded by breaking the integral into the part $|(\cdot)-y|>1$ and the part $|(\cdot)-y|<1$. The result is the uniform bound

$$
\zeta\left(\mathfrak{A}^{\delta}(c)\right)\left(1+\left\|\beta_{R} \sigma^{2 \delta} \Psi\right\|_{\infty}\right)^{7 / 4} .
$$

With Eqs. (C.1.8-9), the right-hand side of Eq. (C.1.7) is bounded independently of $y \in \mathbb{R}^{3}$. Take the sup over $y \in \mathbb{R}^{3}$ on the left-hand side of Eq. (C.1.7). On the left, $\left\|\beta_{R} \sigma^{2 \delta} \Psi\right\|_{\infty}^{2}$ appears while on the right, $\left\|\beta_{R} \sigma^{2 \delta} \Psi\right\|_{\infty}^{7 / 4}$ appears. Therefore, one obtains the uniform estimate, below, with $\zeta(\cdot)$ continuous, and independent of $R$, $c \in \mathfrak{C}^{\delta}$ and $\delta \in\left[0, \frac{1}{4}\right)$ :

$$
\left\|\beta_{R} \sigma^{2 \delta} \Psi\right\|_{\infty}^{2}+\frac{1}{16 \pi} \sup _{y} \int \beta_{R}^{2} \frac{1}{|(\cdot)-y|}\left(\sigma^{4 \delta}\left|\nabla_{A} \Psi\right|^{2}+\sigma^{2 \delta}|[\Phi, \Psi]|^{2}\right) \leqq \zeta\left(\mathfrak{H}^{\delta}(c)\right) .
$$

As the right-hand side of Eq. (C.1.10) is independent of $R$, one obtains, first,

$$
\left\|\sigma^{2 \delta} \Psi\right\|_{\infty}^{2}<\zeta\left(\mathfrak{H}^{\delta}(c)\right)
$$

and second, for each $y \in \mathbb{R}^{3}$,

$$
\sigma^{4 \delta}|\Psi|^{2}(y)+\frac{1}{4 \pi} \int \frac{1}{|(\cdot)-y|} \sigma^{4 \delta}\left|\nabla_{A} \Psi\right|^{2} \leqq z \cdot \int \frac{1}{|(\cdot)-y|}\left(\sigma^{3 \delta}|\Psi|^{3}+\delta \sigma^{-1+3 \delta}|\Psi|^{2}\right) .
$$


To obtain Assertion (1) of Lemma C.1.4, fix $\kappa>0$ and consider for $R \in(0, \infty)$ a point $y \in \mathbb{R}^{3}$ with

$$
\operatorname{dist}(y, U[c](\kappa))>R .
$$

For such $y$, the right-hand side of Eq. (C.1.12) is bounded by

$$
\begin{aligned}
& z R^{-1 / 4}\left(\left\|\sigma^{2 \delta} \Psi\right\|_{\infty}^{3 / 2}+1\right) \mathfrak{P}^{\delta}(c)^{3 / 4} \\
& \quad+z \cdot\left(\left\|\sigma^{2 \delta} \Psi\right\|_{\infty}^{2}+1\right) \int_{|x-y|<R} d^{3} x \frac{1}{|x-y|}\left(\left|F_{A}\right|(x)+\left|D_{A} \Phi\right|(x)\right),
\end{aligned}
$$

and therefore

$$
\sigma^{4 \delta}|\Psi|^{2}(y)+\frac{1}{4 \pi} \int \frac{1}{|(\cdot)-y|} \sigma^{4 \delta}\left|\nabla_{A} \Psi\right|^{2} \leqq \zeta\left(\mathfrak{Q}^{\delta}(c)\right)\left(R^{-1 / 4}+\kappa R^{2}\right) .
$$

Here, $\zeta(\cdot)$ is continuous and independent of $y$ satisfying Eq. (C.1.13), $R, c \in \mathbb{C}^{\delta}$, and $\delta \in\left[0, \frac{1}{4}\right)$. For Assertion (1) of Lemma C.1.4, take $\lambda\left(\mathfrak{H}^{\delta}(c), \varepsilon\right)$ $=\left[\frac{1}{2} \varepsilon^{2} \zeta\left(\mathfrak{H}^{\delta}(c)\right)^{-1}\right]^{9}$ and take $R\left(\mathfrak{H}^{\delta}(c), \varepsilon\right)=\lambda^{-4 / 9}$.

Proof of Assertion (2) of Lemma C.1.4. Take the covariant derivative of Eq. (C.1.3). After commuting covariant derivatives, one obtains

$$
-\nabla_{A}^{*} \nabla_{A}\left(\nabla_{A} \Psi\right)+\Lambda(\Psi) \nabla_{A} \Psi+\nabla_{A} Q=0 .
$$

Here, $\Lambda(\Psi)$ is a section of $\operatorname{End}\left(\underset{2}{\oplus}\left(\operatorname{Ad} P \otimes T^{*} \oplus T^{*}\right)\right)$ whose coefficients are linear combinations of the components of $\Psi$. The section $Q$ of $\oplus\left(\operatorname{Ad} P \otimes T^{*}\right)$ represents the left-hand side of Eq. (C.1.3) minus the term $\left(-\nabla_{A}^{*} \nabla_{A} \Psi\right)$. After contracting Eq. (C.1.14) with $\nabla_{A} \Psi$, one obtains

$$
d^{*} d \frac{\left|\nabla_{A} \Psi\right|^{2}}{2}+\left|\nabla_{A}\left(\nabla_{A} \Psi\right)\right|^{2}-\left(\nabla_{A} \Psi, \Lambda(\Psi) \nabla_{A} \Psi\right)-\left(\nabla_{A} \Psi, \nabla_{A} Q\right)=0
$$

Now multiply Eq. (C.1.16) by $\sigma^{4 \delta}$ to obtain

$$
\begin{aligned}
d^{*} d\left(\sigma^{4 \delta} \frac{\left|\nabla_{A} \Psi\right|^{2}}{2}\right) \leqq & +\sigma^{4 \delta}\left(\nabla_{A} \Psi, \nabla_{A} Q\right)-\sigma^{4 \delta} \frac{1}{2}\left|\nabla_{A}\left(\nabla_{A} \Psi\right)\right|^{2} \\
& +z\left(\sigma^{4 \delta}|\Psi|\left|\nabla_{A} \Psi\right|^{2}+\delta \sigma^{-2+4 \delta}\left|\nabla_{A} \Psi\right|^{2}\right)
\end{aligned}
$$

Let $y \in \mathbb{R}^{3}$. Denote by $\beta^{y}(\cdot)$ the bump function $\beta(|(\cdot)-y|)$. Multiply both sides of Eq. $\left(\right.$ C.1.17) by $\beta^{y}(\cdot)(4 \pi|(\cdot)-y|)$ and then integrate the result over $B^{y}=\left\{x \in \mathbb{R}^{3}:|x-y|<1\right\}$. The result is:

$$
\begin{aligned}
\sigma^{4 \delta} \frac{\left|\nabla_{A} \Psi\right|^{2}}{2}(y) \leqq & -\int_{B^{y}} \beta^{y} \frac{\sigma^{4 \delta}}{|(\cdot)-y|}\left(\nabla_{A} \Psi, \nabla_{A} Q\right)-\frac{1}{2} \int_{B^{y}} \beta^{y} \sigma^{4 \delta} \frac{\left|\nabla_{A}\left(\nabla_{A} \Psi\right)\right|^{2}}{|(\cdot)-y|} \\
& +z \int_{B^{y}} \frac{\beta^{y} \sigma^{4 \delta}}{|(\cdot)-y|}\left(|\Psi|+\delta \sigma^{-2}\right)\left|\nabla_{A} \Psi\right|^{2}+z \int_{B^{y}} \sigma^{4 \delta}\left|\nabla_{A} \Psi\right|^{2}
\end{aligned}
$$


The last term on the right-hand side, above, is due to the fact that

$$
\frac{\left|d^{*} d \beta^{y}\right|}{|(\cdot)-y|}+\frac{2\left|d \beta^{y}\right|}{|(\cdot)-y|^{2}}
$$

are uniformly bounded, independent of $y \in \mathbb{R}^{3}$.

Now, choose $\varrho \in(0,1]$ to be determined shortly. Suppose that $\kappa=\left[\frac{1}{2} \varrho \varepsilon^{2} \zeta\left(\mathfrak{A}^{\delta}(c)\right)^{-1}\right]^{9}$ and that $R=\kappa^{-4 / 9}$ with $\zeta(\cdot)$ as in Eq. (C.1.14). Restrict $y$ to satisfy Eq. (C.1.13). Then

$$
\sigma^{4 \delta}|\Psi|^{2}(y)+\frac{1}{4 \pi} \int \frac{\sigma^{4 \delta}}{|(\cdot)-y|}\left|\nabla_{A} \Psi\right|^{2}<\varrho \varepsilon^{2} .
$$

After writing out the left-hand side of Eq. (C.1.18) and using Eq. (C.1.19), one finds there exists a constant $z<\infty$ which is independent of $y$ satisfying Eq. (C.1.13), $c \in \mathbb{C}^{\delta}$ and $\delta \in\left[0, \frac{1}{4}\right)$ such that

$$
\sigma^{4 \delta}\left|\nabla_{A} \Psi\right|^{2}(y) \leqq z \varrho \varepsilon^{2} .
$$

Assertions (1) and (2) of Lemma C.1.4 follow from Eqs. (C.1.14) and (C.1.20): Choose $\varrho=(z+1)^{-1}$, above, and then $\lambda\left(\mathfrak{H}^{\delta}(c), \varepsilon\right)=\left[\frac{1}{2}(z+1)^{-1} \varepsilon^{2} \zeta\left(\mathfrak{U}^{\delta}(c)\right)^{-1}\right]^{9}$ with $\zeta(\cdot)$ as in Eq. (C.1.14); choose $R\left(\mathfrak{U}^{\delta}(c), \varepsilon\right)=\lambda^{-4 / 9}$.

Proof of Assertion (3) of Lemma C.1.4. Let $w=\frac{1}{2}\left(1-|\Phi|^{2}\right)$. The function $w$ satisfies

$$
d^{*} d w=|g|^{2}
$$

and $w \in L^{6}\left(\mathbb{R}^{3}\right)$. Thus at each $y \in \mathbb{R}^{3}$,

$$
w(y)=\frac{1}{4 \pi} \int \frac{|g|^{2}}{|(\cdot)-y|} .
$$

Suppose that $\varepsilon>0$ is given. For a $\varrho \in(0,1]$ to be determined shortly, let $\lambda^{\prime}\left(\mathfrak{H}^{\delta}(c), \varrho \varepsilon\right)$ and $R^{\prime}\left(\mathfrak{I}^{\delta}(c), \varrho \varepsilon\right)$ be such that Assertions (1) and (2) of Lemma C.1.4 are satisfied. Given $r \in(1, \infty)$, let $y \in \mathbb{R}^{3}$ satisfy Eq. (C.1.13) with $\kappa=\lambda^{\prime}\left(\mathfrak{U}^{\delta}(c), \varrho \varepsilon\right)$ and $R=r+R^{\prime}\left(\mathfrak{U}^{\delta}(c), \varrho \varepsilon\right)$. For such $y$, Eq. (C.1.22) implies

$$
w(y)<\frac{1}{2} \varepsilon^{2} \varrho^{2} r^{2}+\frac{1}{4 \pi} \frac{\mathfrak{U}(c)}{r} .
$$

Take $r=(1+\mathfrak{U}(c)) \varepsilon^{-1}$ and take $\varrho=\varepsilon(1+\mathfrak{U}(c))^{-1}$. Then $w(y)<\varepsilon$. Thus, all assertions of Lemma C.1.4 are obtained if $\lambda=\lambda^{\prime}\left(\mathfrak{U}^{\delta}(c), \varepsilon^{2}(1+\mathfrak{A}(c))^{-1}\right)$ and if $R=R^{\prime}\left(\mathfrak{U}^{\delta}(c), \varepsilon\right)+\varepsilon^{-1}(1+\mathfrak{U}(c))$.

\section{C.2. Exponential Decay Estimates}

To prove Proposition C.1.1, $\delta$-independent estimates for a solution, $c$, of Eq. (C.1.1) are required which allow one to approximate the fields $(f, g)$ in $\mathbb{R}^{3} \backslash U[c](\kappa)$ by solutions there to linear equations. This enables one to obtain, for a suitable $\kappa$, 
multipole expansions for these curvatures in $\mathbb{R}^{3} \backslash U[c](\kappa)$. The first step, Lemma C.2.1 below, asserts that the fields are essentially abelian in $\mathbb{R}^{3} \backslash U[c](\kappa)$.

Let $c=(A, \Phi)$ be a solution to Eq. (C.1.1). Let $\kappa>0$ and let $\left\{U_{j}: j \in\{1, \ldots, n[c](\kappa)\}\right\}$ be the path components of $U[c](\kappa)$. To each $U_{j}$, associate its center of mass

$$
x_{j}=\left(\int_{U_{j}} x d^{3} x\right)\left(\int_{U_{j}} d^{3} x\right)^{-1} .
$$

Lemma C.2.1. There exists $\delta_{0} \in\left[0, \frac{1}{4}\right)$ and continuous functions $\kappa_{0}:[0, \infty) \rightarrow(0,1]$ and $m:[0, \infty) \rightarrow[0, \infty)$ with the following significance: Let $\delta \in\left[0, \delta_{0}\right)$ and let $c=(A, \Phi) \in \mathfrak{C}^{\delta}$ be a solution to Eq. (C.1.1). Let $\kappa=\kappa_{0}\left(\mathfrak{H}^{\delta}(c)\right)$. Let $\left\{U_{j}\right\}$ be the path components of $U[c](\kappa)$ and define $\left\{x_{j}\right\} \subset \mathbb{R}^{3}$ by Eq. (C.2.1). If $x \in \mathbb{R}^{3}$, then

$$
\sigma^{\delta}(x)\left|\left[\Phi, F_{A}\right]\right|(x)+\left|\left[\Phi, D_{A} \Phi\right]\right|(x) \leqq m\left(\mathfrak{H}^{\delta}(c)\right) \sum_{j}\left(1+\left|x-x_{j}\right|\right)^{-5 / 2} .
$$

The remainder of this section is occupied with the proof of Lemma C.2.1.

Proof of Lemma C.2.1. Let $\Psi^{\prime}=\sigma^{2 \delta}[\Phi, \Psi]$ with $\Psi=(f, g)$ as in Sect. C.1. The idea is to derive an equation for $\Psi^{\prime}$ from Eq. (C.1.3) which shows that for suitable $\kappa_{0}\left(\mathfrak{I}^{\delta}(c)\right)$ and $R\left(\mathfrak{H}^{\delta}(c)\right), \Psi^{\prime}$ satisfies the following inequality at $x \in \mathbb{R}^{3}$ with $\operatorname{dist}\left(x, U[c]\left(\kappa_{0}\right)\right)>R$ :

$$
d^{*} d\left|\Psi^{\prime}\right|^{2}+\sigma^{-2 \delta}\left|\Psi^{\prime}\right|^{2}<0 .
$$

Then Lemma C.2.1 is obtained by applying the comparison principle to Eq. (C.2.2) with a suitable choice of comparison function.

To derive Eq. (C.2.2), take the commutator of Eq. (C.1.3) with $\Phi$ and multiply the result by $\sigma^{2 \delta}$. After commuting through covariant derivatives, one obtains the following equation for $\Psi^{\prime}$ :

$$
\begin{aligned}
-\nabla_{A}^{*} \nabla_{A} \Psi^{\prime} & +\frac{4 \delta}{\sigma^{2}}\left(\mathbf{x}, \nabla_{A}\right) \Psi^{\prime}+\frac{\delta}{\sigma^{2}} * D_{A} * \mathbf{x} \wedge\left(\begin{array}{c}
{[\Phi, f]} \\
0
\end{array}\right)+\sigma^{2 \delta}\left[\Psi, \nabla_{A} \Psi\right]_{1} \\
& +\sigma^{2 \delta}[\Psi, \Psi]_{2}+\sigma^{-2 \delta}\left[\Phi,\left[\Phi, \Psi^{\prime}\right]\right]+\delta \sigma^{-2 \delta} \Lambda\left(\Psi^{\prime}\right)=0 .
\end{aligned}
$$

Here, $[\cdot, \cdot]_{1}$ is a particular extension of the commutator pairing $[\cdot, \cdot]: \bigoplus_{2} \operatorname{Ad} P$ $\rightarrow \operatorname{Ad} P$ to a bilinear pairing from $\left(\underset{2}{\oplus} \operatorname{Ad} P \otimes T^{*} \oplus T^{*}\right) \oplus\left(\underset{2}{\oplus} \operatorname{Ad} P \otimes T^{*}\right)$ to $\oplus \underset{2}{\oplus} \operatorname{Ad} P \otimes T^{*}$. This means that if one considers $\nabla_{A} \Psi$ and $\Psi$ as $\operatorname{Ad} P$ valued tensors on $\mathbb{R}^{3}$, then $\left[\Psi, \nabla_{A} \Psi\right]_{1}$ is an $\operatorname{Ad} P$ valued tensor whose components are linear combinations of the commutators of the components of $\nabla_{A} \Psi$ and $\Psi$. The coefficients of these linear combinations are fixed real numbers. Likewise, $[\Psi, \Psi]_{2}$ is an $\operatorname{Ad} P$ valued tensor on $\mathbb{R}^{3}$ whose components are linear combinations of commutators of components of $\Psi$ with each other. The coefficients of these terms are $x$-dependent, but they are bounded independently of $x$ by a fixed $z<\infty$ which is independent of $c \in \mathbb{C}$ and $\delta$. In Eq. (C.2.3), $\Lambda$ is an $x$-dependent section of End $\left(\underset{2}{\oplus} \operatorname{Ad} P \otimes T^{*}\right)$ which satisfies an $x, c \in \mathbb{C}^{\delta}$ and $\delta$ independent bound. 
To continue, contract both sides of Eq. (C.2.3) with $\Psi^{\prime}$ to obtain

$$
\begin{aligned}
d^{*} d \frac{\left|\Psi^{\prime}\right|^{2}}{2} & +\left|\nabla_{A} \Psi^{\prime}\right|^{2}+\sigma^{-2 \delta}\left|\left[\Phi, \Psi^{\prime}\right]\right|^{2} \leqq z \delta \sigma^{-2 \delta}\left(\left|\Psi^{\prime}\right|^{2}+\left|\Psi^{\prime}\right|\left|\nabla_{A} \Psi^{\prime}\right|\right) \\
& +\left|\left(\Psi^{\prime}, \sigma^{2 \delta}\left[\Psi, \nabla_{A} \Psi\right]_{2}\right)\right|+\left|\left(\Psi^{\prime}, \sigma^{2 \delta}[\Psi, \Psi]_{1}\right)\right| .
\end{aligned}
$$

In Eq. (C.2.4), $z<\infty$ is a constant which is independent $x, c \in \mathfrak{C}^{\delta}$ and $\delta \in\left[0, \frac{1}{4}\right)$.

Let $\kappa=\lambda\left(\mathfrak{H}^{\delta}(c), \frac{1}{8}\right)$ and $R=R\left(\mathfrak{H}^{\delta}(c), \frac{1}{8}\right)$ with $\lambda$ and $R$ as specified in Lemma C.1.4. Since the diameter of $U[c](\kappa)$ is less than $2 \mathfrak{A}(c) / \kappa$, Lemma C.1.4 implies that the set

$$
V[c](\kappa)=\left\{x \in \mathbb{R}^{3}:\left|x-x_{j}\right|>R+4 \mathfrak{A}(c) \kappa^{-1} \quad \text { for all } j=1, \ldots, n[c](\kappa)\right\}
$$

has the property that

$$
|\Phi|>\frac{7}{8} \quad \text { on } \quad V[c](\kappa) .
$$

Further, the following simple identities hold on $V[c](\kappa)$ :

(1) $\Psi^{\prime}=|\Phi|^{-2}\left[\Phi,\left[\Psi^{\prime}, \Phi\right]\right]$,

(2) $\sigma^{2 \delta}\left[\Phi, \nabla_{A} \Psi\right]=\nabla_{A} \Psi^{\prime}-\sigma^{2 \delta}[g, \Psi]-2 \delta \sigma^{-2} \mathbf{x} \Psi^{\prime}$,

(3) $\left|\sigma^{2 \delta}[\Psi, \Psi]_{2}\right| \leqq z\left|\Psi^{\prime}\right||\Psi|$,

(4) $\left|\Psi^{\prime}\right|<\frac{1}{8}$.

From Eqs. (C.2.6-7) one derives readily that the left-hand side of Eq. (C.2.4) is bounded on $V[c](\kappa)$ by

$$
z \delta \sigma^{-2 \delta}\left(\left|\Psi^{\prime}\right|^{2}+\left|\nabla_{A} \Psi^{\prime}\right|^{2}\right)+z|\Psi|\left(\left|\Psi^{\prime}\right|^{2}+\left|\Psi^{\prime}\right|\left|\nabla_{A} \Psi^{\prime}\right|\right)+z\left|\nabla_{A} \Psi\right|\left|\Psi^{\prime}\right|^{2} .
$$

In Eq. (C.2.8), the constant $z<\infty$ is independent of $c \in \mathbb{C}^{\delta}$ and $\delta \in\left[0, \frac{1}{4}\right)$. Equations (C.2.6-8) together with Eq. (C.2.4) imply that on $V[c](\kappa)$,

$$
d^{*} d\left|\Psi^{\prime}\right|^{2}+\sigma^{-2 \delta}\left(\frac{3}{2}-z\left(\delta+\sigma^{2 \delta}|\Psi|+\sigma^{2 \delta}\left|\nabla_{A} \Psi\right|\right)\left|\Psi^{\prime}\right|^{2} \leqq 0 .\right.
$$

Here, $z<\infty$ is a constant which is independent of $c \in \mathbb{C}^{\delta}$ and $\delta \in\left[0, \frac{1}{4}\right)$.

Choose $\delta_{1}=16^{-1}(1+z)^{-1}$. Choose $\kappa_{0}\left(\mathfrak{I}^{\delta}(c)\right)$ to be $\lambda\left(\mathfrak{H}^{\delta}(c),(1+16 z)^{-1}\right)$ with $\lambda$ given by Lemma C.1.4. Let $R=R\left(\mathfrak{H}^{\delta}(c), 16^{-1}(1+z)^{-1}\right)$ as given by Lemma C.1.4. If dist $\left(x, U[c]\left(\kappa_{0}\right)\right)>R$, then Lemma C.1.4 and Eq. (C.2.9) imply that Eq. (C.2.2) holds with $\delta \in\left[0, \delta_{1}\right]$.

Equation (C.2.2) is an inequality for which the comparison principle was designed. For $\delta \in\left[0, \delta_{1}\right]$, consider the function

$$
u_{j}(x)=\frac{1}{4 \pi} \frac{1}{\left|x-x_{j}\right|} \exp \left\{-\frac{1}{2}\left(1+|x|^{2}+\left|x-x_{j}\right|^{2}\right)^{-\delta / 2}\left|x-x_{j}\right|\right\} .
$$

By using the fact that

$$
\begin{aligned}
\left|d\left(1+|x|^{2}+\left|x-x_{j}\right|^{2}\right)^{-\delta / 2}\right| & \leqq \delta\left(1+|x|^{2}+\left|x-x_{j}\right|^{2}\right)^{-\delta / 2}\left|x-x_{j}\right|^{-1}, \\
\left|d^{*} d\left(1+|x|^{2}+\left|x-x_{j}\right|^{2}\right)^{-\delta / 2}\right| & \leqq 6 \delta\left(1+|x|^{2}+\left|x-x_{j}\right|^{2}\right)^{-\delta / 2}\left|x-x_{j}\right|^{-2},
\end{aligned}
$$


and that $\sigma(x) \leqq\left(1+|x|^{2}+\left|x-x_{j}\right|^{2}\right)$, one concludes that there exists $\delta_{0} \in\left(0, \delta_{1}\right]$ such that for all $\delta \leqq \delta_{0}$, and for all $x \in \mathbb{R}^{3} \backslash\left\{x_{j}\right\}$,

$$
-d^{*} d u_{j}-\sigma^{-2 \delta} u_{j} \leqq 0 \text {. }
$$

Using Lemma C.1.4 and the comparison principle, one obtains for all $\delta \in\left[0, \delta_{0}\right)$ and $x \in \mathbb{R}^{3}$ with $\operatorname{dist}\left(x, U[c]\left(\kappa_{0}\right)\right) \geqq R$ the bound

$$
\left|\Psi^{\prime}\right|^{2}(x) \leqq \zeta\left(\mathfrak{Q}^{\delta}(c)\right) \sum_{j} u_{j}
$$

Here, $\zeta(\cdot)$ is a continuous function on $[0, \infty)$ which is independent of $c \in \mathbb{C}^{\delta}$ and $\delta$. Thus, one concludes that Lemma C.2.1 follows from Eq. (C.2.12) and

Lemma C.2.2. Define $u_{j}$ by Eq. (C.2.10). Then $\left(\sigma^{-4 \delta} u_{j}\right)(x) \leqq z\left|x-x_{j}\right|^{-5}$, where $z<\infty$ is independent of $x$ and $x_{j}$ in $\mathbb{R}^{3}$.

Proof of Lemma C.2.2. Observe first that there exists $z<\infty$ such that when $t \geqq 0, \quad \exp \left(-\frac{1}{4} t^{1 / 9}\right) \leqq z(1+t)^{-5}$. Therefore, if $\left|x-x_{j}\right| \geqq\left(1+|x|+\left|x-x_{j}\right|\right)^{9 \delta / 8}$, then $\quad \sigma^{-4 \delta}(x) u_{j}(x) \leqq \sigma^{-4 \delta}(x)\left(1+\left|x-x_{j}\right|\right)^{-5}$. However, if $2<\left|x-x_{j}\right|$ $<\left(1+|x|+\left|x-x_{j}\right|\right)^{9 \delta / 8}$, then

$$
\sigma^{-4 \delta} u_{j} \leqq z \sigma^{-4 \delta}\left|x-x_{j}\right|^{-1}\left(1+\sigma^{-\delta}\left(x-x_{j} \mid\right)^{-4} \leqq z\left(1+\left|x-x_{j}\right|\right)^{-5} .\right.
$$

\section{C.3. Power Law Estimates for $\Phi$}

The uniform estimates provided by Lemma C.2.1 indicate that the fields in $\mathbb{R}^{3} \backslash U[c](\kappa)$ for a suitable $\kappa$ are almost abelian if $c \in \mathbb{C}^{\delta}$ satisfies Eq. (C.1.1) and if $\delta$ is sufficiently small. In the present section, it will be proved that the field $|\Phi|$ looks like a solution to Laplace's equation on $\mathbb{R}^{3} \backslash U[c](\kappa)$. The precise statement is

Lemma C.3.1. There exists $m_{1} \in C^{0}([0, \infty) ;[0, \infty))$ with the following significance: Let $\delta_{0}$ and $\kappa_{0}$ be as in Lemma C.2.1. For $\delta \in\left[0, \delta_{0}\right)$, let $c=(A, \Phi) \in \mathfrak{C}^{\delta}$ be a solution to Eq. (C.1.1). For $\kappa=\kappa_{0}\left(\mathfrak{H}^{\delta}(c)\right)$, let $\left\{U_{j}\right\}$ be the path components of $U[c](\kappa)$. Define $\left\{x_{j}\right\}$ by Eq. (C.2.2). There exist real numbers $\left\{\mathfrak{a}_{j}: j=1, \ldots, n[c](\kappa)\right\}$, with each $\left|\mathfrak{a}_{j}\right|<m_{1}\left(\mathfrak{U}^{\delta}(c)\right)$, such that if $x \in \mathbb{R}^{3}$, then

(1) ||$\Phi\left|(x)-1+\sum_{j} \mathfrak{a}_{j}\right| x-\left.x_{j}\right|^{-1}\left|\leqq m_{1}\left(\mathfrak{U}^{\delta}(c)\right) \sum_{j}\right| x-\left.x_{j}\right|^{-2}$,

(2) $\left|\left(\Phi, D_{A} \Phi\right)(x)+\sum_{j} \mathfrak{a}_{j} d\right| x-\left.x_{j}\right|^{-1}\left|\leqq m_{1}\left(\mathfrak{U}^{\delta}(c)\right) \sum_{j}\right| x-\left.x_{j}\right|^{-3}$.

The numbers $\left\{\mathfrak{a}_{j}\right\}$ will be identified in Sect. D.2. The remainder of this section is occupied by the proof of Lemma C.3.1.

Proof of Assertion (1) of Lemma C.3.1. Let $\lambda\left(\mathfrak{H}^{\delta}(c), \frac{1}{8}\right)$ and $R\left(\mathfrak{U}^{\delta}(c), \frac{1}{8}\right)$ be given by Lemma C.1.4. On the set

$$
V=\left\{x \in \mathbb{R}^{3}: \operatorname{dist}\left(x ; U[c](\kappa) \cup U[c]\left(\lambda\left(\mathfrak{U}^{\delta}(c), \frac{1}{8}\right)\right)\right)>R\left(\mathfrak{I}^{\delta}(c), \frac{1}{8}\right)+1\right\} .
$$


Then $|\Phi|(x)>\frac{7}{8}$ and $1-|\Phi|$ satisfies the equation

$$
d^{*} d(1-|\Phi|)=|\Phi|^{-1}\left|\left[\Phi, D_{A} \Phi\right]\right|^{2} .
$$

Let $\eta \in C_{0}^{\infty}\left(\mathbb{R}^{3} ;[0,1]\right)$ be a function which is identically 1 on the set $V$, identically 0 on the set $\left\{x \in \mathbb{R}^{3}: \operatorname{dist}(x, V)>1\right\}$, and such that $|d \eta|+\left|d^{*} d \eta\right|<4$. Then $\eta(1-|\Phi|)$ satisfies at $x \in \mathbb{R}^{3}$

$$
(1-|\Phi|) \eta(x)=\frac{1}{4 \pi} \int \frac{1}{|(\cdot)-x|}\left\{\eta|\Phi|^{-1}\left|\left[\Phi, D_{A} \Phi\right]\right|^{2}+(1-|\Phi|) d^{*} d \eta+2(d|\Phi|, d \eta)\right\} .
$$

A multipole expansion for $1-|\Phi|$ is to be generated from Eq. (C.3.3). For this purpose, let $G$ denote the term in brackets in the integrand of Eq. (C.3.3). A consequence of Lemmas C.1.3 and C.2.1 is that there exists a continuous function $\zeta(\cdot):[0, \infty) \rightarrow[0, \infty)$ which is independent of $c \in \mathbb{C}^{\delta}$ and $\delta \in\left[0, \delta_{0}\right)$ such that for all $x \in \operatorname{supp} \eta$,

$$
|G(x)| \leqq \zeta\left(\mathfrak{A}^{\delta}(c)\right) \sum_{j}\left(1+\left|x-x_{j}\right|\right)^{-5} .
$$

It is convenient to let $\psi_{j}=\left(1+\left|x-x_{j}\right|\right)^{-5}$ and set

$$
G_{j}=\left(\sum_{k} \psi_{k}\right)^{-1} \psi_{j} G
$$

Observe that Eqs. (C.3.3-4) imply that

$$
\left|(1-|\Phi|) \eta(x)-\frac{1}{4 \pi} \sum_{j} \frac{1}{\left|x-x_{j}\right|} \int_{\mathbb{R}^{3}} G_{j}\right| \leqq \frac{1}{4 \pi} \sum_{j}\left|\int_{\mathbb{R}^{3}} G_{j}\left(\frac{1}{|x-(\cdot)|}-\frac{1}{\left|x-x_{j}\right|}\right)\right| .
$$

The proof of Assertion (1) of Lemma C.3.1 requires a proof that $a_{j}=\int_{\mathbb{R}^{3}} G_{j}$ is bounded by some $m_{1}\left(\mathfrak{H}^{\delta}(c)\right)$, and an estimate for the right-hand side of Eq. (C.3.5) which establishes that

$$
\left|\int_{\mathbb{R}^{3}} G_{j}\left(\frac{1}{|x-(\cdot)|}-\frac{1}{\left|x-x_{j}\right|}\right)\right| \leqq z \cdot \zeta\left(\mathfrak{A}^{\delta}(c)\right)\left|x-x_{j}\right|^{-2},
$$

with $z<\infty$ a constant which is independent of $c \in \mathbb{C}^{\delta}$ and $\delta \in\left[0, \delta_{0}\right)$. For $\mathfrak{a}_{j}$, note that Eq. (C.3.4) implies the inequality

$$
\int_{\mathbb{R}^{3}} G_{j} \leqq \zeta\left(\mathfrak{U}^{\delta}(c)\right) \int_{\mathbb{R}^{3}} d^{3} x\left(1+\left|x-x_{j}\right|\right)^{-5} \leqq 2 \pi \zeta\left(\mathfrak{A}^{\delta}(c)\right) .
$$

To prove that Eq. (C.3.6) holds, write

$$
\frac{1}{|x-y|}-\frac{1}{\left|x-x_{j}\right|}=\int_{0}^{1} d t \frac{d}{d t} \frac{1}{\left|x-x_{j}-t\left(y-x_{j}\right)\right|}
$$

This allows the left-hand side of Eq. (C.3.6) to be bounded by

$$
(4 \pi)^{-1} \int_{0}^{1} d t \int d^{3} y\left|G_{j}(y)\right|\left|y-x_{j}\right|\left|x-x_{j}-t\left(y-x_{j}\right)\right|^{-2} .
$$


To complete the proof of Assertion (1) of Lemma C.3.1, break the integral in Eq. (C.3.8) into two parts, $I_{1}$ and $I_{2}$. Here $I_{1}$ is the integral over $L=\left\{y \in \mathbb{R}^{3}:\left|x-x_{j}-t\left(y-x_{j}\right)\right|>\frac{1}{2}\left|x-x_{j}\right|\right\}$, and $I_{2}$ is the integral over $\mathbb{R}^{3} \backslash L$. For $I_{1}$, observe that Eq. (C.3.4) implies the bound

$$
I_{1} \leqq \frac{1}{\pi} \frac{1}{\left|x-x_{j}\right|^{2}} \int_{\mathbb{R}^{3}}\left|G_{j}(y)\right|\left|y-x_{j}\right| \leqq 4 \cdot \zeta\left(\mathfrak{A}^{\delta}(c)\right)\left|x-x_{j}\right|^{-2} .
$$

For $I_{2}$, note that on $\mathbb{R}^{3}|L| y-,x_{j}\left|\geqq t^{-1} \frac{1}{2}\right| x-x_{j} \mid$, so Eqs. $($ C.3.4, 8) imply that

$$
I_{2} \leqq z \zeta\left(\mathfrak{I}^{\delta}(c)\right) \int_{0}^{1} t d t\left|x-x_{j}\right|\left(t+\frac{1}{2}\left|x-x_{j}\right|\right)^{-4} \leqq z \zeta\left(\mathfrak{U}^{\delta}(c)\right)\left|x-x_{j}\right|^{-3} .
$$

Proof of Assertion (2) of Lemma C.3.1. Since $d|\Phi|=|\Phi|^{-1}\left(\Phi, D_{A} \Phi\right)$, this assertion follows by establishing that the expansion provided by Assertion (1) is differentiable. From Eq. (C.3.3) one derives

$$
\left|d(|\Phi| \eta)+\frac{1}{4 \pi} \sum_{j} d\right| x-\left.x_{j}\right|^{-1} \int_{\mathbb{R}^{3}} G_{j}\left|\leqq \frac{1}{4 \pi} \sum_{j} \int_{\mathbb{R}^{3}} d^{3} y\right| G_{j}|| d|x-y|^{-1}-d\left|x-x_{j}\right|^{-1} \mid .
$$

The assertion then follows by establishing that

$$
\int d y\left|G_{j}\right|\left|\frac{(\mathbf{x}-\mathbf{y})}{|x-y|^{3}}-\frac{\left(\mathbf{x}-\mathbf{x}_{j}\right)}{\left|x-x_{j}\right|^{3}}\right| \leqq z \cdot \zeta\left(\mathfrak{l}^{\delta}(c)\right) \cdot\left|x-x_{j}\right|^{-3},
$$

with $z<\infty$, a constant independent of $c \in \mathfrak{C}^{\delta}$ and $\delta \in\left[0, \delta_{0}\right)$.

To obtain Eq. (C.3.10), it is convenient to calculate the bound

$$
\int d^{3} y\left|\psi_{j}\right|\left|y-x_{j}\right||y-x|^{-1} \leqq z\left|x-x_{j}\right|^{-1},
$$

with $z<\infty$, a constant independent of $x$ and $x_{j}$. To establish Eq. (C.3.10) one also needs the last inequality, below:

$$
\begin{aligned}
\left|\frac{\mathbf{x}-\mathbf{y}}{|x-y|^{3}}-\frac{\mathbf{x}}{|x|^{3}}\right| & \leqq\left|\frac{\mathbf{x}-\mathbf{y}}{|x-y|^{3}}-\frac{\mathbf{x}}{|x|^{2}|x-y|}\right|+\frac{1}{|x|}\left|\frac{1}{|x-y|}-\frac{1}{|x|}\right|, \\
& \leqq \frac{1}{|x|}\left|\frac{1}{|x-y|}-\frac{1}{|x|}\right|+\frac{|y|}{|x|^{2}} \frac{1}{|x-y|}+\left|\frac{1}{|x-y|^{2}}-\frac{1}{|x|^{2}}\right|, \\
& \leqq \frac{2}{|x|}\left|\frac{1}{|x-y|}-\frac{1}{|x|}\right|+\frac{|y|}{|x|^{2}} \frac{1}{|x-y|}+\frac{1}{|x-y|}\left|\frac{1}{|x-y|}-\frac{1}{|x|}\right| .
\end{aligned}
$$

With Eqs. $($ C.3.4, 6, 11-12) one immediately finds that the left-hand side of Eq. (C.3.10) is bounded by

$$
z \zeta\left(\mathfrak{A}^{\delta}(c)\right)\left\{\left|x-x_{j}\right|^{-3}+\int_{\mathbb{R}^{3}} d^{3} y \psi_{j}(y) \frac{1}{|x-y|}\left|\frac{1}{|x-y|}-\frac{1}{\left|x-x_{j}\right|}\right|\right\} .
$$

The last term in Eq. (C.3.13) is evaluated using Eq. (C.3.7), and one obtains the bound

$$
\int_{0}^{1} d t \int_{\mathbb{R}^{3}} d^{3} y \psi_{j}(y)\left|y-x_{j}\right| \frac{1}{|x-y|} \frac{1}{\left|x-x_{j}-t\left(y-x_{j}\right)\right|^{2}} .
$$


The evaluation of Eq. (C.3.14) proceeds as before, by breaking the integral into $I_{1}$ and $I_{2}$ with $I_{1}$ the integral over $L$ and with $I_{2}$ the integral over $\mathbb{R}^{3} \backslash L$. For $I_{1}$, one obtains with Eq. (C.3.11) that $I_{1} \leqq z\left|x-x_{j}\right|^{-3}$. As for $I_{2}$, one obtains

$$
\begin{aligned}
I_{2} & \leqq z\left|x-x_{j}\right|^{-4} \int_{0}^{1} d t t^{2} \int_{0}^{1} d r(r(1-t))^{-1} \min (r,(1-t)), \\
& \leqq z\left|x-x_{j}\right|^{-4} .
\end{aligned}
$$

\section{C.4 Power Law Estimates for $\boldsymbol{A}$}

The purpose of this section is to prove that if $c=(A, \Phi) \in \mathbb{C}^{\delta}$ is a solution to Eq. (C.1.1), then for $\kappa=\kappa_{0}\left(\mathfrak{U}^{\delta}\right)$, the real 2-form $\left(\Phi, F_{A}\right)$ on $\mathbb{R}^{3} \backslash U[c](\kappa)$ is approximately the curvature 2 -form for an abelian, Dirac monopole connection [21]. The precise statement is

Lemma C.4.1. Let $\delta_{0}, \kappa_{0}$ be as in Lemma C.2.1. There exists $\delta_{1} \in\left(0, \delta_{0}\right)$ and a continuous function $m_{2}:[0, \infty) \rightarrow[0, \infty)$ with the properties below: Let $\delta \in\left[0, \delta_{1}\right)$, and let $c \in \mathfrak{C}^{\delta}$ be a solution to Eq. (C.1.1). Let $\kappa=\kappa_{0}\left(\mathfrak{U}^{\delta}(c)\right)$ and let $\left\{x_{j}\right\}$ be as in Lemma C.3.1. There exist real numbers $\left\{\mathrm{n}_{j}: j=1, \ldots, n[c](\kappa)\right\}$ and vectors $\left\{l_{j}, j=1, \ldots, n[c](\kappa)\right\}$ with each $\left|\boldsymbol{n}_{j}\right|,\left|l_{j}\right|<m_{2}\left(\mathfrak{U}^{\delta}(c)\right)$ such that at $x \in \mathbb{R}^{3}$,

$$
\begin{aligned}
& \left|\left(\Phi, F_{A}\right)+\sum_{j} \mathrm{n}_{j} d\right| x-\left.x_{j}\right|^{-1}+\sum_{j} *\left(l_{j} \wedge d\left|x-x_{j}\right|^{-1}\right) \mid \\
& \quad \leqq m_{2}\left(\mathfrak{A}^{\delta}(c)\right) \cdot \sum_{j}\left\{\left|x-x_{j}\right|^{-3}+\delta\left|x-x_{j}\right|^{-2}(1+|x|)^{1 / 32} \cdot \max \left(1,\left(\frac{1+\left|x-x_{j}\right|}{1+|x|}\right)^{1 / 16}\right) .\right.
\end{aligned}
$$

The $\left\{n_{j}, l_{j}\right\}$ are determined in Sect. D.2. This section contains the proof of Lemma C.4.1. The asserted a priori estimates are obtained for a solution $c=(A, \Phi) \in \mathfrak{C}^{\delta}$ to Eq. (C.1.1) by studying the equations for $\left(\Phi, F_{A}\right)$ :

$$
d * \sigma^{2 \delta}\left(\Phi, F_{A}\right)-\sigma^{2 \delta}\left(D_{A} \Phi \wedge * F_{A}\right)=0, \quad d\left(\Phi, F_{A}\right)-\left(D_{A} \Phi \wedge F_{A}\right)=0 .
$$

Proof of Lemma C.4.1. As in the proof of Assertion (1) of Lemma C.3.1, introduce the set $V$, and the bump function $\eta$. For $x \in V,|\Phi|(x)>\frac{7}{8}$, and introduce for such $x$

$$
g^{T}(x)=|\Phi|^{-2}[\Phi,[g, \Phi]], \quad f^{T}(x)=|\Phi|^{-2}[\Phi,[f, \Phi]] .
$$

Define a real 1 -form by

$$
* \tau=\exp (-\eta|\Phi|)\left(\Phi, F_{A}\right)
$$

The 1-form $\tau$ obeys

$$
\begin{aligned}
d \sigma^{2 \delta} \tau & =e^{-\eta|\Phi|} \sigma^{2 \delta}\left\{(1-\eta)(g \wedge f)+\eta\left(g^{T} \wedge f^{T}\right)-|\Phi| d \eta \wedge(\Phi, f)\right\} \\
& \equiv * \sigma^{2 \delta} q \\
d * \tau & =e^{-\eta|\Phi|}\left\{(1-\eta)(g \wedge * f)+\eta\left(g^{T} \wedge * f^{T}\right)-|\Phi| d \eta \wedge *(\Phi, f)\right\} \\
& \equiv * p
\end{aligned}
$$

In order to estimate $\tau$, the Hodge theorem on $\mathbb{R}^{3}$ is required. 
Lemma C.4.2. For $\delta \in\left[0, \delta_{0}\right)$, let $c \in \mathfrak{C}^{\delta}$ be a solution to Eq. (C.1.1). Define the 1-form $\tau$ as in Eq. (C.4.2). There exists a unique $(u, \omega) \in \Gamma\left(\mathbb{R} \oplus T^{*}\right) \cap L^{6}$ with the property that $\left.\nabla(u, \omega) \in L^{2}\left(\mathbb{R} \oplus T^{*}\right) \otimes T^{*}\right), d * \omega=0$ and $\sigma^{2 \delta} \tau=d u+* d \omega$.

Proof of Lemma C.4.2. Since one can show that $\tau \in L^{2}\left(T^{*}\right)$, this is a standard result, cf. [25] and [6, Proposition I.7.6].

The function $u$ satisfies the following equation:

$$
\nabla^{*} \nabla u=p
$$

To obtain estimates from Eq. (C.4.5), introduce for each $j \in\{1, \ldots, n[c](\kappa)\}$ the function $\psi_{j}=\left(1+\left|x-x_{j}\right|\right)^{-5}$. Due to Lemma C.2.1,

$$
|p| \leqq \zeta\left(\mathfrak{H}^{\delta}(c)\right) \sum_{j} \psi_{j}(x),
$$

where $\zeta(\cdot):[0, \infty) \rightarrow[0, \infty)$ is continuous and independent of $c \in \mathfrak{C}^{\delta}$ and $\delta \in\left[0, \delta_{0}\right)$. Let

$$
p_{j}=\left(\sum_{k} \psi_{k}\right)^{-1} \psi_{j} p
$$

Then $u=\sum_{j} u_{j}$, where each $u_{j} \in \Gamma\left(T^{*}\right) \cap L^{6}$ and

$$
\nabla^{*} \nabla u_{j}=p_{j}
$$

Lemma C.4.3. Under the same assumptions that hold for Lemma C.4.1, define $p_{j} b y$ Eqs. $(\mathrm{C} .4 .3,5,7)$. There exists a function $\zeta(\cdot):[0, \infty) \rightarrow[0, \infty)$ which is independent of $c \in \mathfrak{C}^{\delta}$ and $\delta \in\left[0, \delta_{0}\right)$; and there exists a number $n_{j}$ with $\left|n_{j}\right|<\zeta\left(\mathfrak{U}^{\delta}(c)\right)$ such that at $x \in \mathbb{R}^{3}$,

$$
\begin{aligned}
\left|u_{j}+n_{j}\right| x-\left.x_{j}\right|^{-1} \mid & \leqq \zeta\left(\mathfrak{U}^{\delta}(c)\right)\left|x-x_{j}\right|^{-2}, \\
\left|d u_{j}+n_{j} d\right| x-\left.x_{j}\right|^{-1} \mid & \leqq \zeta\left(\mathfrak{U}^{\delta}(c)\right)\left|x-x_{j}\right|^{-3} .
\end{aligned}
$$

Proof of Lemma C.4.3. From Eq. (C.4.7),

$$
u_{j}(x)=\frac{1}{4 \pi} \int d^{3} y|x-y|^{-1} p_{j}(y) .
$$

Now copy the proof of Lemma C.3.1 with $p_{j}$, here, replacing $G_{j}$, there. Use Eq. (C.4.6).

With the function $u$ now estimated, turn attention to the 1-form $\omega$. Equation (C.4.3) implies that

$$
* d \sigma^{2 \delta} * d \omega=\sigma^{2 \delta} q-2 \delta \sigma^{-1+2 \delta} *(d \sigma \wedge u) .
$$

Introduce, for each $j \in\{1, \ldots, n[c](\kappa)\}$, the function $\psi_{j}=\left(1+\left|x-x_{j}\right|\right)^{-5}$, again. Due to Lemma C.2.1,

$$
|q| \leqq \zeta\left(\mathfrak{Q}^{\delta}(c)\right) \sum_{j} \psi_{j}(x),
$$

where $\zeta(\cdot):[0, \infty) \rightarrow[0, \infty)$ is continuous and independent of $c \in \mathbb{C}^{\delta}$ and $\delta \in\left[0, \delta_{0}\right)$. For each $j$, let $q_{j}=\left(\sum_{k} \psi_{k}\right)^{-1} \psi_{j} q$, and let

$$
h_{j}=q_{j}-2 \delta \sigma^{-1} *\left(d \sigma \wedge d u_{j}\right),
$$


where $u_{j}$ is defined and estimated in Lemma C.4.3. Under these circumstances, $\omega=\sum_{j} \omega_{j}$, where each $\omega_{j} \in \Gamma\left(T^{*} \mathbb{R}^{3}\right) \cap L^{6}$ satisfies

$$
\nabla^{*} \nabla \omega_{j}+2 \delta \sigma^{-1} *\left(d \sigma \wedge * d \omega_{j}\right)=h_{j} .
$$
Equation (C.4.13) implies that $v=d \omega_{j} \in \Gamma\left(\hat{2} T^{*} \mathbb{R}^{3}\right) \cap L^{2}$ satisfies the integral
equation

$$
v(x)=\frac{1}{4 \pi} \int d^{3} y\left\{d|x-y|^{-1} \wedge h_{j}-2 \delta d|x-y|^{-1} \wedge *\left(\sigma^{-1} d \sigma \wedge * v\right)\right\} .
$$

Estimates for $v(\cdot)$ will be obtained from Eq. (C.4.14) by using the contraction mapping principle on an appropriate Banach space of $C^{0} 1$-forms on $\mathbb{R}^{3}$. This technique obtains $v$ as a limit of $\left\{v^{\alpha}\right\}_{\alpha}=0$, where

$$
v^{0}(x)=\frac{1}{4 \pi} \int d^{3} y d|x-y|^{-1} \wedge h_{j},
$$

and for $\alpha>0, v^{\alpha}=v^{0}-\delta T\left(v^{\alpha-1}\right)$, with

$$
T(v)(x)=(2 \pi)^{-1} \int d^{3} y d|x-y|^{-1} \wedge *\left(\sigma^{-1} d \sigma \wedge * v\right) .
$$

Consider first $v^{0}$ :

Lemma C.4.4. Under the assumptions of Lemma C.4.1, let $v^{0}$ be defined by Eq. (C.4.15). There exists a continuous function $\zeta(\cdot):[0, \infty) \rightarrow[0, \infty)$ which is independent of $c \in \mathfrak{C}^{\delta}$ and $\delta \in\left[0, \delta_{0}\right)$ with the following significance: let

a vector in $\mathbb{R}^{3}$. Then

$$
l_{j} \equiv(4 \pi)^{-1} \int_{\mathbb{R}^{3}} d^{3} y q_{j},
$$

$$
\left|v^{0}(x)+l_{j} \wedge d\right| x-\left.x_{j}\right|^{-1} \mid \leqq \zeta\left(\mathfrak{I}^{\delta}(c)\right)\left(\left|x-x_{j}\right|^{-3}+\delta\left|x-x_{j}\right|^{-2} \ln \left(1+\left|x-x_{j}\right|\right)\right) .
$$

Proof of Lemma C.4.4. Write $v^{0}=v_{1}^{0}+v_{2}^{0}$, where

$$
v_{1}^{0}=\frac{1}{4 \pi} \int d^{3} y d|x-y|^{-1} \wedge q_{j},
$$

and

$$
v_{2}^{0}=-\frac{\delta}{2 \pi} \int d^{3} y d|x-y|^{-1} \wedge *\left(d \sigma \wedge d u_{j}\right) .
$$

The 1-form $v_{2}^{0}$ is analyzed as was $\left(\Phi, D_{A} \Phi\right)$ in the proof of Assertion (2) of Lemma C.3.1, see Eqs. (C.3.11-14). The proof here is, essentially, word for word the proof there, because $q_{j}$ satisfies the same estimates as does the function $G_{j}$ of Eq. (C.3.4).

For $v_{2}^{0}$, define $r(x)=\sigma^{-1} *\left(d \sigma \wedge d u_{j}\right)$. Because of Lemma C.4.3, $r(x)$ satisfies

$$
|r(x)| \leqq \zeta\left(\mathfrak{H}^{\delta}(c)\right) \sigma^{-1}\left(1+\left|x-x_{j}\right|\right)^{-2} .
$$

Thus,

$$
v_{2}^{0} \leqq \delta \cdot \zeta\left(\mathfrak{H}^{\delta}(c)\right) \int d^{3} y|x-y|^{-2}(1+|y|)^{-1}\left|y-x_{j}\right|^{-2} .
$$


Equation (C.4.17) with Assertion (1) of Lemma C.4.5, below, completes the proof of Lemma C.4.4.

Lemma C.4.5. There exists a constant $z<\infty$ with the following properties: For any two points $x, \xi \in \mathbb{R}^{3}$,

(1) $\int d^{3} y(|y|+1)^{-1}|y-x|^{-2}(|y-\xi|+1)^{-2} \leqq z|x-\xi|^{-2} \ln (|x-\xi|+1)$.

(2) For any $\varepsilon \in\left(0, \frac{1}{4}\right)$, let

$$
\eta_{\varepsilon}(y ; \xi)=(1+|y-\xi|)^{-2}(1+|y|)^{\varepsilon} \cdot \max \left(1,(1+|y|)^{-2 \varepsilon}(1+|y-\xi|)^{2 \varepsilon}\right) .
$$

Then

$$
\int d^{3} y|y-x|^{-2}(1+|y|)^{-1} \eta_{\varepsilon}(y ; \xi) \leqq z \varepsilon^{-1} \eta_{\varepsilon}(x ; \xi) .
$$

(3) $(|y-\xi|+1)^{2-\varepsilon} \leqq \eta_{\varepsilon}(y ; \xi)$.

The proof of Lemma C.4.5 is deferred to the end of this section.

Now turn to the sequence $\left\{v^{\alpha}\right\}$ as defined by Eq. (C.4.16). To discuss its convergence, define the Banach space $\mathfrak{L}=\mathfrak{L}\left(x_{j}\right)$ to be the completion of the space

$$
\mathfrak{L}_{0}=\left\{z \eta\left(x ; x_{j}\right)+\phi: z \in \mathbb{R}, \phi \in \Gamma_{0}\left(\hat{\imath}^{*} T^{*}\right) \text { and } \eta=\eta_{\varepsilon} \text { for } \varepsilon=\frac{1}{32}\right\}
$$

with the norm

$$
\|b\|=\left\|\eta^{-1}\left(\cdot ; x_{j}\right) b(\cdot)\right\|_{\infty} .
$$

The relevant properties of $\mathfrak{L}$ are summarized by the following two lemmas.

Lemma C.4.6. The space of sections $\mathfrak{L}$ embeds continuously in $\underset{3}{\times} \overline{C^{0}}\left(\mathbb{R}^{3}\right)$. If $b \in \mathfrak{Q}$, then for some $z \in \mathbb{R}, b=z \eta\left(x ; x_{j}\right)+o\left(\eta\left(x ; x_{j}\right)\right)$.

Lemma C.4.7. There exists $\delta_{1}>0$ such that if $\delta \in\left[0, \delta_{1}\right)$, then

(1) There exists a unique $v \in \mathfrak{L}$ satisfying Eq. (C.4.15).

(2) There exists $\zeta \in C^{0}([0, \infty),[0, \infty))$, which is independent of $c \in \mathbb{C}^{\delta}$ and $\delta \in\left[0, \delta_{1}\right)$ such that if $v^{0} \in \mathfrak{L}$ is a solution to Eq. (C.4.15), then $\left\|v-v_{0}\right\| \leqq \delta \zeta\left(\mathfrak{U}^{\delta}(c)\right)$.

Observe that Lemma C.4.1 is a direct corollary of Lemma C.3.1, Lemmas C.4.3-7, and the next lemma which asserts that Eq. (C.4.3) has a unique $L^{2}$ solution.

Lemma C.4.8. There exists $\delta_{1} \in\left(0, \frac{1}{2}\right]$ such that if $\delta \in\left[0, \delta_{1}\right)$ and $\omega \in \Gamma\left(T^{*} \mathbb{R}^{3}\right) \cap L^{6}$ satisfies $\nabla^{*} \nabla \omega+2 \delta \sigma^{-1} *(d \sigma \wedge * d \omega)=0$, then $\omega \equiv 0$.

Proof of Lemma C.4.6. The embedding of $\mathfrak{L}$ in $\underset{3}{\times C^{0}}\left(\mathbb{R}^{3}\right)$ is clear since $\|\cdot\|$ is stronger than $\|\cdot\|_{C^{0}}$. To prove the second assertion, let $\left\{b_{\alpha}=z_{\alpha} \eta(x, \xi)+\phi_{\alpha}\right\} \in \mathfrak{L}_{0}$ converge in $\mathfrak{L}$ to $b$ with each $z_{\alpha} \in \mathbb{R}$ and $\phi_{\alpha} \in \Gamma_{0}\left(T^{*}\right)$. Convergence with respect to $\|\cdot\|$ implies convergence of $\left\{z_{\alpha}\right\} \in \mathbb{R}$ to some $z \in \mathbb{R}$. Then

$$
\varlimsup_{|x| \rightarrow \infty} \eta(x ; \xi)^{-1}|b-z \eta(x ; \xi)|=0,
$$

as otherwise $\left\|b_{\alpha}-b\right\|$ would not converge to zero as $\alpha \rightarrow \infty$. 
Proof of Lemma C.4.7. Assertion (1) follows immediately from Lemmas C.4.4, 6 and Eqs. (C.4.15-16) if one can establish that there exists $\delta^{\prime}>0$ such that for all $\delta \in\left[0, \delta^{\prime}\right)$, the mapping $\delta T$ as defined in Eq. (C.4.16) induces a contraction mapping from $\mathfrak{L}$ to itself with norm independent of $x_{j}$. Since $T$ itself is independent of $\delta$, such will be the case if one can prove that $T$ defines a bounded linear operator on $\mathfrak{Q}$ with operator norm independent of $x_{j} \in \mathbb{R}^{3}$. This property of $T$ follows from Assertion (2) of Lemma C.4.5 since

$$
|T b(x)| \leqq z\|b\| \int_{\mathbb{R}^{3}} d^{3} y|x-y|^{-2}(1+|y|)^{-1} \eta\left(y ; x_{j}\right) .
$$

Now turn to the proof of Assertion (2) of Lemma C.4.7. For $\delta \in\left[0, \delta^{\prime}\right)$, let $v \in \mathfrak{L}$ be the solution to Eq. (C.4.16) that Assertion (1) provides. If $v_{0}$ is the solution to Eq. (C.4.15), then

$$
v=v_{0}+\delta T(v) .
$$

As $T: \mathfrak{Q} \rightarrow \mathfrak{L}$ has norm bounded independently of $x_{j}$, Eq. (C.4.20) implies that $\delta_{1} \in\left(0, \delta^{\prime}\right)$ exists such that for all $\delta \in\left[0, \delta_{1}\right),\|v\| \leqq 2\left\|v_{0}\right\|$. Now Lemma C.4.4, Assertion (3) of Lemma C.4.5 and Eq. (C.4.20) immediately give the result.

Proof of Lemma C.4.8. A standard argument with cut-offs puts $\nabla \omega \in L^{2}\left(T^{*} \mathbb{R}^{3}\right)$, where it satisfies $\|\nabla \omega\|_{2} \leqq 4 \delta\left\|\sigma^{-1} \omega\right\|_{2}$. Lemma A.4.1 of [9] finishes the proof.

This section ends with the

Proof of Lemma C.4.5. For Assertion (1), one may first assume that $r=|x-\xi|>1$. Then

$$
(1) \leqq \int_{\mathbb{R}^{3}} d^{3} y|x-y|^{-2}|\xi-y|^{-2}(1+|y|)^{-1} .
$$

Break the integral above into two parts, $I_{1}=\left\{y:|y|>\frac{1}{2}|y-\xi|\right.$ or $\left.\frac{1}{2}|y-x|\right\}$ and $I_{2}$ is the remainder. For $I_{1}$, assume $|y|>\frac{1}{2}|y-x|$, whence by changing $y \rightarrow y+x$,

$$
I_{1} \leqq \int_{\mathbb{R}^{3}} d^{3} y|y|^{-2}(1+|y|)^{-1}|y-\xi+x|^{-2} .
$$

After rescaling, $y \rightarrow r y$, one has

$$
I_{1} \leqq r^{-2} \int_{\mathbb{R}^{3}} d^{3} y|y|^{-2}\left(r^{-1}+|y|\right)^{-1}|y-\hat{n}|^{-2} \leqq z r^{-2} \ln r .
$$

Here $\hat{n}=(\xi-x) r^{-1}$ is a unit vector. For $I_{2}$, note that at least one of $|y-\xi|$ or $|y-x| \geqq \frac{1}{2} r$ (by the triangle inequality), and hence

$$
I_{2} \leqq z \int_{\mathbb{R}^{3}} d^{3} y(r+|y|)^{-2}(1+|y|)^{-1}|y|^{-2} \leqq z r^{-2} \ln r .
$$

For Assertion (2), the left-hand integral has two parts, $I_{1}=\{y:|y|>|y-\xi|\}$ and $I_{2}$, the remainder. For $I_{1}$, one has

$$
I_{1} \leqq \int_{|y|>|y-\xi|} d^{3} y|x-y|^{-2}(1+|y|)^{-1+\varepsilon}|y-\xi|^{-2} .
$$

Now, change $y \leadsto y+x$ and since $(1+|y|)^{-1}<|y-\xi|^{-1}$ on the domain of integration,

$$
I_{1} \leqq \int_{\mathbb{R}^{3}} d^{3} y|y|^{-2}|y-\xi+x|^{-3+\varepsilon} \leqq z \varepsilon^{-1} r^{-2+\varepsilon}
$$


For $I_{2}$, one has

$$
I_{2} \leqq \int_{|y|<|y-\xi|} d^{3} y|x-y|^{-2}(1+|y|)^{-1-\varepsilon}(|y-\xi|+1)^{-2+2 \varepsilon} .
$$

Break $I_{2}$ into $I_{3}=\left\{y:|y-\xi|>\frac{1}{2} r\right\} \cap I_{2}$ and $I_{4}$, the remainder. For $I_{3}$,

$$
I_{3} \leqq z r^{-2+2 \varepsilon} \int d^{3} y|x-y|^{-2}(1+|y|)^{-1-\varepsilon} \leqq z \varepsilon^{-1} r^{-2+2 \varepsilon}(1+|x|)^{-\varepsilon} .
$$

For $I_{4}$, note that $|x-y|>\frac{1}{2} r$ and $|y| \leqq|x-y|$. Then,

$$
I_{4} \leqq z \int d^{3} y(r+|y|)^{-2}|y|^{-3+\varepsilon} \leqq z \varepsilon^{-1} r^{-2+\varepsilon} .
$$

Finally, since $1+|x|$ is either $\geqq$ or $\leqq$ than $1+|x-\xi|$, one obtains Assertion (2) from Eqs. (C.4.22-25) and also Assertion (3).

\section{D.1. The Stress-Energy Identity}

For a solution $c \in \mathbb{C}^{\delta}$ of Eq. (C.1.1), the a priori estimates of Sects. C.2-4 allow a calculation of the "forces" between the path components of the strong field region $U[c]\left(\kappa_{0}\left(\mathfrak{U}^{\delta}(c)\right)\right)$. The purpose here is to establish that these path components are in a region of fixed diameter.

Proposition D.1.1. Let $\delta_{1} \in\left(0, \frac{1}{4}\right)$ be as in Lemma C.4.1. Let $n \in \mathbb{Z}$. Let $\delta \in\left[0, \delta_{1}\right)$ and suppose that $c \in \mathbb{C}_{n}^{\delta}$ is a solution to Eq. (C.1.1) such that for some $\varepsilon>0$, $\mathfrak{A}^{\delta}(c) \geqq 4 \pi|n|+\varepsilon$. Let $\kappa_{0}(\cdot)$ be as in Lemma D.2.1. Then $\operatorname{diam} U[c]\left(\kappa_{0}\left(\mathfrak{H}^{\delta}(c)\right)\right)$ $\leqq \zeta\left(\mathfrak{A}^{\delta}(c)\right)\left(1+\varepsilon^{-2}\right)$, where $\zeta \in C^{0}([0, \infty),[0, \infty))$ is independent of $\varepsilon, c \in \mathfrak{C}^{\delta}$ and $\delta \in\left[0, \delta_{1}\right)$.

The remainder of this section, and Sects. D.2, 3 are occupied with the proof of Proposition D.1.1. To prove the proposition, it is sufficient to provide a bound on the distance between the path components of $U[c]\left(\kappa_{0}\right)$, since the diameter of any one component is bounded by $4 \mathfrak{U}(c) / \kappa_{0}^{-1}$ (Lemma C.2.1). This bound is derived in two steps. The first step, summarized in Lemma D.1.2, establishes that this maximum separation can be bounded by $\zeta\left(\mathfrak{U}^{\delta}(c)\right) \delta^{-2}$. A $\delta$-independent bound is derived in Sect. D.3 with information provided in Sect. D.2.

To begin, let $\kappa_{0}$ be as specified in Lemma C.2.1, and let $c=(A, \Phi) \in \mathbb{C}^{\delta}$ be a solution to Eq. (C.1.1). Let $U[c]$ denote $U[c]\left(\kappa_{0}\left(\mathfrak{U}^{\delta}(c)\right)\right)$ and let $\left\{U_{j}, j=1, \ldots, n[c]\left(\kappa_{0}\right)\right\}$ be its path components. Define for each $j$, the point $x_{j}$ by Eq. (C.2.1); $x_{j}$ is the center of $U_{j}$. Associate to $c$ the numbers

$$
d_{*}=\max \left\{\left|x_{j}\right|: j=1, \ldots, n[c]\left(\kappa_{0}\right)\right\},
$$

and

$$
\varrho=\varrho(c)=\max \left\{\left|x_{i}-x_{j}\right|: i, j=1, \ldots, n[c]\left(\kappa_{0}\right)\right\} .
$$

Lemma D.1.2. Let $\delta_{1}$ be given by Lemma C.4.1. Let $\delta \in\left(0, \delta_{1}\right)$ and let $c \in \mathfrak{C}^{\delta}$ be a solution to $E q$. (C.1.1). Define $d_{*}$ and $\varrho$ by Eq. (D.1.1). Then

$$
d_{*} \leqq \zeta\left(\mathfrak{U}^{\delta}(c)\right)(\varrho+1) \leqq \zeta^{\prime}\left(\mathfrak{U}^{\delta}(c)\right) \delta^{-2},
$$

where $\zeta, \zeta^{\prime} \in C^{0}([0, \infty),(0, \infty))$ are independent of $c \in \mathbb{C}^{\delta}$ and $\delta \in\left[0, \delta_{1}\right)$.

The proof of Lemma D.1.2 will be given shortly. The estimates for the proof are derived from the "stress-energy" identity in the next lemma: 
Lemma D.1.3. Let $\delta \in\left[0, \frac{1}{2}\right)$ and let $c=(A, \Phi) \in \mathbb{C}^{\delta}$ a solution to Eq. (C.1.1). Let $\left(f_{\alpha}, g_{\alpha}\right)_{\alpha=1}^{3}$ be the Ad $P$-valued components of $(f, g) \equiv\left(\sigma^{\delta} * F_{A}, D_{A} \Phi\right)$ with respect to the basis $\left\{d y^{\alpha}\right\}_{\alpha=1}^{3}$ of $T^{*}$. Then

$$
\frac{1}{2} \frac{\partial}{\partial y^{\alpha}}\left(|f|^{2}-|g|^{2}\right)-\frac{\partial}{\partial y^{\beta}}\left(\left(f_{\alpha}, f_{\beta}\right)-\left(g_{\alpha}, g_{\beta}\right)\right)+\delta \sigma^{-2} y^{\alpha}|f|^{2}=0 .
$$

Proof of Lemma D.1.3. Contract Eqs. (C.1.1a) and (C.1.2a) with $f$ and then add them, and then contract Eqs. (C.1.1b) and (C.1.2b) with $* g$ and subtract these. The result after some manipulation is Eq. (D.1.2).

Some corollaries of Lemma D.1.3, "virial theorems," are listed below.

Corollary D.1.4. Let $\delta \in\left[0, \frac{1}{2}\right)$ and let $c \in \mathfrak{C}^{\delta}$ be a solution to Eq. (C.1.1). Let $B \subset \mathbb{R}^{3}$ be an open ball of radius $R>0$ and center $x \in \mathbb{R}^{3}$. Let $n=\sum_{\alpha} n^{\alpha} d y^{\alpha}$ be the exterior normal to $\partial B$. Then

$$
\int_{B} d^{3} y\left\{|g|^{2}-|f|^{2}+2 \delta \sigma^{-2}\left(|y|^{2}-(y, x)\right)|f|^{2}\right\}=R \int_{\partial B} * n\left\{|g|^{2}-|f|^{2}+2\left|f_{n}\right|^{2}-2\left|g_{n}\right|^{2}\right\},
$$

where $(y, x)=\sum_{\alpha} y^{\alpha} x^{\alpha}$ and $f_{n}\left(g_{n}\right)=\sum_{\alpha} n^{\alpha} f_{\alpha}\left(\sum_{\alpha} n^{\alpha} g_{\alpha}\right)$.

Corollary D.1.5. Under the same assumptions as in Lemma D.1.3,

$$
\int_{\mathbb{R}^{3}} d^{3} y\left\{|g|^{2}-|f|^{2}+2 \delta \sigma^{-2}|y|^{2}|f|^{2}\right\}=0,
$$

and if $\delta>0$, then for each $\alpha=1,2,3$,

$$
\int_{\mathbb{R}^{3}} d^{3} y y^{\alpha} \sigma^{-2}|f|^{2}=0 \text {. }
$$

Proposition D.1.1 is proved by obtaining a $\delta$-independent estimate for $\varrho(c)$ from Eq. (D.1.4). An explicit calculation of the left-hand side of Eq. (D.1.4) in terms of the numbers $\left\{\mathfrak{a}_{j}, \mathfrak{n}_{j}\right\}$ of Lemmas C.3.1 and C.4.1 respectively is carried out in Sect. D.3. This calculation, summarized in Proposition D.3.2, gives the bound on $\varrho(c)$. The final task in this section is to prove Lemma D.1.2 and Corollaries D.1.4 and D.1.5.

Proof of Corollary D.1.4. To obtain Eq. (D.1.3), contract Eq. (D.1.2) with $y-x=\left(y^{\alpha}-x^{\alpha}\right)_{\alpha=1}^{3}$ and integrate the result over $B$. After an integration by parts (i.e. Stokes theorem), Eq. (D.1.3) is obtained.

Proof of Corollary D.1.5. For Eq. (D.1.4), take the limit in Eq. (D.1.3) with $B$ sequentially balls of increasing, integer radius, centered at $0 \in \mathbb{R}^{3}$. Lemmas C.2.1, C.3.1, and C.4.1 insure that the boundary term on the right-hand side of Eq. (D.1.4) tends uniformly to zero in the limit. For Eq. (D.1.5), integrate Eq. (D.1.2) over $\mathbb{R}^{3}$, and then integrate by parts.

Proof of Lemma D.1.2. Consider the left-hand inequality first. Suppose for the sake of argument that $d_{*}>16(\varrho+1)$. Let $x \in\left\{x_{j}\right\}$ be such that $|x|=d_{*}$. For some $R \in\left[\frac{1}{4} d_{*}, \frac{1}{8} d_{*}\right]$, let $B=\left\{y \in \mathbb{R}^{3}:|y-x| \leqq R\right\}$. Then $\operatorname{dist}\left(\left\{x_{j}\right\}, \mathbb{R}^{3} \backslash B\right)>\frac{1}{16} d_{*}$. Now 
contract Eq. (D.1.5) with $x=\left(x^{\alpha}\right)_{\alpha=1}^{3}$. The contribution from $B$ satisfies

since for $y \in B$,

$$
\int_{B} d^{3} y(x, y) \sigma^{-2}|f|^{2} \geqq \frac{1}{10} \int_{B} d^{3} y|f|^{2},
$$

$$
(x, y) \sigma^{-2}(y) \geqq \frac{1}{10} .
$$

Meanwhile, Lemmas C.2.1 and C.4.1 allow the contribution from $\mathbb{R}^{3} \backslash B$ to be estimated as follows:

$$
\begin{aligned}
\left.\left|\int_{\mathbb{R}^{3} \backslash B} d^{3} y(x, y) \sigma^{-2}\right| f\right|^{2} \mid & \leqq|x| \zeta\left(\mathfrak{U}^{\delta}(c)\right) \int_{|x-y|>R} d^{3} y|y|^{-1+2 \delta}(1+|y-x|)^{-4+1 / 16} \\
& \leqq \zeta\left(\mathfrak{H}^{\delta}(c)\right) d_{*}^{-1 / 2} .
\end{aligned}
$$

Further, for $y \in \partial B$, Lemmas C.2.1, C.3.1, and C.4.1 imply that

$$
\left(|f|^{2}+|g|^{2}\right)(y) \leqq \zeta\left(\mathfrak{H}^{\delta}(c)\right) d_{*}^{-4+2 \delta+1 / 16},
$$

and so Corollary D.1.4 asserts here that

$$
\int_{B} d^{3} y\left\{|g|^{2}-2|f|^{2}\right\} \leqq \zeta\left(\mathfrak{Q}^{\delta}(c)\right) d_{*}^{-1 / 2} .
$$

Above, the fact that $\delta_{1}<\frac{1}{4}$ has been used. Together with Eq. (D.1.5), Eqs. (D.1.6, 8, 10) imply that

$$
\int_{B} d^{3} y\left\{|g|^{2}+|f|^{2}\right\} \leqq \zeta\left(\mathfrak{I}^{\delta}(c)\right) d_{*}^{-1 / 2} .
$$

However, by construction, $B$ contains the centers, $x_{j}$, of each $U_{j}$, and so

$$
\kappa_{0}\left(\mathfrak{2}^{\delta}(c)\right) \leqq \int_{B} d^{3} y\left(|g|^{2}+|f|^{2}\right) .
$$

Thus, should it be true that $d_{*} \geqq 8(\varrho+1)$, then Eqs. (D.1.11, 12) imply that

$$
d_{*} \leqq \zeta\left(\mathfrak{Q}^{\delta}(c)\right) \text {. }
$$

This establishes the left-hand inequality in Lemma D.1.2.

To establish the right-hand inequality, a crucial fact is that there exists $R \in\left[\frac{1}{4} d_{*}, \frac{1}{2} d_{*}\right]$ such that the ball $B=\left\{y \in \mathbb{R}^{3}:|y-x| \leqq R\right\}$ has the following property: For all $j \in\left\{1, \ldots, n[c]\left(\kappa_{0}\right)\right\}$,

$$
\operatorname{dist}\left(x_{j} ; \partial B\right) \geqq\left(10 n[c]\left(\kappa_{0}\right)\right)^{-1} d_{*} .
$$

Indeed, the set of $R \in\left[\frac{1}{4} d_{*}, \frac{1}{2} d_{*}\right]$, where Eq. (D.1.14) fails for some $x_{j}$ has measure less than $\frac{1}{5} d_{*}$ (and it has at most $n[c]\left(\kappa_{0}\right)$ path components).

Contract both sides of Eq. (D.1.2) with $x$ and integrate the result over $B$. After an integration by parts, one obtains

$$
\frac{1}{2} \int_{\partial B}(x \cdot n)\left(|f|^{2}-|g|^{2}\right)-\int_{\partial B}\left(\left(f_{n}, f_{x}\right)-\left(g_{n}, g_{x}\right)\right)+\delta \int_{B} d^{3} y \sigma^{-2}(x, y)|f|^{2}=0 .
$$

Here $n=\left(n^{\alpha}\right)_{\alpha=1}^{3}$ is the exterior normal to $\partial B$ and for $v=x$ or $n, f_{v}\left(g_{v}\right)$ $\sum_{\alpha} v^{\alpha} f_{\alpha}\left(\sum_{\alpha} v^{\alpha} g_{\alpha}\right)$. Together, Eqs. (D.1.7, 9, and 15) imply the inequality

$$
\delta \int_{B} d^{3} y|f|^{2} \leqq \zeta\left(\mathfrak{H}^{\delta}(c)\right) d_{*}^{-3 / 4+2 \delta} \leqq \zeta\left(\mathfrak{H}^{\delta}(c)\right) d_{*}^{-1 / 2} .
$$


Here, the fact that $\delta<\delta_{1}<\frac{1}{4}$ has been used. Together, Eqs. (D.1.10,16) imply that $\delta \int_{B} d^{3} y\left(|f|^{2}+|g|^{2}\right) \leqq \zeta\left(\mathfrak{Q}^{\delta}(c)\right) d_{*}^{-1 / 2}$. But, by construction, $B$ contains at least one path component of $U[c]\left(\kappa_{0}\right)$, and so this last equation implies that $\delta \kappa_{0}\left(\mathfrak{U}^{\delta}(c)\right)$ $\leqq \zeta\left(\mathfrak{A}^{\delta}(c)\right) d_{*}^{-1 / 2}$, from which it follows that $d_{*} \leqq \zeta\left(\mathfrak{U}^{\delta}(c)\right) \delta^{-2}$, for $\zeta \in C^{0}([0, \infty)$, $[0, \infty))$, independent of $c \in \mathfrak{C}^{\delta}$ and $\delta \in\left(0, \delta_{1}\right)$. This last equation implies the righthand inequality in Lemma D.1.2 because of the triangle inequality's implication that

$$
2 \varrho \leqq d_{*}
$$

\section{D.2. The Cluster Decomposition}

To prove Proposition D.1.1, it is useful to group into clusters those points $\left\{x_{j}, j=1, \ldots, n[c]\left(\kappa_{0}\right)\right\}$ which are relatively close together. Two charges will be associated to each cluster. Physically, one should interpret one charge as determining the coupling of the cluster to the " $\Phi$ " field; and the other charge should be interpreted as determining the coupling of the cluster to the " $A$ " field.

Let $\delta_{1}$ be as in Lemma C.4.1 and let $\delta \in\left[0, \delta_{1}\right)$. Let $c=(A, \Phi) \in \mathfrak{C}^{\delta}$ be a solution to Eq. (C.1.1) and let $n=n[c]\left(\kappa_{0}\left(\mathfrak{A}^{\delta}(c)\right)\right)$ be defined for $\kappa_{0}$ as in Lemma C.2.1. Let $\Lambda=\Lambda(c)=\left\{x_{j}: j=1, \ldots, n\right\}$. For $\lambda \subset \Lambda$, denote by $m=m(\lambda)$ the number of elements of $\lambda$. Let $\varrho=\varrho(c)$ be the number from Eq. (D.1.1). Assume that $\varrho \geqq 2$.

For $r \in\left(0, \frac{1}{16}\right)$, define an $r$-cluster to be a non-empty subset $\lambda \subset \Lambda$ with the following properties:

(a) $\operatorname{diam} \lambda<\varrho^{1 / 2} r^{n-m+1}$,

(b) $\lambda$ is not strictly contained in any $\lambda^{\prime} \subset \Lambda$ which satisfies (a).

The number $r$ will be specified in Sect. D.3 as a function of $\mathfrak{U}^{\delta}(c)$.

For an $r$-cluster $\lambda$, define

$$
\varrho(\lambda)=\operatorname{dist}(\lambda, \Lambda \backslash \lambda) \text {. }
$$

The $r$-clusters in $\Lambda$ have the following properties:

Lemma D.2.1. Each $x \in \Lambda$ is contained in a unique r-cluster. Also, as $\varrho \geqq 2, \Lambda$ contains at least two r-clusters. If $\lambda$ is an r-cluster, then

$$
\varrho(\lambda) \geqq \frac{1}{2} \varrho^{1 / 2} r^{n-l} .
$$

Proof of Lemma D.2.1. Let $x \in \Lambda$. The first step is to prove that $x$ is in at least one $r$-cluster. To do this, define a group, $\mu \subset \Lambda$, to be a subset which satisfies Eq. (D.2.1a). Then $x$ is contained in the group $\mu=\{x\}$. Suppose that groups $\mu_{1}, \mu_{2}$ exist with $m=m\left(\mu_{1}\right) \geqq m\left(\mu_{2}\right)$ and $\mu_{2} \varsubsetneqq \mu_{1}$ and $x \in \mu_{1} \cap \mu_{2}$. Let $\mu=\mu_{1} \cup \mu_{2}$. If $z, w \in \mu$, then

$$
|z-w| \leqq|z-x|+|w-x|<2 \varrho^{1 / 2} r^{n-m+1}<\frac{1}{2} \varrho^{1 / 2} r^{n-l}<\varrho^{1 / 2} r^{n-m(\mu)+1} .
$$

Thus $\mu$ is a group. Therefore, the set of groups that contain $x$ is a partially ordered set (by inclusion), and so there is a maximal group which contains $x$. By definition, this maximal group is an $r$-cluster, and the construction shows that it is unique. Due to Eq. (D.2.1), every $r$-cluster $\lambda \subset \Lambda$ satisfies $\operatorname{diam}(\lambda) \leqq \frac{1}{4} \varrho^{1 / 2}$, and so $\Lambda$ must 
contain at least two, since $\operatorname{diam} \Lambda=\varrho$. Finally, if Eq. (D.2.3) were not true for $r$-clusters $\lambda$, $\mu$ with $m=m(\lambda) \geqq m(\mu)$, then $\tau=\lambda \cup \mu$ would be a group containing both $\lambda$ and $\mu$. Indeed, for any $x, y \in \tau$, one would have

$|x-y| \leqq \operatorname{diam} \lambda+\operatorname{diam} \mu+\operatorname{dist}(\lambda, \mu)$

$$
<2 \varrho^{1 / 2} r^{n-m+1}+\frac{1}{2} \varrho^{1 / 2} r^{n-m}<\varrho^{1 / 2} r^{n-m}<\varrho^{1 / 2} r^{n-m(\tau)+1} .
$$

To each cluster $\lambda \subset A$, associate the numbers

$$
\mathfrak{a}(\lambda)=\sum_{x_{j} \in \lambda} \mathfrak{a}_{j} \text { and } \mathfrak{n}(\lambda)=\sum_{x_{j} \in \lambda} \mathfrak{n}_{j}
$$

where $\mathfrak{a}_{j}$ and $\boldsymbol{n}_{j}$ are as specified in Lemmas C.3.1 and C.4.1, respectively. Also, associate to each cluster its center,

$$
X(\lambda)=m^{-1} \sum_{x \in \lambda} x
$$

and the open ball

$$
B(\lambda)=\left\{y \in \mathbb{R}^{3}:|y-X(\lambda)|<\varrho(\lambda) r^{1 / 3}\right\} .
$$

Lemma D.2.1 implies that for distinct $r$-clusters $\lambda, \mu$,

$$
\operatorname{diam}(\lambda)<2 \varrho(\lambda) r, \operatorname{dist}(\lambda, \partial B(\lambda)) \geqq \frac{1}{2} \varrho(\lambda) r^{1 / 3}, \operatorname{dist}(\mu, \partial B(\lambda)) \geqq \frac{1}{2} \varrho(\lambda) .
$$

An important step in the proof of Proposition D.1.1 is to identify the numbers $\mathfrak{a}(\lambda), \mathfrak{n}(\lambda)$ with integrals over $B(\lambda)$ of bilinear functionals of the curvatures of $c=(A, \Phi)$. Lemmas D.2.2 and D.2.3 accomplish this task.

Lemma D.2.2. Let $\delta_{1}$ be given by Lemma C.4.1. Let $\delta \in\left[0, \delta_{1}\right)$ and let $c \in \mathfrak{C}^{\delta}$ be a solution to Eq. (C.1.1). Let $\lambda$ be an r-cluster and define $(a(\lambda), n(\lambda))$ by Eq. (D.2.4). Then

(1) $\left|\mathfrak{a}(\lambda)-\frac{1}{8 \pi} \int_{B(\lambda)}\left\{|g|^{2}+|f|^{2}\right\}\right| \leqq \zeta\left(\mathfrak{A}^{\delta}(c)\right)\left(\delta+\varrho(\lambda)^{-1 / 2} r^{-1 / 3}\right)$,

(2) $|\mathfrak{n}(\lambda)| \leqq \mathfrak{a}(\lambda)+\zeta\left(\mathfrak{H}^{\delta}(c)\right)\left(\delta+\varrho(\lambda)^{-1 / 2} r^{-1 / 3}\right)$,

where $\zeta \in C^{0}([0, \infty),[0, \infty))$ is independent of $\lambda, c \in \mathbb{C}^{\delta}$ and $\delta \in\left[0, \delta_{1}\right)$.

Lemma D.2.3. Let $n \in \mathbb{Z}$. Let $\delta_{1}$ and $\delta$ be as in Lemma D.2.2, and let $c \in \mathfrak{C}_{n}^{\delta}$ be a solution to Eq. (C.1.1). Define $\left\{\mathfrak{a}_{j}\right\}$ and $\left\{\mathrm{n}_{j}\right\}$ by Lemmas C.3.1 and C.4.1, respectively.

$$
4 \pi \sum_{j} \mathfrak{a}_{j}=\mathfrak{Q}^{\delta}(c)-\delta \int d^{3} y \sigma^{-2}|y|^{2}|f|^{2}, \quad \text { and } \quad \sum_{j} \mathfrak{n}_{j}=n
$$

Proof of Lemma D.2.2. Write $c=(A, \Phi)$. To prove Assertion (1), contract Eq. (C.1.1b) with $\Phi$ and then integrate over $B(\lambda)$. Stokes theorem yields the identity

$$
\int_{B(\lambda)}|g|^{2}=\int_{\partial B(\lambda)} *(\Phi, g) \text {. }
$$


Let $R=\varrho(\lambda)^{1 / 2} r^{1 / 3}$. Observe that for $y \in \partial B(\lambda)$, Lemma C.3.1 and Eq. (D.2.7) imply that

$$
\left|(\Phi, g)-\sum_{j} \mathfrak{a}_{j}\right| y-\left.x_{j}\right|^{-2} d\left|y-x_{j}\right| \mid<\zeta\left(\mathfrak{A}^{\delta}(c)\right) R^{-3} .
$$

Together, Eqs. (D.2.8-9) assert that

$$
\left.\left|\int_{B(\lambda)}\right| g\right|^{2}-\sum_{j} a_{j} \int_{\partial B(\lambda)}\left|y-x_{j}\right|^{-2} * d\left|y-x_{j}\right| \mid<\zeta\left(\mathfrak{Q}^{\delta}(c)\right) R^{-1} .
$$

Then, Stokes theorem implies from Eq. (D.2.10) that

$$
\left.\left|\int_{B(\lambda)}\right| g\right|^{2}-4 \pi \mathfrak{a}(\lambda) \mid<\zeta\left(\mathfrak{H}^{\delta}(c)\right) R^{-1} .
$$

Next, Corollary D.1.4 and Lemmas C.2.1, C.3.1, and C.4.1 imply the bound

$$
\left|\int_{B|\lambda|}\left\{|g|^{2}-|f|^{2}\right\}\right|<\zeta\left(\mathfrak{A}^{\delta}(c)\right)\left\{\delta\left(1+(\varrho+R)^{1 / 16} R^{-1}\right)+\left(\varrho^{2 \delta}+R^{2 \delta}+1\right) R^{-1}\right\} .
$$

However, Lemma D.1.2 implies that

$$
(\varrho+R)^{1 / 16} R^{-1}+R^{2 \delta}+\varrho^{2 \delta}<1+\zeta\left(\mathfrak{H}^{\delta}(c)\right) \delta^{3 / 4},
$$

and since $\delta<\frac{1}{4}$, Eq. (D.2.12) actually asserts that

$$
\left|\int_{B(\lambda)}\left\{|g|^{2}-|f|^{2}\right\}\right| \leqq \zeta\left(\mathfrak{I}^{\delta}(c)\right)\left(\delta+R^{-1}\right) .
$$

Together, Eqs. (D.2.11) and (D.2.14) imply Assertion (1) of Lemma D.2.2.

To obtain Assertion (2) of Lemma D.2.2, first use the fact that for $y \in \partial B(\lambda)$, Lemmas C.4.1 and C.4.2 and Eq. (D.2.7) imply that

$$
\left|\left(\Phi, F_{A}\right)-\sum_{j} n_{j}\right| y-\left.x_{j}\right|^{-2} * d\left|y-x_{j}\right|-d \omega \mid \leqq \zeta\left(\mathfrak{H}^{\delta}(c)\right)\left(R^{-3}+\delta R^{-2}\right) .
$$

Thus, Stokes theorem and Eq. (D.2.15) imply that

$$
\left|\int_{\partial B(\lambda)}\left(\Phi, F_{A}\right)-4 \pi \mathfrak{n}(\lambda)\right| \leqq \zeta\left(\mathfrak{I}^{\delta}(c)\right)\left(\delta+R^{-1}\right) .
$$

Next, use Stokes theorem and Eq. (C.1.2a) to obtain the identity

$$
\int_{\partial B(\lambda)}\left(\Phi, F_{A}\right)=\int_{B(\lambda)}\left(g, * F_{A}\right) .
$$

But, by the triangle inequality,

$$
\left|\int_{B(\lambda)}\left(g, * F_{A}\right)\right| \leqq \frac{1}{2} \int_{B(\lambda)}\left\{|g|^{2}+|f|^{2}\right\},
$$

so Assertion (2) of Lemma D.2.2 is implied by Eqs. (D.2.16-18) and Assertion (1) of Lemma D.2.2.

Proof of Lemma D.2.3. For the first claim, note that Eq. (C.1.1b) and Lemma C.3.1 imply that

$$
4 \pi \sum_{j} \mathfrak{a}_{j}=\int_{\mathbb{R}^{3}}|g|^{2} .
$$


Now use Corollary D.1.5. For the second claim, note that Eqs. (A.1.4) and (C.1.2a) imply that

$$
4 \pi n=\int_{\mathbb{R}^{3}}\left(g, * F_{A}\right)=\lim _{R \rightarrow \infty} \int_{|y|=R}\left(\Phi, F_{A}\right) .
$$

This identity with Lemmas C.3.1, C.4.2, 3, 7 complete the proof.

Finally, one must identify the vector charges, $\left\{l_{j}\right\}$, of Lemma C.4.1. For an $r$-cluster $\lambda$, define $l(\lambda)=\sum_{j \in \lambda} l_{j}$. These are described in

Lemma D.2.4. Under the same assumptions as in Lemma D.2.2, let $\lambda$ be an r-cluster. Then

$$
|l(\lambda)| \leqq \zeta\left(\mathfrak{H}^{\delta}(c)\right)\left[\varrho(\lambda)^{-3 / 2}+\delta\right],
$$

where $\zeta \in C^{0}([0, \infty),[0, \infty))$ is independent of $\lambda, c \in \mathfrak{C}^{\delta}$, and $\delta \in\left[0, \delta_{1}\right)$.

Proof of Lemma D.2.4. As in Sect. C, let $\psi_{j}=\left(1+\left|x-x_{j}\right|\right)^{-5}$, and let $\pi_{j}=\psi_{j}\left(\sum_{k} \psi_{k}\right)^{-1}$. Define $q \in \Gamma\left(T^{*}\right)$ by Eq. (C.4.3). Note that $d * \sigma^{2 \delta} q=0$. Also,

$$
d \pi_{j}=5 \sum_{k: k \neq j} \pi_{j} \pi_{k}\left(\frac{d\left|x-x_{k}\right|}{\left(1+\left|x-x_{k}\right|\right)}-\frac{d\left|x-x_{j}\right|}{\left(1+\left|x-x_{j}\right|\right)}\right) .
$$

Write $l(\lambda)=\sum_{\alpha=1}^{3} l_{\alpha} d x^{\alpha}$. Then for any constant $\xi \in \mathbb{R}^{3}$,

$$
l^{\alpha}(\lambda)=* \int_{\mathbb{R}^{3}} d(y-\xi)^{\alpha} \wedge * \sum_{j \in \lambda} \pi_{j} q=-* \int_{\mathbb{R}^{3}}(y-\xi)^{a} d * \sum_{j \in \lambda} \pi_{j} q .
$$

The right-hand side of Eq. (D.2.20) has two terms,

$$
l(\lambda)=-\int_{\mathbb{R}^{3}}(y-\xi)^{\alpha} \sum_{j \in \lambda} d \pi_{j} \wedge * q+2 \delta \int_{\mathbb{R}^{3}}(y-\xi)^{\alpha} \sigma^{-1} d \sigma \wedge * \sum_{j \in \lambda} \pi_{j} q .
$$

Finally, observe that Eq. (D.2.19) implies that

$$
\sum_{j \in \lambda} d \pi_{j}=\sum_{j \in \lambda} \sum_{k \notin \lambda} \pi_{j} \pi_{k}\left(\frac{d\left|x-x_{k}\right|}{\left(1+\left|x-x_{k}\right|\right)}-\frac{d\left|x-x_{j}\right|}{\left(1+\left|x-x_{j}\right|\right)}\right) .
$$

Evaluate Eq. (D.2.21) by considering the two possibilities, $|X(\lambda)|<\varrho(\lambda) r^{1 / 3}$ and the converse. For the first case, take $\xi=0$. For the second case, take $\xi=X(\lambda)$. In either case, Eq. (C.4.11) must be used with Eq. (D.2.22) to get

$$
\begin{aligned}
\left|\sum_{j \in \lambda} d \pi_{j} \wedge * q\right| \leqq & \zeta\left(\mathfrak{U}^{\delta}(c)\right) \sum_{j \in \lambda} \sum_{k \notin \lambda}\left(1+\left|x-x_{j}\right|\right)^{-5 / 2}\left(1+\left|x-x_{k}\right|\right)^{-5 / 2} \\
& \cdot\left(\left(1+\left|x-x_{j}\right|\right)^{-1}+\left(1+\left|x-x_{k}\right|\right)^{-1}\right) .
\end{aligned}
$$

The remaining details are straightforward and left to the reader.

\section{D.3. The Interaction of Clusters}

To obtain from Eq. (D.1.4) a $\delta$-independent bound for the number $\varrho(c)$, it is necessary to consider associations of the points in $\Lambda$ on length scales which are 
$O(\varrho(c))$ as well as the cluster length scales which are $O\left(\varrho^{1 / 2}(c)\right)$. By mimicking the definition of an $r$-cluster, define an $r$-molecule to be a subset, $\Theta \subset \Lambda$, with the following properties:

(a) $\operatorname{diam} \Theta<2 \varrho r^{n-m+1}$, where $m=m(\Theta)=$ the number of elements in $\Theta$.

(b) $\Theta$ is not strictly contained in any $\Theta^{\prime} \subset \Lambda$ which also satisfies (a).

For an $r$-molecule, define

$$
\varrho(\Theta)=\operatorname{dist}(\Theta, \Lambda \backslash \Theta) .
$$

In analogy with Lemma D.2.1, one has

Lemma D.3.1. Each $r$-cluster, $\mu \subset \Lambda$ is contained in a unique r-molecule. As $\varrho>0$, there exists at least two r-molecules. If $\Theta$ is an $r$-molecule, then $\varrho(\Theta) \geqq \varrho r^{n-m(\Theta)}$.

Proof of Lemma D.3.1. Mimic the proof of Lemma D.2.1.

The crucial estimate in the proof of Proposition D.1.1 involves the following a priori estimate.

Proposition D.3.2. Let $\delta_{1}$ be as in Lemma C.4.1. Let $\delta \in\left[0, \delta_{1}\right)$ and let $c \in \mathbb{C}^{\delta}$ be a solution to $E q$. (C.1.1). Let $\varrho=\varrho(c)$ be as in Eq. (D.1.1). Let $r \in\left(0, \frac{1}{2}\right)$; and if $\lambda \subset \Lambda(c)$ is an r-cluster, define $(\mathfrak{a}(\lambda), \mathfrak{n}(\lambda))$ by Eq. (D.2.4) and $X(\lambda)$ by Eq. (D.2.5). Then

$$
\begin{aligned}
\int_{\mathbb{R}^{3}} d^{3} y & \left\{|g|^{2}-|f|^{2}+2 \delta \sigma^{-2}|f|^{2}\right\} \geqq-\varrho^{-3 / 2} r^{-2 n} \zeta\left(\mathfrak{U}^{\delta}(c)\right) \\
& +4 \pi\left[1-r^{1 / 3} \zeta\left(\mathfrak{A}^{\delta}(c)\right)\right] \Sigma^{\prime} \frac{(\mathfrak{a}(\mu) \mathfrak{a}(\lambda)-\mathfrak{n}(\mu) \mathfrak{n}(\lambda))}{|X(\mu)-X(\lambda)|} \\
& +\delta \kappa_{0}\left(\mathfrak{U}^{\delta}(c)\right)\left(1-\zeta\left(\mathfrak{U}^{\delta}(c)\right) \varrho^{-1 / 2} r^{-2 n}\right) .
\end{aligned}
$$

Here, $\zeta(\cdot) \in C^{0}([0, \infty),[0, \infty))$ is independent of $c \in \mathbb{C}^{\delta}$ and $\delta \in[0,1)$; and $\sum^{\prime}$ means to sum over all ordered pairs, $(\mu, \lambda)$, of r-clusters for which $\mu$ and $\lambda$ are in distinct $r$-molecules.

This result will be proved shortly.

Proof of Proposition D.1.1, given Proposition D.3.2. Corollary D.1.5 asserts that the right-hand side of Eq. (D.3.3) is zero. Choose $r=\left[4+4 \zeta\left(\mathfrak{U}^{\delta}(c)\right)\right]^{-3}$. Then Eq. (D.3.3) asserts that

$$
\begin{aligned}
0 \geqq & -\varrho^{-3 / 2} \zeta\left(\mathfrak{U}^{\delta}(c)\right)+2 \pi \Sigma^{\prime} \frac{(\mathfrak{a}(\mu) \mathfrak{a}(\lambda)-\mathfrak{n}(\mu) \mathfrak{n}(\lambda))}{|X(\mu)-X(\lambda)|} \\
& +\delta \kappa_{0}\left(\mathfrak{H}^{\delta}(c)\right)\left(1-\zeta\left(\mathfrak{A}^{\delta}(c)\right) \varrho^{-1 / 2}\right) .
\end{aligned}
$$

According to Lemma D.2.3,

$$
4 \pi \sum_{j} \mathfrak{a}_{j} \geqq(1-\delta) \mathfrak{A}^{\delta}(c) \geqq(1-\delta) 4 \pi|n|+\frac{1}{2} \varepsilon,
$$

in fact,

$$
4 \pi \sum_{j} \mathfrak{a}_{j} \geqq 4 \pi\left|\sum_{j} \mathfrak{n}_{j}\right|+\frac{1}{2} \varepsilon-\mathfrak{U}^{\delta}(c) \delta .
$$


Since each $x_{j} \in \Lambda$ is contained in a unique $r$-cluster, Eq. (D.3.5) asserts that

$$
4 \pi \sum_{r-\text { clusters }} \mathfrak{a}(\lambda) \geqq 4 \pi\left|\sum_{r \text { clusters }} \mathrm{n}(\lambda)\right|+\frac{1}{2} \varepsilon-\mathfrak{Q}^{\delta}(c) \delta .
$$

The second assertion of Lemma D.2.2 and Eq. (D.3.6) now allow only two possibilities. The first possibility is that there exists an $r$-cluster $\lambda$ for which

$$
\mathfrak{a}(\lambda) \geqq \frac{1}{2} n[c]\left(\kappa_{0}\right)^{-1} \varepsilon+|\mathfrak{n}(\lambda)|-\zeta\left(\mathfrak{H}^{\delta}(c)\right)\left(\delta+\varrho^{-1 / 2}\right) .
$$

If Eq. (D.3.7) is not satisfied, then Eq. (D.3.6) and Lemma D.2.2 imply that the $\mathrm{n}(\lambda)$ 's are not all of the same sign. Because Lemma D.2.1 implies that

$$
\mathfrak{a}(\lambda) \geqq \frac{1}{8 \pi} \kappa_{0}\left(\mathfrak{H}^{\delta}(c)\right)-\zeta\left(\mathfrak{H}^{\delta}(c)\right)\left(\delta+\varrho^{-1 / 2}\right),
$$

either of the two cases imply with Eq. (D.3.4) and Lemma D.2.2 that

$$
\begin{aligned}
0 \geqq & -\varrho^{-3 / 2} \zeta\left(\mathfrak{H}^{\delta}(c)\right)+\pi \varrho^{-1}\left(n(c)\left(\kappa_{0}\right)+4 \pi\right)^{-1} \kappa_{0} \min \left(\varepsilon, \kappa_{0}\right) \\
& +\delta \kappa_{0}\left(\mathfrak{H}^{\delta}(c)\right)\left(1-\zeta\left(\mathfrak{H}^{\delta}(c)\right) \varrho^{-1 / 2}\right) .
\end{aligned}
$$

Equation (D.3.9) provides a $\delta$-independent bound for $\varrho(c)$ : $\varrho(c)$ $\leqq \zeta\left(\mathfrak{U}^{\delta}(c)\right) \max \left(1, \varepsilon^{-2}\right)$, where $\zeta \in C^{0}([0, \infty],[0, \infty))$ is independent of $c \in \mathfrak{C}^{\delta}$ and $\delta \in\left[0, \delta_{1}\right)$, as required.

The remainder of this section contains the proof of Proposition D.3.2. For this purpose, some additional terminology is required. First, for an $r$-molecule $\Theta$, define its center, $x(\Theta) \subset \mathbb{R}^{3}$ as follows: First, let $w=\left\{y \in \mathbb{R}^{3}: \operatorname{dist}(y, \Theta)<\varrho(\Theta) r\right\}$, and then let

$$
x(\Theta)=\left(\int_{w} \sigma^{-2}|f|^{2}\right)^{-1}\left(\int_{w} \sigma^{-2} y|f|^{2}\right) .
$$

Let

$$
B(\Theta)=\left\{y \in \mathbb{R}^{3}:|y-x(\Theta)|<\varrho(\Theta) r^{1 / 3}\right\} .
$$

Notice that because

$$
\operatorname{diam} \Theta \leqq 2 \varrho(\Theta) r
$$

one has

$$
\operatorname{dist}(\Theta, \partial B(\Theta)) \geqq \frac{1}{3} \varrho(\Theta) r^{1 / 3}, \quad \text { and } \quad \operatorname{dist}(\Lambda \backslash \Theta, \partial B(\Theta)) \geqq \frac{1}{2} \varrho(\Theta) .
$$

To each $r$-molecule, $\Theta$, associate the number

$$
s(\Theta)=\int_{B(\Theta)}\left\{|g|^{2}-|f|^{2}+2 \delta \sigma^{-2}|y|^{2}|f|^{2}\right\} .
$$

Let $T=\mathbb{R}^{3} \backslash \bigcup_{\Theta} B(\Theta)$ and define

$$
s(T)=\int_{T}\left\{|g|^{2}-|f|^{2}+2 \delta \sigma^{-2}|y|^{2}|f|^{2}\right\} .
$$


Each $s(\Theta)$, above, is evaluated using Lemma D.1.3. For this task, define for each $j=1, \ldots, n[c]\left(\kappa_{0}\right)$

$$
\phi_{j}(y)=-\mathfrak{a}_{j}\left|y-x_{j}\right|^{-1} \text { and } \quad \eta_{j}(y)=-\mathfrak{n}_{j}\left|y-x_{j}\right|^{-1} .
$$

Lemmas C.2.1 and C.3.1 assert that for $y \in T$,

$$
|g-\Phi(\Phi, g)|(y) \leqq \zeta\left(\mathfrak{A}^{\delta}(c)\right) \sum_{j}\left|y-x_{j}\right|^{-3}
$$

and

$$
\left|(\Phi, g)-\sum_{j} d \phi_{j}\right|(y)<\zeta\left(\mathfrak{H}^{\delta}(c)\right) \sum_{j}\left|y-x_{j}\right|^{-3} .
$$

Also, for each $j=1, \ldots, n[c]\left(\kappa_{0}\right)$, the 1 -form $\omega_{j}$ is defined by Eq. (C.4.13). Lemmas C.2.1, C.3.1, and C.4.3 assert that

$$
|f-\Phi(\Phi, f)|(y) \leqq \zeta\left(\mathfrak{r}^{\delta}(c)\right) \sum_{j}\left|y-x_{j}\right|^{-3}
$$

and

$$
\left|(\Phi, f)-\sigma^{-\delta} \sum_{j} d \eta_{j}-\sigma^{\delta} \sum_{j} * d \omega_{j}\right|(y) \leqq \zeta\left(\mathfrak{P}^{\delta}(c)\right) \sum_{j}\left|y-x_{j}\right|^{-3} .
$$

For notational convenience, define the real 1-forms

$$
q=\sum_{j} d \phi_{j} \text { and } \quad p=\sum_{j} d \eta_{j} .
$$

The first step in the proof of Proposition (D.3.6) is

Lemma D.3.3. Under the same assumption as in Proposition D.3.2, let $\Theta \subset \Lambda(c)$ be an r-molecule. Then

$$
\begin{aligned}
s(\Theta) \geqq & \varrho(\Theta) r^{1 / 3} \int_{\partial B(\Theta)} * n\left(|q|^{2}-\sigma^{2 \delta}|p|^{2}+2\left(\sigma^{2 \delta} p_{n}^{2}-q_{n}^{2}\right)\right) \\
& +\int_{B(\Theta)} d^{3} y(y, x(\Theta)) \sigma^{-2}|f|^{2}-\zeta\left(\mathfrak{U}^{\delta}(c)\right)\left[\varrho^{-3 / 2} r^{-2 n}+\delta \varrho^{-1 / 2} r^{-2 n}\right],
\end{aligned}
$$

where $\zeta \in C^{0}([0, \infty),[0, \infty))$ is independent of $c \in \mathfrak{C}^{\delta}$ and $\delta \in\left[0, \delta_{1}\right)$.

Proof of Lemma D.3.3. Use Corollary D.1.4 with $x=x(\Theta)$ and $B=B(\Theta)$. That corollary with Eqs. (D.3.13, D.3.17, 18) yield the stated inequality with the addition to the right-hand side of the term:

$$
\varrho(\Theta) r^{1 / 3} \int_{\partial B(\Theta)} * n\left(-2(p \cdot v)-|v|^{2}+2 p_{n} v_{n}+2\left|v_{n}\right|^{2}\right),
$$

where $\nu=\sigma^{\delta} \sum_{j} * d \omega_{j}$.

To evaluate Eq. (D.3.20), consider that for $x \in T$ and $x_{j} \in \lambda$, an $r$-cluster, one has

$$
|d| x-\left.x_{j}\right|^{-1}-d|x-X(\lambda)|^{-1}\left|\leqq z \cdot \varrho^{-1 / 2} r^{-n}\right| x-\left.X(\lambda)\right|^{-2},
$$


where $z$ is independent of $x, x_{j}$, and $\lambda$. Thus for $x \in T, v$ is estimated via Lemma C.4.1:

$$
\begin{aligned}
\left|v+\sum_{\lambda} * l(\lambda) \wedge d\right| x-\left.X(\lambda)\right|^{-1} \mid \leqq & \zeta\left(\mathfrak{P}^{\delta}(c)\right)\left[\varrho^{-1 / 2} r^{-n} \sum_{\lambda}|x-X(\lambda)|^{-2}\right. \\
& \left.+\delta(1+|x|)^{1 / 32} \sum_{\lambda}|x-X(\lambda)|^{2-1 / 16}\right]
\end{aligned}
$$

where $\zeta(\cdot) \in C^{0}([0, \infty),[0, \infty))$ is independent of $c \in \mathbb{C}^{\delta}$ and $\delta \in\left[0, \delta_{1}\right)$. Using Eq. (D.3.21) with Lemmas D.1.2 and D.2.4, the extra term, Eq. (D.3.20), is readily seen to bound $-\zeta\left(\mathfrak{H}^{\delta}(c)\right) \cdot\left[\varrho^{-3 / 2} r^{-2 n}+\delta \varrho^{-1 / 2} r^{-2 n}\right]$ as required.

In order to further analyze $s(\Theta)$, it is convenient to introduce for each $r$-molecule, $\Theta$, real 1-forms

$$
q(\Theta)=\sum_{j: x_{j} \in \Theta} d \phi_{j}, \quad p(\Theta)=\sum_{j: x_{j} \in \Theta} d \eta_{j}
$$

Be aware that as long as $y \notin \Theta$,

$$
d q(\Theta)=0, \quad d * q(\Theta)=0,
$$

and

$$
d p(\Theta)=0, \quad d * p(\Theta)=0 .
$$

The second step in the proof of Proposition D.3.2 is to isolate the contribution to each $s(\Theta)$ from the $r$-molecule $\Theta$. For this one needs Lemmas D.3.4-5 below:

Lemma D.3.4. Under the same assumptions as in Proposition D.3.2, let $\Theta, \Pi$ be distinct $r$-molecules. Let $v(\cdot)$ denote $q(\cdot)$ or $p(\cdot)$ of Eq. (D.3.22) and let $\left\{\mathfrak{b}_{j}\right\}$ denote $\left\{\mathfrak{a}_{j}\right\}$ or $\left\{\mathfrak{n}_{j}\right\}$ respectively. Then

$$
\begin{gathered}
\varrho(\Theta) r^{1 / 3} \int_{\partial B(\Theta)} * n\left(|v(\Pi)|^{2}-2 v_{n}(\Pi)^{2}\right)=\int_{B(\Theta)} d^{3} y|v(\Pi)|^{2} . \\
\varrho(\Theta) r^{1 / 3} \int_{\partial B(\Theta)} * n\left((v(\Pi), v(\Theta))-2 v_{n}(\Pi) v_{n}(\Theta)\right) \\
=\int_{B(\Theta)} d^{3} y(v(\Pi), v(\Theta))-4 \pi \sum^{\prime} \frac{\mathfrak{b}_{j} \mathfrak{b}_{k}}{\left|x_{j}-x_{k}\right|^{3}}\left(x_{j}-x(\Theta), x_{j}-x_{k}\right),
\end{gathered}
$$

where $\sum^{\prime}$ means to sum over all $x_{j} \in \Theta$ and $x_{k} \in \Pi$.

Proof of Lemma D.3.4. Equation (D.3.23a, b) implies that for $r$-molecules $\Theta, \Pi$ and for $y \notin \Theta \cup \Pi$

$$
\frac{\partial}{\partial y^{\alpha}}(v(\Theta), v(\Pi))-\frac{\partial}{\partial y^{\beta}}\left(v_{\alpha}(\Theta) v_{\beta}(\Pi)+v_{\beta}(\Theta) v_{\alpha}(\Pi)\right)=0 .
$$

The derivation of Eq. (D.3.24) is formally the same as the derivation of Eq. (D.1.2). For Assertion (1), take $\Theta=\Pi$ above and then contract Eq. (D.3.24) with $y-x(\Theta)$. Then integrate the result over $B(\Theta)$. An integration by parts completes the job. For Assertion (2), contract Eq. (D.3.24) with $y-x(\Theta)$ and then 
for each $\varepsilon>0$, integrate over $B(\Theta) \backslash\left\{y \in \mathbb{R}^{3}: \operatorname{dist}(y, \Theta)<\varepsilon\right\}$. Integrate by parts and observe that the $\varepsilon \rightarrow 0$ limit is achieved uniformly; and results in Assertion (2).

To use Lemma D.3.4 to estimate $s(\Theta)$ requires the elimination of the factors of $\sigma^{2 \delta}$ :

Lemma D.3.5. Under the same assumptions of Proposition D.3.2, let $\Theta$ be an r-molecule. Define p by Eq. (D.3.19). Then

$$
\varrho(\Theta) r^{1 / 3} \int_{\partial B(\Theta)}\left|1-\sigma^{2 \delta}\right||p|^{2} \leqq \zeta\left(\mathfrak{U}^{\delta}(c)\right)\left(\delta \varrho^{-1 / 2} r^{-2 n}+\varrho^{-3 / 2} r^{-2 n}\right),
$$

and

$$
\int_{T}\left|1-\sigma^{2 \delta}\right||p|^{2} \leqq \zeta\left(\mathfrak{I}^{\delta}(c)\right)\left(\delta \varrho^{-1 / 2} r^{-2 n}+\varrho^{-3 / 2} r^{-2 n}\right) .
$$

Here $\zeta(\cdot) \in C^{0}([0, \infty),[0, \infty))$ is independent of $c \in \mathbb{C}^{\delta}$ and $\delta \in\left[0, \delta_{1}\right)$.

Proof of Lemma D.3.5. Use Eq. (D.3.16) along with Lemma D.1.12; this last implying that if $y \in \mathbb{R}^{3}$ has $|y| \leqq 4 d_{*}^{2}$, then

$$
\left|1-\sigma^{2 \delta}(y)\right| \leqq \zeta\left(\mathfrak{U}^{\delta}(c)\right) \delta^{7 / 8} .
$$

The third step in the proof of Proposition D.3.2 is the evaluation of $s(T)$. This evaluation requires

Lemma D.3.6. Under the same assumptions as in Proposition D.3.2,

$$
s(T)>\int_{T} d^{3} y\left(|q|^{2}-|p|^{2}+2 \delta \sigma^{-2}|y|^{2}|p|^{2}\right)-\zeta\left(\mathfrak{A}^{\delta}(c)\right)\left[\varrho^{-3 / 2} r^{-2 n}+\delta \varrho^{-1 / 2} r^{-2 n}\right],
$$

where $\zeta(\cdot) \in C^{0}([0, \infty),[0, \infty))$ is independent of $c \in \mathbb{C}^{\delta}$ and $\delta \in\left[0, \delta_{1}\right)$.

Proof of Lemma D.3.6. This is an immediate consequence of Eqs. (D.3.17, 18, 21).

The fourth step in the proof is to isolate the contribution of $s(T)$ from each $r$-molecule $\Theta$ :

Lemma D.3.7. Under the same assumptions as in Proposition D.3.2, let $\Theta$ be an $r$-molecule. Define $p(\Theta), q(\Theta)$ as in Eq. (D.3.22). Then

$$
\begin{aligned}
& \int_{\mathbb{R}^{3} \backslash \boldsymbol{B ( \Theta )}} d^{3} y\left(|q(\Theta)|^{2}-|p(\Theta)|^{2}\right) \\
& \quad=-\varrho(\Theta) r^{1 / 3} \int_{\partial B(\Theta)} * n\left(|q(\Theta)|^{2}-|p(\Theta)|^{2}+2\left(p_{n}(\Theta)^{2}-q_{n}(\Theta)^{2}\right)\right) .
\end{aligned}
$$

Proof of Lemma D.3.7. Use Eq. (D.3.24) with $\Theta=\Pi$. Contract with $y-x(\Theta)$ and then integrate over $\mathbb{R}^{3} \backslash B(\Theta)$. Do this with $v=q$, then $v=p$ and subtract the resulting two equations. Then integrate by parts.

The fifth and crucial step in the proof of Proposition D.3.2 is to compute the interaction energy between $r$-molecules. The result is

Lemma D.3.8. Under the same assumptions as in Proposition D.3.2, let $\Theta, \Pi$ be distinct r-molecules. Then

$$
\int_{\mathbb{R}^{3}}(q(\Theta), q(\Pi))=4 \pi \sum^{\prime} \frac{\mathfrak{a}_{j} \mathfrak{a}_{k}}{\left|x_{j}-x_{k}\right|},
$$


and

$$
\int_{\mathbb{R}^{3}}(p(\Theta), p(\Pi))=4 \pi \sum^{\prime} \frac{\mathrm{n}_{j} \mathfrak{n}_{k}}{\left|x_{j}-x_{k}\right|},
$$

where $\Sigma^{\prime}$ is the sum over all $x_{j} \in \Theta$ and $x_{k} \in \Pi$.

Proof of Lemma D.3.8. Use the defining equations, Eqs. (D.3.16, 22) and the fact that $4 \pi|x-y|^{-1}$ is the Green's function for $d^{*} d$.

Together Lemmas D.3.3-8 imply the following inequality:

$$
\begin{aligned}
& \int_{\mathbb{R}^{3}} d^{3} y\left\{|g|^{2}-|f|^{2}+2 \delta \sigma^{-2}|y|^{2}|f|^{2}\right\} \geqq 4 \pi \sum^{\prime} \frac{\left(\mathfrak{a}_{j} \mathfrak{a}_{k}-\mathfrak{n}_{j} \mathfrak{n}_{k}\right)}{\left|x_{j}-x_{k}\right|} \\
& \quad+4 \pi \sum_{\Theta} \sum_{\Theta}^{\prime} \frac{\left(\mathfrak{a}_{j} \mathfrak{a}_{k}-\mathfrak{n}_{j} \mathfrak{n}_{k}\right)}{\left|x_{j}-x_{k}\right|^{3}}\left(x_{j}-x(\Theta), x_{j}-x_{k}\right) \\
& \quad+\delta \sum_{\Theta}\left\{\int_{B(\Theta)} d^{3} y \sigma^{-2}(y, x(\theta))|f|^{2}\right\}-\zeta\left(\mathfrak{U}^{\delta}(c)\right)\left(\delta \varrho^{-1 / 2} r^{-2 n}+\varrho^{-3 / 2} r^{-2 n}\right) .
\end{aligned}
$$

Here $\Sigma^{\prime}$ means to sum over all $x_{j}, x_{k}$ in distinct $r$-molecules. $\sum_{\Theta}$ means to sum over all $r$-molecules, and $\sum_{\Theta}^{\prime}$ means to sum over all $x_{j} \in \Theta$ and $x_{k} \in \Lambda \backslash \Theta$.

Additional progress requires

Lemma D.3.9. Under the assumptions of Proposition D.3.2, let $\Theta$ be an r-molecule. Then

(1) $\int_{B(\Theta)} d^{3} y \sigma^{-2}(y, x(\Theta))|f|^{2} \geqq-\zeta\left(\mathfrak{U}^{\delta}(c)\right)\left(\varrho^{-1 / 2} r^{-2 n}+\delta \varrho^{-1 / 4} r^{-2 n}\right)$. If $|x(\Theta)|>\varrho(\Theta) r^{1 / 3}$, then

(2) $\int_{B(\Theta)} d^{3} y \sigma^{-2}(y, x(\Theta))|f|^{2} \geqq \frac{1}{8} \kappa_{0}\left(\mathfrak{U}^{\delta}(c)\right)-\zeta\left(\mathfrak{U}^{\delta}(c)\right)\left(\varrho^{-1 / 2} r^{-2 n}+\delta \varrho^{-1 / 4} r^{-2 n}\right)$.

Here $\zeta(\cdot) \in C^{0}([0, \infty),[0, \infty))$ is independent of $c \in \mathbb{C}^{\delta}$ and $\delta \in\left[0, \delta_{1}\right)$. The function $\kappa_{0}$ is defined in Lemma C.2.1.

Proof of Lemma D.3.9. To prove Assertion (1), note that Lemmas C.4.1 and D.1.2 with Eqs. (D.3, 10, 13, 25) imply that

$$
\int_{B(\Theta)} d^{3} y \sigma^{-2}(y, x(\Theta))|f|^{2} \geqq|x(\Theta)|^{2} \int_{B(\Theta)} d^{3} y \sigma^{-2}|f|^{2}-\zeta\left(\mathfrak{U}^{\delta}(c)\right)\left(\varrho^{-1 / 2} r^{-2 n}+\delta \varrho^{-1 / 4} r^{-2 n}\right) \text {. }
$$

To prove Assertion (2), observe that if $|x(\Theta)| \geqq \varrho(\Theta) r^{1 / 3}$, then

$$
\int_{B(\Theta)} \sigma^{-2}|f|^{2} \geqq \frac{1}{2} \sigma^{-2}(x(\Theta)) \int_{B(\Theta)} d^{3} y|f|^{2} .
$$

In addition, Corollary D.1.4 asserts with Eqs. (D.3.17, 18, 21) and Lemmas D.2.2 and D.2.4 that

$$
\begin{gathered}
\frac{1}{2} \int_{B(\Theta)} d^{3} y|f|^{2} \geqq \frac{1}{4} \int_{B(\Theta)} d^{3} y\left(|f|^{2}+|g|^{2}\right)-\delta \int d^{3} y \sigma^{-2}(y, x(\Theta))|f|^{2} \\
-\zeta\left(\mathfrak{U}^{\delta}(c)\right) \varrho^{-1 / 2} r^{-2 n}
\end{gathered}
$$


Therefore, because $|x(\Theta)| \geqq 1$,

$$
\int_{B(\Theta)} d^{3} y \sigma^{-2}(y, x(\Theta))|f|^{2} \geqq \frac{1}{8} \int_{B(\Theta)} d^{3} y\left(|f|^{2}+|g|^{2}\right)-\zeta\left(\mathfrak{U}^{\delta}(c)\right) \varrho^{-1 / 2} r^{-2 n} .
$$

As $B(\Theta)$ contains at least one $x_{j} \in \Lambda$, this last equation implies Assertion (2).

Proof of Proposition D.3.2(completion). Due to Eq. (D.3.13), there exists at least one $r$-molecule, $\Theta$, with $|x(\Theta)|>\varrho(\Theta) r^{1 / 3}$. that

Equations (D.3.26) and (D.3.27) along with Lemma D.3.9 force the conclusion

$$
\begin{aligned}
& \int_{\mathbb{R}^{3}} d^{3} y\left\{|g|^{2}-|f|^{2}+2 \delta \sigma^{-2}|y|^{2}|f|^{2}\right\} \\
& >4 \pi \sum^{\prime} \frac{\left(\mathfrak{a}_{j} \mathfrak{a}_{k}-\mathfrak{n}_{j} \mathfrak{n}_{k}\right)}{\left|x_{j}-x_{k}\right|}+4 \pi \sum_{\Theta} \sum_{\Theta}^{\prime} \frac{\left(\mathfrak{a}_{j} \mathfrak{a}_{k}-\mathfrak{n}_{j} \mathfrak{n}_{k}\right)}{\left|x_{j}-x_{k}\right|^{3}}\left(x_{j}-x(\Theta), x_{j}-x_{k}\right) \\
& \quad+\delta \kappa_{0}\left(\mathfrak{H}^{\delta}(c)\right)-\zeta\left(\mathfrak{U}^{\delta}(c)\right)\left(\delta \varrho^{-1 / 2} r^{-2 n}+\varrho^{-3 / 2} r^{-2 n}\right) .
\end{aligned}
$$

In order to obtain finally Proposition D.3.2, it is necessary to rearrange the sums in Eq. (D.3.28) into sums over $r$-clusters. Let $\lambda$ be an $r$-cluster and let $x_{j} \in \lambda$. Then

$$
\left|x_{j}-X(\lambda)\right|<\varrho^{1 / 2} r \text {. }
$$

If $\mu$ is an $r$-cluster in a distinct $r$-molecule from $\lambda$, and if $x_{k} \in \mu$, then

$$
|| x_{j}-x_{k}|-| X(\lambda)-X(\mu) \|<2 \varrho^{1 / 2} r .
$$

By rewriting the sums in Eq. (D.3.28) as double sums, first over $r$-clusters, and second over points in $r$-clusters, one obtains with Eq. (D.3.30) that

$$
\sum^{\prime} \frac{\left(\mathfrak{a}_{j} \mathfrak{a}_{k}-\mathfrak{n}_{j} \mathfrak{n}_{k}\right)}{\left|x_{j}-x_{k}\right|} \geqq \sum^{\prime \prime} \frac{\mathfrak{a}(\lambda) \mathfrak{a}(\mu)-\mathfrak{n}(\lambda) \mathfrak{n}(\mu)}{|X(\lambda)-X(\mu)|}-\zeta\left(\mathfrak{U}^{\delta}(c)\right) \varrho^{-3 / 2} r^{-2 n} .
$$

Here, $\Sigma^{\prime \prime}$ is the sum over pairs $(\lambda, \mu)$ of $r$-clusters in distinct $r$-molecules. In addition,

$$
\begin{aligned}
\sum_{\Theta} \sum_{\Theta}^{\prime} & \frac{\left(\mathfrak{a}_{j} \mathfrak{a}_{k}-\mathfrak{n}_{j} \mathfrak{n}_{k}\right)}{\left|x_{j}-x_{k}\right|^{3}}\left(x_{j}-x(\Theta), x_{j}-x_{k}\right) \geqq-r^{1 / 3} \sum^{\prime \prime} \frac{(\mathfrak{a}(\lambda) \mathfrak{a}(\mu)-\mathfrak{n}(\lambda) \mathfrak{n}(\mu))}{|X(\lambda)-X(\mu)|} . \\
& -\zeta\left(\mathfrak{Q}^{\delta}(c)\right)\left(\varrho^{-3 / 2} r^{-2 n}+\delta \varrho^{-1 / 2} r^{-2 n}\right) .
\end{aligned}
$$

Here, Assertion (2) of Lemma D.2.2 has been used. Proposition D.3.2 is a direct consequence of Eqs. (D.3.28, 31-32).

\section{D.4. The Convergence of Min-Max for $\mathfrak{Z}$}

The a priori estimate that is provided by Proposition D.1.1 is the key result for the proof of Proposition C.1.1. This section consists of the proof of said proposition.

Proof of Proposition C.1.1. It is assumed that $\mathfrak{U}_{\mathfrak{F}}>4 \pi|n|+\varepsilon$ for some $\varepsilon>0$. According to Lemma B.1.2, each $\mathfrak{U}_{\mathfrak{F}}^{\delta} \geqq \mathfrak{U}_{\mathfrak{F}}>4 \pi|n|+\varepsilon$ too. Now Proposition D.1.1 asserts that if $\delta \in\left(0, \delta_{1}\right)$, then the number $\varrho\left(c^{\delta}(\mathfrak{F})\right)$ of Eq. (D.1.1) is bounded independently of $\delta \in\left[0, \delta_{1}\right)$. Lemma D.1.2 provides a $\delta$-independent bound for the number $d_{*}\left(c^{\delta}(\mathfrak{J})\right)$ of Eq. (D.1.1). Now, Lemmas C.2.1, C. 3.1, and C.4.1 provide a 
constant $z=z\left(\mathfrak{A}_{\mathfrak{F}}-4 \pi|n|\right)<\infty$ with the property that if $\delta \in\left[0, \delta_{1}\right)$, then $c^{\delta}(\mathfrak{F})=(A, \Phi)$ has curvatures which satisfy

$$
\left(\left|F_{A}\right|+\left|D_{A} \Phi\right|\right)(x) \leqq z(1+|x|)^{-2+1 / 16}
$$

Equation (D.4.1) implies that the numbers $\left\{\left\|\nabla \mathfrak{U}_{c}\right\|_{*}: c=c^{\delta}(\mathfrak{F})\right.$ and $\left.\delta \in\left(0, \delta_{1}\right)\right\}$ tend uniformly to zero as $\delta \rightarrow 0$. This fact allows one to invoke Lemma B.3.2. Said lemma provides a subsequence $\left\{c_{i}=c^{\delta}(\mathfrak{F}): \delta<i^{-1}\right\} \subset\left\{c^{\delta}(\mathfrak{F})\right\}$ and a sequence $\left\{g_{i}\right\}$ C(5 such that $\left(A_{i}, \Phi_{i}\right)=g_{i} c_{i}$ converges strongly in $L_{1 ; \text { loc }}^{2}\left(T^{*} \times \mathfrak{s u}(2)\right) \times L_{2 ; \text { loc }}^{2}(\operatorname{Ad} P)$ to $(A, \Phi) \in L_{1 ; 1 \mathrm{loc}}^{2}\left(T^{*} \times \mathfrak{s u}(2)\right) \times L_{2 ; \text { loc }}^{2}(\operatorname{Ad} P)$ with $(A, \Phi)$ in $\mathbb{C}$ being a critical point of A. However, Eq. (D.4.1) and Lemma A.4.6 of [9] imply that the sequence of curvatures, $\left\{\left(F_{A_{i}}, D_{A_{i}} \Phi_{i}\right)\right\}$, converges strongly in $L^{2}$ to $\left(F_{A}, D_{A} \Phi\right)$. This means that $\left\{g_{i} c_{i}\right\}$ converges to $c$ in the $L_{1 ; \text { loc }}^{2}$ topology on $\mathcal{C}$. The $L_{1 ; \text { loc }}^{2}$ convergence with Lemma B.1.2 guarantees that $\mathfrak{Q}(c)=\mathfrak{U}_{\mathfrak{F}}$.

The convergence of $\left\{g_{i} c_{i}\right\}$ to $c$ can be assumed to be with respect to the $C^{\infty}$ topology on $\mathfrak{C}$. The argument goes as follows: As the set $\left\{\mathfrak{U}\left(c_{i}\right)\right\}$ is bounded, $\mathbb{R}^{3}$ can be covered by a uniform set of open balls, $\left\{B_{\alpha}\right\}$, of fixed radius such that in each $B_{\alpha}$, and for each $i$, there exists $h_{\alpha i} \in C^{\infty}\left(B_{\alpha} ; \mathrm{SU}(2)\right)$ which puts $A_{i}$ into the Hodge gauge in $B_{\alpha}$. That is, if $h_{\alpha i}\left(A_{i}, \Phi_{i}\right)$ is written $\left(A_{\alpha i}, \Phi_{\alpha i}\right)$, then

$$
d^{*} A_{\alpha i}=0 \text { in } B_{\alpha} \text {, and }\left(n_{\alpha}, A_{\alpha i}\right)=0 \text { on } \partial B_{\alpha},
$$

where $n_{\alpha}$ is the unit normal to $B_{\alpha}$. Further,

$$
\left\|A_{\alpha i}\right\|_{2,1 ; B_{\alpha}} \leqq z\left\|F_{A_{i}}\right\|_{2 ; B_{\alpha}},
$$

where $z$ is independent of $i$ and $\alpha$. This fact is a direct consequence of $\mathrm{K}$. Uhlenbeck's compactness theorems in [19]. Her theorems also provide for each $\alpha$, an $h_{\alpha} \in C^{\infty}\left(B_{\alpha} ; \mathrm{SU}(2)\right)$ such that in $B_{\alpha}, h_{\alpha}(A, \Phi) \equiv\left(A_{\alpha}, \Phi_{\alpha}\right)$ also satisfies Eqs. (D.4.2,3). Because $\left\{\left(A_{i}, \Phi_{i}\right)\right\}$ converges strongly in $L_{1 ; \text { loc }}^{2}$ to $(A, \Phi)$, the implicit function theorem in [19] allows that no generality is lost in assuming that in each $B_{\alpha},\left\{\left(A_{\alpha i}, \Phi_{\alpha i}\right)\right\}$ converges strongly in $L_{1}^{2}$ to $\left(A_{\alpha}, \Phi_{\alpha}\right)$. The advantage of the Hodge gauge of Eq. (D.4.2) is that in each $B_{\alpha}$, and for each $i$, Eq. (C.1.1) is a set of elliptic equations for the pair $\left(A_{\alpha i}, \Phi_{\alpha i}\right)$. The equations are $i$-dependent, but bootstrap theorems in [26, cf. Chap. 6] still apply, and one can conclude that in each $B_{\alpha}$, $\left(A_{\alpha i}, \Phi_{\alpha i}\right)$ converges to $\left(A_{\alpha}, \Phi_{\alpha}\right)$ in $C^{\infty}$. As a consequence, the set of transition functions $\left\{g_{\alpha \beta i}=h_{\alpha i} h_{\beta i}^{-1}\right\} \subset C^{\infty}\left(B_{\alpha} \cap B_{\beta}: S U(2)\right)$ converges in $C^{\infty}$ to $g_{\alpha \beta}=h_{\alpha} h_{\beta}^{-1}$. Now the construction in [19] as generalized in the appendix of [22] produces for each $i$, a $C^{\infty}$ bundle isomorphism $\left\{\eta_{\alpha i}: B_{\alpha} \rightarrow \mathrm{SU}(2)\right\}$ such that: (1) For each $i$ and pair $(\alpha, \beta)$, $\eta_{\alpha i} g_{\alpha \beta i} \eta_{\beta i}^{-1}=g_{\alpha \beta}$ in $U_{\alpha} \cap U_{\beta}$; and (2) for each $\alpha$, the sequence $\left\{\eta_{\alpha i}\right\} \subset C^{\infty}\left(B_{\alpha}\right)$ converges. Now, for each $i$ define $g_{i}^{\prime} \in \mathfrak{G}$ by setting $g_{i}^{\prime}(y)=\left(h_{\alpha}^{-1} \eta_{\alpha i} h_{\alpha i} g_{i}\right)(y)$ when $y \in B_{\alpha}$. The preceding discussion can be summarized by the statements that each $g_{i}^{\prime}$ is well defined, and that $\left\{g_{i}^{\prime} c_{i}\right\}$ converges strongly to $c$ in $\mathbb{C}$.

Now, let $\Pi: \mathbb{C} \rightarrow \mathfrak{B}$ denote the projection. Then $\left\{\Pi\left(g_{i}^{\prime} c_{i}\right)\right\}$ converges strongly to $\Pi(c)$ in $\mathfrak{B}$. For each $i$, there exists $q_{i} \in \mathrm{SU}(2) \subset\left(\mathfrak{5}\right.$ such that $q_{i} \Pi\left(g_{i}^{\prime} c_{i}\right)=c_{i}$. As $\mathrm{SU}(2)$ is compact, $\left\{q_{i}\right\}$ has a subsequence, $\left\{q_{j}\right\}$, which converges in $\mathrm{SU}(2)$ to $q \in \mathrm{SU}(2)$. Then the corresponding subsequence, $\left\{c_{j}\right\}$, converges in $\hat{\mathfrak{B}}$ to $q^{-1} \Pi(c) \equiv c(\mathfrak{F})$. This completes the proof of Proposition C.1.1. 


\section{E.1. The Neighborhood of the Moduli Space}

For $n \in \mathbb{Z}$, the infimum of $\mathfrak{A}$ on $\hat{\mathfrak{B}}_{n}$ is $4 \pi|n|$, and $\mathfrak{A}(c)=4 \pi|n|$ if and only if $c \in \mathfrak{M}_{n}=\left\{(A, \Phi) \in \hat{\mathfrak{B}}_{n}: F_{A}=\operatorname{sign}(n) * D_{A} \Phi\right\}$. The purpose of this section is to prove the following proposition:

Proposition E.1.1. For each $n \in \mathbb{Z}$, there exists $\varepsilon>0$ and a homotopy $m:[0,1]$ $\times \widehat{\mathfrak{B}}_{n} \rightarrow \hat{\mathfrak{B}}_{n}$ with the properties: (1) For all $t \in[0,1], \mathfrak{m}(t, \cdot)$ restricted to $\hat{\mathfrak{M}}_{n}$ is the identity; and (2) $\mathfrak{m}(1, \cdot): \widehat{\mathfrak{B}}_{n} \cap \mathfrak{A}^{-1}([4 \pi|n|, 4 \pi|n|+\varepsilon)) \rightarrow \mathfrak{M}_{n}$.

Corollary E.1.2. Let $n \in \mathbb{Z}$. There exists $\varepsilon>0$ such that if $\mathfrak{F}$ is a homotopy invariant family of compact subsets of $\mathfrak{B}_{n}$ with $\mathfrak{H}_{\mathfrak{F}}<4 \pi|n|+\varepsilon$, then $\mathfrak{A}_{\mathfrak{F}}=4 \pi|n|$ and there exists $F \in \mathfrak{F}$ with $F \subset \mathfrak{M}_{n}$.

The proof of Proposition E.1.1 follows closely the author's original existence proof for $\mathfrak{M}_{n}$ as it appears in [11]. An analog of Proposition E.1.1 for the YangMills functional on $S^{4}$ is proved in [22]. The proof here is modelled closely after the proof in [22]. For the proof, restrict attention to the cases $n \geqq 0$, as the $n<0$ cases are obtainable from the former by reversing the orientation of $\mathbb{R}^{3}$.

For $n \in \mathbb{Z}$, let $c \in \hat{\mathfrak{B}}_{n}$. Because (see $[12,13]$ )

$$
\mathfrak{A}(c)=\frac{1}{2}\left\|* F_{A}-D_{A} \Phi\right\|_{2}^{2}+4 \pi|n|,
$$

it is the $L^{2}$-norm of

$$
G(c)=\left(* F_{A}-D_{A} \Phi, 0\right) \in L^{2}(Q),
$$

which measures how close a given $c$ is to $\hat{\mathfrak{M}}_{n}$. For $c \in \mathfrak{C}$, define $G(c) \in L^{2}(Q)$ by Eq. (E.1.1) also.

Proposition E.1.1 is proved in two steps. The first step is summarized by

Lemma E.1.3. Given $0 \leqq n \in \mathbb{Z}$, there exists $\varepsilon>0$, a continuous homotopy $\mathrm{m}_{1}:[0,1]$ $\times \widehat{\mathfrak{B}}_{n} \rightarrow \hat{\mathfrak{B}}_{n}$ and a continuous map $R: \hat{\mathfrak{B}}_{n} \rightarrow[0, \infty)$ such that

(1) If $c \in \hat{\mathfrak{M}}_{n}$, then $\mathrm{m}_{1}(t, c)=c$ for all $t \in[0,1]$.

(2) If $c \in \mathfrak{B}_{n}$ and $\mathfrak{A}(c)<4 \pi n+\varepsilon$, then $G\left(\mathfrak{m}_{1}(1, c)\right)$ has compact support in $\left\{x \in \mathbb{R}^{3}:|x|<R(c)\right\}$ and $\left\|G\left(\mathrm{~m}_{1}(1, c)\right)\right\|_{2} \leqq\|G(c)\|_{2}$.

For the second step, introduce for each $n \in \mathbb{Z}$ the space $\mathfrak{E}_{n}=\left\{c \in \mathfrak{B}_{n}\right.$ : $\left.\|G\|_{6 / 5}<\infty\right\}$. Topologize $\mathfrak{E}_{n}$ by the inclusion $\mathfrak{E}_{n} \subset \mathfrak{B}_{n} \times[0, \infty)$ which sends $c$ to $\left(c,\|G(c)\|_{6 / 5}\right)$. Define $\hat{\mathfrak{E}}_{n}=\mathfrak{E}_{n} \cap \hat{\mathfrak{B}}_{n}$.

Lemma E.1.4. Let $0 \leqq n \in \mathbb{Z}$. There exists $\varepsilon>0$ and a homotopy $\mathrm{m}_{2}:[0,1] \times \widehat{\mathfrak{E}}_{n} \rightarrow \hat{\mathfrak{E}}_{n}$ such that

(1) If $c \in \hat{\mathfrak{M}}_{n}$, then $\mathrm{m}_{2}(t, c)=c$ for all $t \in[0,1]$.

(2) If $c \in \widehat{\mathfrak{E}}_{n}$ and $\mathfrak{A}(c)<4 \pi n+\varepsilon$, then as a function of $t \in[0,1], G\left(\mathrm{~m}_{2}(t, c)\right)$ $=(1-t) G(c)$.

Proof of Proposition E.1.1., assuming Lemmas E.1.3. and E.1.4. Let $\varepsilon$ be as in Lemma E.1.3. Notice that $\mathrm{m}_{1}(1, \cdot)$ maps $\left\{c \in \widehat{B}_{n}: \mathfrak{A}(c)<4 \pi n+\varepsilon\right\}$ continuously into the set $\left\{c \in \hat{\mathfrak{E}}_{n}: \mathfrak{A}(c)<4 \pi n+\varepsilon\right\}$. Let $\delta$ denote $\frac{1}{2}$ times the minimum of the $\varepsilon$ 's in Lemmas E.1.3 and E.1.4. Define $m$ as follows: Let $\alpha(c)=\beta\left(\delta^{-1}(\mathfrak{A}(c)-4 \pi n)\right)$, where 
$\beta \in C_{0}^{\infty}([0, \infty) ;[0,1])$ is identically 1 if $t \leqq \frac{1}{2}$ and identically 0 if $t \geqq \frac{3}{4}$. Let $(t, c)$ $\in[0,1] \times \hat{\mathfrak{B}}_{n}$ and set

$$
m(t, c)=\left\{\begin{array}{lll}
m_{1}(2 t, c) & \text { if } t \in\left[0, \frac{1}{2}\right] \\
m_{2}\left(\alpha(c)(2 t-1), m_{1}(1, c)\right) & \text { if } t \in\left[\frac{1}{2}, 1\right] \quad \text { and if } \alpha(c)>0 \\
m_{1}(1, c) & \text { if } t \in\left[\frac{1}{2}, 1\right] \text { and if } \alpha(c)=0
\end{array}\right.
$$

The proofs of Lemmas E.1.3 and E.1.4 occupy the rest of this section. To begin these proofs, it is necessary to introduce certain extra facts from [8]. To each $c \in \mathbb{C}$, associate the first order differential operators $\mathfrak{D}_{c}, \mathfrak{D}_{c}^{*}: \Gamma_{0}(Q) \rightarrow \Gamma_{0}(Q)$ which send $\psi=(a, \phi) \in \Gamma_{0}(Q)$ to

$$
\begin{aligned}
& \mathfrak{D}_{c} \psi=\left(* D_{A} a+[\Phi, a]-D_{A} \phi, D_{A} * a+[\Phi, \phi]\right), \\
& D_{c}^{*} \psi=\left(* D_{A} a-[\Phi, a]-D_{A} \phi, * D_{A} * a-[\Phi, \phi]\right) .
\end{aligned}
$$

These operators are formal $L^{2}$ adjoints of each other. Both are of the type that was discussed in Sect. C.2 of [9]. Associate to each $c \in \mathbb{C}$, the Hilbert space $H_{c}(Q)$ as defined in Sect. B.6 of [9]; the completion of $\Gamma_{0}(Q)$ in the norm, $\|\cdot\|_{c}$, which is defined by the inner product $\langle\psi, \eta\rangle_{c}=\left\langle\nabla_{A} \psi, \nabla_{A} \eta\right\rangle_{2}+\langle[\Phi, \psi],[\Phi, \eta]\rangle_{2}$. Then according to Lemma C.2.1 of [9], both $\mathfrak{D}_{c}$ and $\mathfrak{D}_{c}^{*}$ extend to bounded, Fredholm operators from $H_{c}(Q)$ to $L^{2}(Q)$. In fact, more is true: The sets $H(Q)$ $=\left\{H_{c}(Q): c \in \mathfrak{B}\right\}$ and $L(Q)=\left\{(c, \psi) \in \mathfrak{B} \times L^{2}(Q)\right\}$ are given $C^{0}$-vector bundle structures by Proposition B.6.3 of [9]. Lemma C.2.2 of [9] asserts that the assignment of $c \in \mathfrak{B}$ to $\mathfrak{D}_{c}$ and $\mathfrak{D}_{c}^{*}$ define continuous sections, $\mathfrak{D}$ and $\mathfrak{D}^{*}$, over $\mathfrak{B}$ of $\operatorname{Hom}(H(Q), L(Q))$. Let $\overline{\mathfrak{D}}_{c}$ and $\overline{\mathfrak{D}}_{c}^{*}$ be respectively the fibre-wise adjoints of $\mathfrak{D}_{c}$ and $\mathfrak{D}_{c}^{*}$. They define continuous sections, $\overline{\mathfrak{D}}$ and $\overline{\mathfrak{D}}^{*}$, over $\mathfrak{B}$ of $\operatorname{Hom}(L(Q), H(Q)$ ).

Given $\varepsilon>0$, define for each $0 \leqq n \in \mathbb{Z}$ the space $\mathfrak{B}_{n, \varepsilon}=\left\{b \in \mathfrak{B}_{n}: \mathfrak{A}(b)<4 \pi n+\varepsilon\right\}$. Similarly, define $\widehat{\mathfrak{B}}_{n, \varepsilon}$.

Lemma E.1.5. For each $0 \leqq n \in \mathbb{Z}$, there exist constants $\varepsilon, \mu>0$ and a function $v \in C^{0}\left(\mathfrak{B}_{n, \varepsilon} ;(0,1)\right)$ such that whenever $c \in \mathfrak{B}_{n, \varepsilon}$,

$$
\left\|\mathfrak{D}_{c}^{*}\right\| \geqq \mu \quad \text { and } \quad\left\|\overline{\mathfrak{D}}_{c}\right\| \geqq v(c) .
$$

Proof of Lemma E.1.5. According to Proposition 9.1 of [8], the vector space ker $\overline{\mathfrak{D}}_{c}$ is isomorphic to ker $\mathfrak{D}_{c}^{*}$. Proposition C.2.3 of [9] asserts that $\varepsilon>0$ exists such that when $c \in \mathfrak{B}_{n, \varepsilon}$, then $\operatorname{ker} \mathfrak{D}_{c}^{*}=\emptyset$. The existence of the constant $\mu$ follows from Lemma C.2.10 with the Wietzenbock formula for $\mathfrak{D}_{c}^{*}$, Eq. (6.13) of [8]. The existence of $v(c)>0$ such that $\left\|\bar{D}_{c}\right\|>v(c)$ follow from the fact that Range $\left(\overline{\mathfrak{D}}_{c}\right)$ is closed in $H_{c}(Q)$ (cf. Theorem IV.5.2 of [27]). The continuity of the assignment of $c \in \mathfrak{B}$ to $\overline{\mathfrak{D}}_{c}$ implies that $v(\cdot)$ can be taken to be continuous on $\mathfrak{B}_{n, \varepsilon}$.

For each $c \in \mathfrak{B}$, introduce the space $\Gamma^{c}=H_{c}(Q) \cap \Gamma(Q)$ with the induced topology. This $\Gamma^{c}$ is a Fréchet space.

Lemma E.1.6. Fix $c \in \mathfrak{B}$. Then the assignment of $\psi \in \Gamma^{c}$ to $c+\psi$ defines a continuous map from $\Gamma^{c}$ into $\mathfrak{C}$. The assignment of $(\psi, \eta) \in \Gamma^{c} \times \Gamma^{c}$ to $\mathfrak{D}_{c+\psi}^{*} \eta$ or $\mathfrak{D}_{c+\psi} \eta$ $\in L^{2}(Q) \cap \Gamma^{c}$ defines a smooth map; the assignment of $(\psi, \eta) \in \Gamma^{c} \times\left(L^{2}(Q) \cap \Gamma(Q)\right)$ to $\mathfrak{D}_{c+\psi} \eta \in H_{c}(Q)^{*} \cap \Gamma(Q)$ and to $\overline{\mathfrak{D}}_{c+\psi} \eta \in \Gamma^{c}$ define smooth maps. 
Proof Lemma E.1.6. The first assertion follows from Proposition A.4.3. The second assertion follows using Lemma B.6.4 of [9]. The third assertion claims that the map which sends $(\psi, \eta) \in \Gamma^{c} \times\left(L^{2}(Q) \cap \Gamma(Q)\right)$ to the linear functional $\left\langle\eta, \mathfrak{D}_{c+\psi}^{*}(\cdot)\right\rangle_{2}: H_{c}(Q) \rightarrow \mathbb{R}$ is $C^{\infty}$. This fact follows from Lemma B.6.4 of [9] which implies that the assignment of $(\sigma, \tau) \in \underset{2}{\times} \mathrm{H}_{c}(\operatorname{Ad} P)$ to $\langle[\sigma, \tau], \cdot\rangle_{2}: \Gamma_{0}(\operatorname{Ad} P) \rightarrow \mathbb{R}$ extends to define a smooth map of $\times_{2} H_{c}(\operatorname{Ad} P)$ into $H_{c}(\operatorname{Ad} P)^{*}$. For the final claim, define a map from $\Gamma^{c} \times \Gamma^{c} \times\left(L^{2}(Q) \cap \Gamma(Q)\right)$ to $H_{c}(Q)^{*} \cap \Gamma(Q)$ by assigning $(\psi, u, \eta)$ to the linear functional $\langle u, \cdot\rangle_{c+\psi}-\left\langle\eta, \mathfrak{D}_{c+\psi}(\cdot)\right\rangle_{2}$. This gives a smooth map into $H_{c}(Q)^{*}$, and the implicit function theorem implies the final claim.

For $c \in \mathbb{C}$ and $\psi=(a, \phi) \in \Gamma^{c}$, one has $G(c+\psi)=G(c)+\operatorname{Im} \mathfrak{D}_{c} \psi+\frac{1}{2}\{\psi, \psi\}$, where

$$
\{\psi, \psi\}=(*[a, a]-2[a, \phi], 0) \in \Gamma(Q),
$$

and

$$
\operatorname{Im}(a, \phi)=(a, 0) \in \Gamma(Q) .
$$

Lemma B.6.4 of [9] implies that the assignment of $\psi \in \Gamma^{c}$ to $\{\psi, \psi\}$ and to $\langle\{\psi, \psi\}, \cdot\rangle_{2}$ defines smooth maps from $\Gamma^{c}$ into $L^{2}(Q) \cap \Gamma(Q)$ and $H_{c}(Q)^{*} \cap \Gamma(Q)$, respectively. In particular, this means that the assignment of $\psi \in \Gamma^{c}$ to $G(c+\psi)$ $\in L^{2}(Q) \cap \Gamma(Q)$ gives a smooth map.

Let $\varepsilon$ be as in Lemma E.1.5 and let $c \in \mathfrak{B}_{n, \varepsilon}$. Define

$$
\bar{\Gamma}^{c}=\left\{\psi \in \Gamma^{c}: \mathfrak{U}(c+\psi)<4 \pi n+\varepsilon\right\} .
$$

Due to Lemma E.1.6, $\bar{\Gamma}^{c}$ is open in $\Gamma^{c}$.

Lemma E.1.7. Let $\varepsilon$ be as in Lemma E.1.5 and let $v \in C^{0}\left(\mathfrak{B}_{n, \varepsilon} ;(0,1)\right)$ be as defined there also. Let $\Pi: \mathfrak{C} \rightarrow \mathfrak{B}$ be the projection. There exists a continuous map $\delta: \mathfrak{B}_{n, \varepsilon / 2}$ $\rightarrow(0, \infty)$ with the following significance: For each $c \in \mathfrak{B}_{n, \varepsilon / 2}$ let $\mathfrak{D}_{c}^{\prime}=\left\{u \in L^{2}(Q)\right.$ $\left.\cap \Gamma(Q):\|u\|_{2}<\delta(c)\right\}$. Then for each $c \in \mathfrak{B}_{n, \varepsilon / 2}$, there exists an open neighborhood $\mathfrak{D}_{c}$ of 0 in $\bar{\Gamma}^{c}$ with a unique, smooth map $\tau_{c}: \mathfrak{D}_{c} \times \mathfrak{D}_{c}^{\prime} \rightarrow L^{2}(Q) \cap \Gamma(Q)$ which sends $(0,0)$ to 0 and which satisfies at each $(\psi, u) \in \mathfrak{D}_{c} \times \mathfrak{D}_{c}^{\prime}$,

(1) $G\left(c+\psi+\overline{\mathfrak{D}}_{c+\psi} \tau_{c}(\psi, u)\right)=G(c+\psi)+u$.

(2) $\left\|\tau_{c}(\psi, u)\right\|_{2} \leqq z v(\Pi(c+\psi))^{-2}\|u\|_{2}$, where $z<\infty$ is independent of $c, \psi$, and $u$.

Proof of Lemma E.1.7. For each $\psi \in \bar{\Gamma}^{c}$, consider the map $T(\psi, \cdot)$ which sends $\eta \in L^{2}(Q)$ to $T(\psi, \eta)=\mathfrak{D}_{c+\psi} \overline{\mathfrak{D}}_{c+\psi} \eta+\frac{1}{2}\left\{\overline{\mathfrak{D}}_{c+\psi} \eta, \overline{\mathfrak{D}}_{c+\psi} \eta\right\}$, as a map from $L^{2}(Q)$ to $L^{2}(Q)$. Note that $T(\psi, \cdot)$ is $C^{\infty}, T(\psi, 0)=0$, and that the differential of $T(\psi, \cdot)$ at $0 \in L^{2}(Q)$ sends $\eta \in L^{2}(Q)$ to $\mathfrak{D}_{c+\psi} \overline{\mathfrak{D}}_{c+\psi} \eta$. According to Lemma E.1.5, this differential is surjective and so the implicit function theorem provides the unique, smooth inverse, $T(\psi, \cdot)^{-1}$ to $T(\psi, \cdot)$ as a map from a neighborhood of $0 \in L^{2}(Q)$ into $L^{2}(Q)$. Lemma B.6.4 of [9] implies that

$$
\left\|T(\psi, \eta)-\mathfrak{D}_{c+\psi} \overline{\mathfrak{D}}_{c+\psi} \eta\right\|_{2} \leqq z\|\eta\|_{2}^{2},
$$

with $z$ independent of $c \in \mathfrak{B}_{n, \varepsilon / 2}$ and $\psi \in \bar{\Gamma}^{c}$. As $v(\cdot)$ of Lemma E.1.5 is continuous on $\mathfrak{B}_{\varepsilon}$, it follows from Eq. (E.1.5) that $T(\psi, \cdot)^{-1}$ exists as a smooth map from $\mathfrak{Q}_{c, \psi} \equiv\left\{u \in L^{2}(Q):\|u\|_{2}<\delta(\Pi(c+\psi))\right\}$ into $L^{2}(Q)$, where $\delta: \mathfrak{B}_{n, \varepsilon / 2} \rightarrow(0, \infty)$ is continuous. By replacing $\delta$ by $\frac{1}{2} \delta$, one obtains readily that for admissible $(c, \psi)$ and 
$u \in \mathfrak{Q}_{c, \psi}$,

$$
\left\|T^{-1}(\psi, u)\right\|_{2} \leqq z v(\Pi(c+\psi))^{-2}\|u\|_{2},
$$

and that if $T(\psi, \eta)=u$ and $\eta$ satisfies Eq. (E.1.6) replacing $T^{-1}(\psi, u)$, then $\eta=T^{-1}(\psi, u)$. Furthermore, the fact that the assignment of $(\psi, \eta) \in \bar{\Gamma}^{c}$ $\times\left(L^{2}(Q) \cap \Gamma(Q)\right)$ to $T(\psi, \eta) \in L^{2}(Q) \cap \Gamma(Q)$ defines a $C^{\infty}$ map with uniform estimates implies via Hamilton's inverse function theorem [28] that for each $c \in \mathfrak{B}_{n, \varepsilon / 2}$, there exists a neighborhood $\mathfrak{D}_{c} \subset \bar{\Gamma}^{c}$ of $0 \in \bar{\Gamma}^{c}$ such that $T^{-1}(\psi, \cdot)$ is a smooth map on $\mathfrak{Q}_{c}$ for all $\psi \in \mathfrak{D}_{c}$. Let $\mathfrak{D}_{c}^{\prime}=\mathfrak{Q}_{c} \cap \Gamma(Q)$. Then this implicit function theorem implies additionally that the assignment of $(\psi, u) \in \mathfrak{D}_{c} \times \mathfrak{D}_{c}^{\prime}$ to $\tau_{c}(\psi, u)$ $\equiv T^{-1}(\psi, u)$ defines, as required, a smooth map into $L^{2}(Q) \cap \Gamma(Q)$.

The application of Lemma E.1.7 is for a specific $u \in L^{2}(Q) \cap \Gamma(Q)$. For this, one requires

Lemma E.1.8. Let $\varepsilon$ be as in Lemma E.1.5 and let $\delta \in C^{0}\left(\mathfrak{B}_{n, \varepsilon} ;(0,1)\right)$ be given. Then there exists $\varrho \in C^{0}\left(\mathfrak{B}_{n, \varepsilon} ;(0,1)\right)$ such that for all $c \in \mathfrak{B}_{n, \varepsilon}$ and all $r>\varrho(c)$,

$$
\left\|\left(1-\beta_{r}\right) G(c)\right\|_{2}<\delta(c),
$$

where $\beta_{r} \in C_{0}^{\infty}\left(\mathbb{R}^{3}\right)$ is as defined in Sect. A.3.

Proof of Lemma E.1.8. If Eq. (E.1.7) holds at $c \in \mathfrak{B}$, for some $\varrho<\infty$, then it holds in an open neighborhood, $\mathfrak{L}(c)$, of $c$ in $\mathfrak{B}_{n, \varepsilon}$ with the same $\varrho$. As $\mathfrak{B}_{n, \varepsilon}$ is paracompact (Proposition B.1.2 of [9]), there exists a locally finite open cover, $\left\{\mathfrak{L}\left(c_{i}\right): i \in \Lambda\right\}$ together with a subordinate partition of unity, $\left\{\alpha_{i}: i \in \Lambda\right\}$ such that if $c \in \mathfrak{L}\left(c_{i}\right)$, Eq. (E.1.6) holds for all $\varrho_{i}<r<\infty$. Let $\varrho(c)=2 \sum_{i \in A} \alpha_{i}(c) \varrho_{i}$. Then $\varrho(c)$ $>2 \min \left\{\varrho_{i}: \alpha_{i}(c)>0\right\}$ and Eq. (E.1.7) holds.

Now, let $\delta \in C^{0}\left(\mathfrak{B}_{n, \varepsilon / 2} ;(0,1)\right)$ be given by Lemma E.1.7 and let $\varrho \in C^{0}\left(\mathfrak{B}_{n, \varepsilon / 2} ;[0, \infty)\right)$ be constructed from $\frac{1}{2} \delta$ as described by Lemma E.1.8. Then, Lemma E.1.7 constructs for each $c \in \mathfrak{B}_{n, \varepsilon / 2}$,

$$
\eta(c)=\tau_{c}\left(0,\left(1-\beta_{\varrho(c)}\right) G(c)\right) \in L^{2}(Q) \cap \Gamma(Q)
$$

to satisfy $G\left(c+\overline{\mathfrak{D}}_{c} \eta(c)\right)=\beta_{\varrho(c)} G(c)$. Here, $\beta_{\varrho} \in C_{0}^{\infty}\left(\mathbb{R}^{3} ;[0,1]\right)$ is as specified in Sect. A.3.

Lemma E.1.9. Let $\varepsilon$ be as in Lemma E.1.5. For $c \in \mathfrak{B}_{n, \varepsilon / 2}$, define $\eta(c)$ by Eq. (E.1.8). The assignment of $(t, c) \in[0,1] \times \mathfrak{B}_{n, \varepsilon / 2}$ to $\Pi\left(c+t \overline{\mathfrak{D}}_{c} \eta(c)\right) \in \mathfrak{B}$ defines a continuous map.

Proof of Lemma E.1.9. Proposition B.6.1 of [9] provides an open neighborhood, $\mathfrak{N}(c)$, of each $c \in \mathfrak{B}$ with a continuous $h[c]: \mathfrak{N}(c) \rightarrow \mathfrak{G}_{0}$ such that the assignment of $b \in \mathfrak{N}(c)$ to $m_{c}(b)=h(b) \cdot b-c$ embeds $\mathfrak{N}(c)$ in $\Gamma^{c}$ sending $c$ to $0 \in \Gamma^{c}$. The $\mathfrak{G}$ equivariance implies that for $b \in \mathfrak{N}(c)$,

$$
G\left(c+m_{c}(b)+\overline{\mathfrak{D}}_{c+m_{c}(b)} h[c](b) \cdot \eta(b)\right)=\beta_{\varrho(b)} G\left(c+m_{c}(b)\right) .
$$

Further, Lemma E.1.7 asserts that

$$
\|h[c](b) \cdot \eta(b)\|_{2} \leqq z v(b)^{-2}\left\|\left(1-\beta_{\varrho(b)}\right) G\left(c+m_{c}(b)\right)\right\|_{2} .
$$


On the other hand, Lemma E.1.7 and E.1.8 provides a neighborhood $\mathfrak{D} \subset \bar{\Gamma}^{c}$ of $0 \in \bar{\Gamma}^{c}$ and a unique, continuous $\xi: \mathfrak{D} \rightarrow L^{2}(Q) \cap \Gamma(Q)$ such that for $\phi \in \mathfrak{D}$,

$$
G\left(c+\phi+\overline{\mathfrak{D}}_{c+\phi} \xi(\phi)\right)=\beta_{\varrho(\Pi(c+\phi))} G(c+\phi),
$$

and

$$
\|\xi(\phi)\|_{2} \leqq z v(\Pi(c+\phi))^{-2}\left\|\left(1-\beta_{\varrho(\Pi(c+\phi))}\right) G(c+\phi)\right\|_{2} .
$$

As $\xi$ is unique, it must be true that on $\mathfrak{D} \cap m_{c}(\mathfrak{N}(c)), \xi\left(m_{c}(b)\right)=h[c](b) \eta(b)$. Thus, because $m_{c}$ is an imbedding, the assignment of $b \in \mathfrak{N}_{c}(b) \cap m_{c}^{-1}(\mathfrak{D})$ to $h[c](b) \eta(b)$ $\in L^{2}(Q) \cap \Gamma(Q)$ is continuous. As

$$
\Pi\left(c+m_{c}(b)+\overline{\mathfrak{D}}_{c+m_{c}(b)} h[c](b) \eta(b)\right)=\Pi\left(b+\overline{\mathfrak{D}}_{b} \eta(b)\right),
$$

one obtains, finally, Lemma E.1.9.

The proof of Lemma E.1.3 is completed by defining $m_{1}: \hat{\mathfrak{B}}_{n} \rightarrow \hat{\mathfrak{B}}_{n}$ as follows:

$$
m_{1}(t, c)=g(t, c) \Pi\left[c+t \beta\left(2 \varepsilon^{-1}(\mathfrak{A}(c)-4 \pi n)\right) \eta(c)\right],
$$

where $g:[0,1] \times \hat{\mathfrak{B}} \rightarrow\left(\mathfrak{G}\right.$ is provided by Lemma B.1.4, and $\beta \in C_{0}^{\infty}([0, \infty) ;[0,1])$ is a smooth function which is identically 1 on $\left[0, \frac{1}{2}\right]$ and identically 0 on $\left[\frac{3}{4}, \infty\right]$. Define the function $R$ by assigning $c \in \hat{\mathfrak{B}}_{n}$ to

$$
R(c) \equiv \varrho(c) \beta\left(2 \varepsilon^{-1}(\mathfrak{U}(c)-4 \pi n)\right)+1 .
$$

Proof of Lemma E.1.4. The lemma is proved by taking $\varepsilon$ as in Lemma E.1.5 and constructing for each $c \in \mathfrak{E}_{n, \varepsilon / 2} \equiv\left\{c \in \mathfrak{E}_{n}: \mathfrak{A}(c)<4 \pi n+\varepsilon / 2\right\}$ a map $\alpha_{c}:[0, \infty] \rightarrow \Gamma^{c}$ such that $\left\|G\left(c+\alpha_{c}(\cdot)\right)\right\|_{2}$ is decreasing to zero. Here, $[0, \infty]$ is topologized via the map $t \rightarrow 1-e^{-t}$ into $[0,1]$. The continuity of the assignment of $(t, c)$ to $\alpha_{c}(t)$ is proved using the local embeddings of Proposition B.6.1 of [9].

To begin the construction, define the linear space $\Omega^{c}=\left\{\psi \in \Gamma^{c}\right.$ : $\left.\operatorname{Im} \mathfrak{D}_{c} \psi \in H_{c}(Q)^{*}\right\}$. The space $\Omega^{c}$ is naturally a Fréchet space with the topology from the map which sends $\psi \in \Omega^{c}$ to $\left(\psi, \operatorname{Im} \mathfrak{D}_{c} \psi\right) \in \Gamma^{c} \times H_{c}(Q)^{*}$. For $c \in \mathfrak{E}_{n, \varepsilon / 2}, G(c) \in L^{6 / 5}(Q)$, and so by Lemma A.4.1 of [9], $G(c) \in H_{c}(Q)^{*}$. Moreover, the assignment of $\psi \in H_{c}(Q)$ to $\{\psi, \psi\}$ of Eq. (E.1.3) defines, via the $L^{2}$-pairing, a smooth map $\psi \mapsto\langle\{\psi, \psi\}, \cdot\rangle \in H_{c}(Q)^{*}$ of $H_{c}(Q)$ into $H_{c}(Q)^{*}$. Therefore, the assignment of $\psi \in \Omega^{c}$ to $G(c+\psi)=G(c)+\operatorname{Im} \mathfrak{D}_{c} \psi+\frac{1}{2}\{\psi, \psi\}$ defines a smooth map of $\Omega^{c}$ into $H_{c}(Q)^{*} \cap \Gamma(Q)$.

With $\varepsilon$ as in Lemma E.1.5 and for $c \in \mathfrak{E}_{n, \varepsilon / 2}$, define $\bar{\Omega}^{c}=\left\{\psi \in \Omega^{c}: \mathfrak{U}(c+\psi)\right.$ $<4 \pi n+\varepsilon\}$. Note that $\bar{\Omega}^{c}$ is open in $\Omega^{c}$.

Lemma E.1.10. Let $\varepsilon$ be as in Lemma E.1.5 and let $c \in \mathbb{E}_{n, \varepsilon / 2}$. For each $\psi \in \bar{\Omega}^{c}$, there exists a unique $u_{c}(\psi) \in \Gamma^{c}$ with the following properties:

(1) $\mathfrak{D}_{c+\psi} \mathfrak{D}_{c+\psi}^{*} u_{c}(\psi)=-G(c+\psi)$,

(2) $\left\|u_{c}(\psi)\right\|_{c+\psi} \leqq z\left(\|G(c+\psi)\|_{2}+\|G(c+\psi)\|_{* c+\psi}\right)$,

(3) $\left\|\mathfrak{D}_{c+\psi}^{*} u_{c}(\psi)\right\|_{c+\psi} \leqq z\left(\|G(c+\psi)\|_{2}+\|G(c+\psi)\|_{* c+\psi}\right)$,

(4) $\mathfrak{D}_{c+\psi}^{*} u_{c}(\psi) \in \Omega_{c}$.

Here, $\|\cdot\|_{* c+\psi}$ is the dual norm on $H_{c+\psi}(Q)^{*}$. The constant $z<\infty$ is independent of $c \in \mathbb{E}_{n, \varepsilon / 2}$ and $\psi \in \bar{\Omega}^{c}$. In addition, the assignments of $\psi \in \bar{\Omega}^{c}$ to $u_{c}(\psi) \in \Gamma^{c}$ and to $\mathfrak{D}_{c+\psi}^{*} u_{c}(\psi) \in \Omega_{c}$ define smooth maps. 
Proof of Lemma E.1.10. Define a map $T: \bar{\Omega}^{c} \times \Gamma^{c} \rightarrow \bar{\Omega}^{c} \times\left(H_{c}(Q)^{*} \cap \Gamma(Q)\right)$ by sending $(\psi, u)$ to $T(\psi, u)=\left(\psi, \mathfrak{D}_{c+\psi} \mathfrak{D}_{c+\psi}^{*} u+G(c+\psi)\right)$. According to Lemma E.1.6, this is a smooth map. Lemma E.1.5 asserts that the differential of $T$ at $(\psi, u)$ $\in \bar{\Omega}^{c} \times \Gamma^{c}$ is surjective, and therefore, Hamilton's inverse function theorem [28] provides a smooth map $u_{c}: \bar{\Omega}^{c} \rightarrow \Gamma^{c}$ satisfying Assertion (1) of Lemma E.1.10. Assertion (2) follows from Assertion (1) and Lemma E.1.5. Assertion (3) follows from Assertions (1), (2) and the Weitzenbock formula for $\mathfrak{D}_{c+\psi}$ in Eq. (6.13) of [8]. The calculations are straightforward and the interested reader can refer to Sect. B.3 of [9], where similar estimates are derived. Assertion (4) is a direct consequence of Assertion (1). The differentiability of the assignment of $\psi \in \bar{\Omega}^{c}$ to $\mathfrak{D}_{c+\psi}^{*} u_{c}(\psi) \in \Omega^{c}$ is also a straightforward calculation which is left to the reader.

The tangent space to $\bar{\Omega}^{c}$ is $\bar{\Omega}_{c} \times \Omega_{c}$ and thus, the assignment of $\psi \in \bar{\Omega}_{c}$ to $\mathfrak{D}_{c+\psi}^{*} u_{c}(\psi) \in \Omega_{c}$ defines a smooth vector field, $v$, on $\bar{\Omega}_{c}$. The results in [28] imply that the integral curves of $v$ exist and are unique. The following lemma summarizes:

Lemma E.1.11. Let $\varepsilon$ be as in Lemma E.1.5 and let $c \in \mathbb{E}_{n, \varepsilon / 2}$. For each $\psi \in \bar{\Omega}_{c}$, there exists a neighborhood of $\psi, \mathfrak{D}(\psi) \subset \bar{\Omega}^{c} ;$ a number, $t(\psi)>0$, and a unique, smooth map $\alpha_{c}:[0, t(\psi)) \times \mathfrak{O}(\psi) \rightarrow \bar{\Omega}^{c}$ such that for any fixed $\eta \in \mathfrak{D}(\psi)$, and for each $s \in(0, t(\psi))$,

$$
\left(\frac{d \alpha_{c}}{d t}\right)(s, \eta)=\left(\mathfrak{D}^{*} u_{c}\right)\left(\alpha_{c}(s, \eta)\right) \text { and } \alpha_{c}(0, \eta)=\eta \text {. }
$$

This lemma asserts that the integral curves of $\alpha$ exist for short times. The next lemma extends existence to $[0, \infty]$.

Lemma E.1.12. Let $\varepsilon$ be as in Lemma E.1.5 and let $c \in \mathfrak{E}_{n, \varepsilon / 2}$. There exists a neighborhood $\mathfrak{D}$ of $o \in \bar{\Omega}^{c}$ such that $\alpha_{c}$ of Eq. (E.1.10) has a unique extension for which the assignment of $(s, \eta) \in[0,1] \times \mathfrak{D}$ to $\alpha_{c}(-\ln (1-s), \eta)$ defines a continuous map from $[0,1] \times \mathfrak{D}$ to $\bar{\Omega}^{c}$.

Proof of Lemma E.1.12. Lemma E.1.11 implies that if $\psi \in \bar{\Omega}^{c}$ and if $\eta \in \mathfrak{D}(\psi)$ and $s \in[0, t(\psi))$, then for $\alpha \equiv \alpha_{c}$,

$$
\left(\frac{d G}{d s}\right)(c+\alpha(s, \eta))=-G(c+\alpha(s, \eta)) .
$$

Therefore, as long as $s \in[0, t(\psi))$,

$$
G(c+\alpha(s, \eta))=e^{-s} G(c+\eta),
$$

and

$$
\|G(c+\alpha(s, \eta))\|_{2}=e^{-s}\|G(c)\|_{2} .
$$

The first step for Lemma E.1.12 is

Lemma E.1.13. Let $\varepsilon$ and $c \in \mathfrak{E}_{n, \varepsilon / 2}$ be as in Lemma E.1.12, and let $\alpha_{c}$ be the map of Lemma E.1.11. Then $\alpha_{c}(-\ln (1-s), 0)$ is well defined for all $s \in[0,1]$ and the assignment of $s$ to $\alpha_{c}(-\ln (1-s), 0)$ defines a continuous map from $[0,1]$ into $\bar{\Omega}_{c}$.

Proof of Lemma E.1.13. According to Lemma E.1.11, $\alpha(t, 0)$ exists for $t \in[0, t(0))$ with $t(0)>0$. Suppose that $\alpha(\cdot, 0)$ has been extended to a continuous map from 
$[0, T)$ to $\bar{\Omega}_{c}$ for some $T \leqq \infty$. Then by showing that it extends uniquely to a continuous map from $[0, T]$ to $\bar{\Omega}_{c}$, one could then infer Lemma E.1.13 from Lemma E.1.11. Now, note that if $c \in \mathfrak{E}_{n, \varepsilon / 2}$, then $G(c) \in L^{6 / 5}(Q)$ and, therefore, if $s \in[0, T)$, Eq. (E.1.12) implies

$$
\|G(c+\alpha(s, 0))\|_{6 / 5}=e^{-s}\|G(c)\|_{6 / 5} .
$$

Lemma A.4.1 of [9] implies that $L^{6 / 5}(Q)$ embeds in $H_{c}(Q)^{*}$ with embedding constant $z<\infty$, independent of $c \in \mathbb{C}$. Thus,

$$
\|G(c+\alpha(s, 0))\|_{* c+\alpha(s, 0)} \leqq z e^{-s}\|G(c)\|_{6 / 5} .
$$

For any $\psi \in \bar{\Omega}_{c}$, and $s \in(0, t(\psi))$, Proposition B.6.2 of [9] implies that if $b=c+\alpha(s, \psi)$, then

$$
\left|\frac{d}{d s}\|\alpha(s, \psi)\|_{b}\right| \leqq z \cdot\left\|\frac{d \alpha}{d s}(s, \psi)\right\|_{b}\left(1+\|\alpha(s, \psi)\|_{b}\right),
$$

where $z<\infty$ is independent of $c, \psi$, and $s$. In the present predicament, Eqs. $($ E.1.13, 5, 6) and Lemma E.1.10 imply that

$$
\left|\frac{d}{d s} \ln \left(1+\|\alpha(s, 0)\|_{b}\right)\right|<z e^{-s}\left(1+\|G(c)\|_{6 / 5}\right),
$$

and so, by integrating, that

$$
\|\alpha(s, 0)\|_{c+\alpha(s, 0)}<\xi(c),
$$

where $\xi(c)$ depends on $c$ only through $\|G(c)\|_{6 / 5}$. Via Proposition B.6.2 of [9], this last equation also asserts that with a different $\xi(c)$,

$$
\|\alpha(s, 0)\|_{c}<\xi(c) \text {. }
$$

Now, if $[s, t] \in[0, T)$, then

$$
\begin{aligned}
\|\alpha(t, 0)-\alpha(s, 0)\|_{c} & \leqq \int_{s}^{t} d \tau\left\|\frac{d \alpha}{d \tau}(\tau, 0)\right\|_{c} \\
& \leqq \int_{s}^{t} d \tau\left\|\frac{d \alpha}{d \tau}(\tau, 0)\right\|_{c+\alpha(\tau, 0)}(1+\xi(c)), \\
& <e^{-s} z \xi(c),
\end{aligned}
$$

with a different $\xi(c)$. The second line, above, follows from the first using Eq. (E.1.7) and Proposition B.6.2 of [9]. The third line uses Lemma E.1.10 and Eqs. (E.1.13, 15). Equation (E.1.19) implies that $\{\alpha(s, 0): s \in[0, T)\}$ converges strongly in $H_{c}(Q)$ as $s \rightarrow T$. By bootstrapping, one obtains the convergence of $\{\alpha(s, 0)\}$ in $\Gamma^{c}$ as well (cf. [28]). Equation (E.1.13) implies that this limit, $\alpha(T, 0)$, is in $\bar{\Gamma}^{c}$. Further, Eq. (E.1.15) implies that $\{G(c+\alpha(s, 0))\}$ converges strongly in $L^{6 / 5}(Q)$ to a limit which must a priori be $G(c+\alpha(T, 0))$. Therefore, $\{G(c+\alpha(s, 0))\}$ converges strongly in $H_{c}(Q)^{*}$. This last fact, with the convergence of $\{\alpha(s, 0)\}$ in $H_{c}(Q)$ implies that $\mathfrak{D}_{c+\alpha(s, 0)} \alpha(s, 0)$ converges strongly in $H_{c}(Q)^{*}$, too. The conclusion is that $\{\alpha(s, 0): s \in[0, T)\}$ converges to $\alpha(T, 0)$ in $\bar{\Omega}_{c}$. 
The second step in the proof of Lemma E.1.13 is to obtain the existence of $\alpha$ of Lemma E.1.11 on $[0, \infty] \times \mathfrak{O}$ for some neighborhood $\mathfrak{O}$ of $o \in \bar{\Omega}_{c}$. For this purpose, suppose that $T<\infty$ exists with a neighborhood $\mathfrak{D}(T)$ of $o$ in $\bar{\Omega}_{c}$ such that $\alpha$ exists and is continuous on $[0, T) \times \mathfrak{D}$. By Lemma E.1.13, $\alpha(T, 0) \in \bar{\Omega}_{c}$ exists and there exists a neighborhood, $\mathfrak{D}^{\prime}$ of $\alpha(T, 0)$ in $\bar{\Omega}_{c}$ and $t>0$ such that $\alpha$ exists and is continuous on $[0, t) \times \mathfrak{D}^{\prime}$. By Lemma E.1.13, there exists $s \in\left[T-\frac{1}{2} t, T\right)$ such that $\alpha(s, 0) \in \mathfrak{D}^{\prime}$ and, therefore, there exists a neighborhood, $\mathfrak{D}^{\prime \prime} \subseteq \mathfrak{D}$ such that $\alpha(s, \eta) \in \mathfrak{D}^{\prime}$ for all $\eta \in \mathfrak{D}^{\prime \prime}$. Then by the uniqueness of assertion of Lemma E.1.11, $\alpha$ extends to $\operatorname{map}\left[0, T+\frac{t}{2}\right] \times \mathfrak{D}^{\prime \prime}$ into $\bar{\Omega}_{c}$ continuously. The conclusion is the following: Given $T<\infty$, there exists a neighborhood $\mathfrak{O}(T)$ of $o$ in $\bar{\Omega}_{c}$ such that $\alpha:[0, T) \times \mathfrak{O}(T) \rightarrow \bar{\Omega}_{c}$ continuously. Further, since $\{\alpha(t, 0): t>0\}$ converges to $\alpha(\infty, 0) \in \bar{\Omega}_{c}$, one can conclude that given a neighborhood, $Q$, of $\alpha(\infty, 0)$ in $\bar{\Omega}_{c}$, there exists $T<\infty$ and a neighborhood $\mathfrak{D}(Q) \subset \mathfrak{D}(T)$ of $o$ in $\bar{\Omega}_{c}$, such that for all $\eta \in \mathfrak{D}(Q), \alpha(T, \eta) \in Q$. Thus, Lemma E.1.12 is a consequence of the uniqueness assertion of Lemma E.1.11 together with

Lemma E.1.14. Let $\varepsilon$ and $c$ be as in Lemma E.1.12. Let $\alpha(\infty, 0) \in \bar{\Omega}_{c}$ be as in Lemma E.1.13. There exists a neighborhood $Q$ of $\alpha(\infty, 0)$ in $\bar{\Omega}_{c}$ such that $\alpha$ maps $[0, \infty] \times Q$ continuously into $\bar{\Omega}_{c}$.

Proof of Lemma E.1.14. As $G(c+\alpha(\infty, 0))=0$, there exists for each $\delta>0$, a convex neighborhood $Q(\delta)$ of $\alpha(\infty, 0)$ in $\bar{\Omega}_{c}$ such that for all $\eta \in Q(\delta)$,

$$
\|G(c+\eta)\|_{2}+\|G(c+\eta)\|_{* c+\eta}<\delta .
$$

Lemma E.1.11 insures that no generality is lost by assuming that $\alpha$ exists and is continuous on $[0, t) \times Q(\delta)$ for some $t \in(0, \infty)$. For $\delta>0$, but sufficiently small, $\alpha$ will be shown to extend to $[0, \infty] \times Q(\delta)$. To begin, observe that Proposition B.6.2 of [9] implies that $z<\infty$ exists such that for any $c \in \mathfrak{C}, \psi \in H_{c}(Q)$, and $G \in H_{c}(Q)^{*}$,

$$
\|G\|_{* c+\psi} \leqq z\|G\|_{* c}\left(1+\|\psi\|_{* c+\psi}\right) .
$$

Thus, for any $(s, \eta) \in[0, t) \times Q(\delta)$, Eqs. $($ E.1.13, 21) imply that

$$
\|G(c+\alpha(s, \eta))\|_{* c+\alpha(s, \eta)} \leqq z e^{-s}\|G(c+\eta)\|_{* c+\eta}\left(1+\|\alpha(s, \eta)-\eta\|_{c+\eta}\right),
$$

and thus, with Eq. (E.1.20),

$$
\|G(c+\alpha(s, \eta))\|_{* c+\alpha(s, \eta)}<z \delta e^{-s}\left(1+\|\alpha(s, \eta)-\eta\|_{c+\alpha(s, \eta)}\right) .
$$

Now, Eqs. (E.1.13, 16, 22) and Lemma E.1.10 imply that

$$
\left|\frac{d}{d s}\|\alpha(s, \eta)-\eta\|_{c+\alpha(s, \eta)}\right|<z \delta e^{-s}\left(1+\|\alpha(s, \eta)-\eta\|_{c+\alpha(s, \eta)}^{2}\right) .
$$

The integral form of the preceding inequality asserts that if $(s, \eta) \in[0, t) \times Q(\delta)$, then

$$
\|\alpha(s, \eta)-\eta\|_{c+\alpha(s, \eta)}<\frac{z \delta\left(1-e^{-s}\right)}{1-z \delta\left(1-e^{-s}\right)} .
$$


Here, the reader should check that the constant $z$ is independent of $c, \eta, s$, and $\delta$. Equation (E.1.24) implies that if $\delta<\frac{1}{2} z^{-1} \equiv \delta_{0}$, then

$$
\|\alpha(s, \eta)-\eta\|_{c+\alpha(s, \eta)}<1
$$

and therefore, $\|\alpha(s, \eta)-\eta\|_{c+\eta}$ is uniformly bounded on $[0, t) \times Q(\delta)$ if $\delta<\delta_{0}$.

Next, observe that if $[r, s] \subset[0, t)$, and if $\eta \in Q(\delta)$, then

$$
\begin{aligned}
\|\alpha(s, \eta)-\alpha(r, \eta)\|_{c+\eta} & \leqq \int_{r}^{s} d \tau\left\|\frac{d \alpha}{d \tau}(\tau, \eta)\right\|_{c+\eta}, \\
& \leqq 2 z \int_{r}^{s} d \tau\left\|\frac{d \alpha}{d \tau}(\tau, \eta)\right\|_{c+\alpha(\tau, \eta)}, \\
& \leqq 2 z \delta e^{-r} .
\end{aligned}
$$

Here, Line 2 follows from Line 1 with Eq. (E.1.25) and Proposition B.6.2 of [9], while Line 3 follows from Line 2 with Eqs. (E.1.13, 22, 25) and Lemma E.1.10. Equation (E.1.26) asserts that the sequence $\{\alpha(s, \eta): s \in[0, t)\}$ for fixed $\eta \in Q(\delta)$ is Cauchy in $H_{c}(Q)$ and that it converges uniformly, with respect to $\eta \in Q(\delta)$ to some $\alpha(t, \eta) \in Q(\delta)$. By bootstrapping as in the proof of Lemma E.1.13, one obtains the uniform convergence on $Q(\delta)$ of $\{\alpha(s, \eta): s \in[0, t)\}$ in $\bar{\Omega}^{c}$ to $\alpha(t, \eta)$. The uniform convergence implies that $\alpha$ extends continuously to a map from $[0, t] \times Q(\delta)$ to $\bar{\Omega}^{c}$ as long as $\delta<\delta_{0}$. This result, with the local existence assertion of Lemma E.1.11 implies Lemma E.1.14.

Completion of the proof of Lemma E.1.4. Let $\beta$ be a function in $C_{0}^{\infty}([0, \infty) ;[0,1])$ which is identically 1 on $\left[0, \frac{1}{2}\right]$ and identically zero on $\left[\frac{3}{4}, \infty\right)$. Let $\Pi: \mathbb{C} \rightarrow \mathfrak{B}$ be the projection. Let $\varepsilon$ be as in Lemma E.1.5 and let $\alpha$ be as in Lemma E.1.12. Then define $\mathrm{m}_{2}:[0,1] \times \hat{\mathfrak{E}}_{n} \rightarrow \hat{\mathfrak{E}}_{n}$ by setting

$$
\mathfrak{m}_{2}(t, c)=g(t, c) \Pi\left[c+\beta\left(2 \varepsilon^{-1}(\mathfrak{U}(c)-4 \pi n)\right) \alpha_{c}(-\ln (1-t), 0)\right] .
$$

Here, $\alpha_{c}$ is as specified by Lemmas E.1.11 and E.1.12 and $g \in C^{0}\left(\hat{\mathfrak{E}}_{n} ; \mathfrak{5}\right)$ is constructed by replacing $\mathfrak{B}_{n}$ by $\mathfrak{E}_{n}$ in the proof of Lemma B.1.4. Equation (E.1.12) insures that $\mathrm{m}_{2}$ satisfies Assertions (1) and (2) of Lemma E.1.4. The only remaining issue is the question of continuity. This is a local question; and as with the map $m_{1}$ of Lemma E.1.3, the continuity question is settled by exploiting the local embeddings of Proposition B.6.1 of [9]. Let $c \in \hat{\mathfrak{E}}_{n}$ and let $\mathfrak{N}(c)$ be the open neighborhood of $c$ in $\mathfrak{B}_{n}$ with continuous $h_{c}: \mathfrak{N}(c) \rightarrow \mathfrak{G}_{0}$ as provided by Proposition B.6.1 of [9]. Thus, the map sending $b \in \mathfrak{N}(c)$ to $m_{c}(b)=h_{c}(b) \cdot b-c$ embeds $\mathfrak{N}(c)$ in $\Gamma^{c}$ sending $c$ to $o \in \Gamma^{c}$. If it can be established that $m$ sends $\mathfrak{N}(c) \cap \hat{\mathfrak{E}}_{n}$ continuously into $\Gamma^{c}$, then the uniqueness and continuity of $\alpha_{c}$ on $[0, \infty] \times\{$ Open neighborhood of $o$ in $\left.\Omega^{c}\right\}$ implies the continuity of $m_{2}$. The argument is the same as for the proof of continuity of $m_{1}$ in Lemma E.1.3. Thus, continuity of $m_{2}$ follows by showing that the assignment of $b \in \mathfrak{N}(c) \cap \hat{\mathfrak{E}}_{n}$ to $\operatorname{Im} \mathfrak{D}_{c} m_{c}(b) \in H_{c}(Q) *$ is continuous. For this, note that the assignment of $b \in \mathfrak{N}(c) \cap \hat{\mathbb{E}}_{n}$ to $G\left(c+m_{c}(b)\right) \in L^{6 / 5}(Q)$ is continuous (use Lemma A.4.6 of [9]), so by Lemma A.4.1 of [9], the assignment of $b \in \mathfrak{N}(c) \cap \hat{\mathfrak{E}}_{n}$ to $G\left(c+m_{c}(b)\right)$ defines a continuous map into $H_{c}(Q)^{*}$. Further, the assignment of $\psi=(a, \phi) \in H_{c}(Q)$ to $\frac{1}{2}\{\psi, \psi\}$ of Eq. (E.1.3) defines, via the $L^{2}$-pairing, a continuous map from $H_{c}(Q)$ into $H_{c}(Q)^{*}$. Therefore, Eq. (E.1.13) implies that $\operatorname{Im} \mathfrak{D}_{c} m_{c}(b)$ defines a continuous map from $\mathfrak{N}(c) \cap \hat{\mathfrak{E}}_{n}$ into $H_{c}(Q)^{*}$ as required. 


\section{E.2. Min-Max and Pointed Homotopy}

The purpose of this section is to complete the proofs of Theorems A.1.2 and A.1.4. Theorem A.1.2 is observed to be a direct consequence of Proposition C.1.1 and Corollary E.1.2. To prove Theorem A.1.4, one is considering only families of subsets of $\hat{\mathfrak{B}}_{n}$ which are invariant under homotopies which fix a given subset of $\hat{\mathfrak{B}}_{n}$. For a given $n \in \mathbb{Z}$ and $l=\{0, \ldots\}$, let $\phi \in C^{0}\left(S^{l} ; \hat{\mathfrak{M}}_{n}\right)$. The proof of Theorem A.1.4 requires answering the question of when a given path component $\Theta \in\left\{\psi \in C^{0}\left(B^{l+1} ; \hat{\mathfrak{B}}_{n}\right):\left.\psi\right|_{\partial B^{l+1}}=\phi\right\}$ contains a map whose image lies in $\hat{\mathfrak{M}}_{n}$. Here, $B^{l+1}$ is the standard unit ball on $\mathbb{R}^{l+1}$ with boundary $S^{l}$. To prove Theorem A.1.4 by min-max arguments, define for each such $\Theta$, the number

$$
\mathfrak{A}_{\Theta}=\inf _{\psi \in \Theta} \sup _{y \in B^{1+1}} \mathfrak{Y}(\psi(y)) .
$$

The min-max theory for $\Theta$ results in

Proposition E.2.1. Let $n \in \mathbb{Z}$, let $l \in\{0,1, \ldots\}$ and let $\phi \in C^{0}\left(S^{l} ; \hat{\mathfrak{M}}_{n}\right)$ be given. Let $\Theta$ be a path component of $\left\{\psi \in C^{0}\left(B^{l+1} ; \hat{\mathfrak{B}}_{n}\right):\left.\psi\right|_{S^{l}}=\phi\right\}$. There exists a critical point $c(\Theta) \in \hat{\mathfrak{B}}_{n}$ of $\mathfrak{A}$ with critical value $\mathfrak{\mathfrak { A }}_{\Theta}$ and with $\gamma_{c(\Theta)}(l+2)$ of Definition A.4.4 non-negative.

Proof of Theorem A.1.4, assuming Proposition E.2.2. Fix $n \in \mathbb{Z}$ and $l \in\{0,1,2, \ldots\}$. Take $\phi$ to map $S^{l}$ to a point, $* \in \hat{\mathfrak{M}}_{n}$. Then a path component $\Theta \cong\left\{\psi \in \mathrm{C}^{0}\left(\mathbf{B}^{l+1} ; \hat{\mathfrak{B}}_{n}\right):\left.\psi\right|_{S^{l}}=*\right\}$ is precisely an element in $\pi_{l+1}\left(\hat{\mathfrak{B}}_{n}\right)$. Theorem 1.2 of [8] asserts that if a critical point $c \in \hat{\mathfrak{B}}_{n}$ of $\mathfrak{A}$ is not in $\hat{\mathfrak{M}}_{n}$, then $\gamma_{c}(|n|+1)<0$. So if $l+1<|n|$ and if $\Theta \in \pi_{l+1}\left(\hat{\mathfrak{B}}_{n}\right)$, then Proposition E.2.1 implies that $c(\Theta) \in \hat{\mathfrak{M}}_{n}$ and $\mathfrak{A}(c(\Theta))=\mathfrak{A}_{\Theta}$. Proposition E.1.1 implies that $\psi \in \Theta$ exists with $\operatorname{Im} \psi \subseteq \mathfrak{M}_{n}$. Thus, the inclusion homomorphism $\pi_{k}\left(\hat{\mathfrak{M}}_{n}\right) \rightarrow \pi_{k}\left(\hat{\mathfrak{B}}_{n}\right)$ is surjective for $k \leqq|n|$. Next, let $Z_{k, n}$ denote the kernel of this homomorphism. For $k<|n|$, let $[\phi] \in Z_{k, n}$ be represented by $\phi \in C^{0}\left(S^{k} ; \hat{\mathfrak{M}}_{n}\right)$. A map $\psi \in C^{0}\left(B^{l+1} ; \hat{\mathfrak{B}}^{n}\right)$ with $\left.\psi\right|_{S^{l}}=\phi$ is a homotopy of $\phi$ to the constant map. By Propositions E.1.1, E.2.1 and Theorem 1.2 of [8], there exists such a $\psi$ with $\operatorname{Im} \psi \subseteq \mathfrak{M}_{n}$; so for $k<|n|$, the inclusion homomorphism $\pi_{k}\left(\hat{\mathfrak{M}}_{n}\right)$ $\rightarrow \pi_{k}\left(\hat{\mathfrak{B}}_{n}\right)$ is injective.

Proof of Proposition E.2.1. The proof is essentially the proof of Theorem A.1.2 save for two modifications. The first modification is to take into account that for a given $\phi$, each $\Theta$ does not define a homotopy invariant family of subsets of $\hat{\mathfrak{B}}_{n}$, but a family of compact subsets which is invariant under homotopies which restrict to $\operatorname{Im} \phi$ as the identity for all $t$. The second modification is for obtaining the a priori estimate on $\gamma(l+2)$. This is a straightforward application of the construction in Sect. 5 of [22] to the present situation. As both modifications are uncomplicated, the discussion will be brief.

Fix $n \in \mathbb{Z}$ and let $\varepsilon>0$ and $\mathfrak{m}:[0,1] \times \hat{\mathfrak{B}}_{n} \rightarrow \hat{\mathfrak{B}}_{n}$ be as in Proposition E.1.1. Proposition B.5.2 of [9] provides a continuous homotopy $q\left(\frac{\varepsilon}{2}, \cdot, \cdot\right):[0,1]$ $\times \hat{\mathfrak{B}} \rightarrow \hat{\mathfrak{B}}$ such that $q\left(\frac{\varepsilon}{2}, 1, \cdot\right): \hat{\mathfrak{B}}_{n} \rightarrow \hat{\mathfrak{B}}_{n}^{\delta}$ continuously for all $\delta \in\left[0, \frac{1}{2}\right)$ and such that for all $t \in[0,1]$, and $y \in S^{l}$

$$
\mathfrak{A}\left(q\left(\frac{\varepsilon}{2}, t, \phi(y)\right)\right)<4 \pi|n|+\frac{\varepsilon}{2} .
$$


For each $\delta \in\left[0, \frac{1}{2}\right)$, let $\hat{\phi}=q\left(\frac{\varepsilon}{2}, 1, \phi(\cdot)\right) \in C^{0}\left(S^{l} ; \hat{\mathfrak{B}}_{n}^{\delta}\right)$. Let

$$
\Theta \subseteq\left\{\psi \in C^{0}\left(B^{l+1} ; \hat{\mathfrak{B}}_{n}\right):\left.\psi\right|_{S^{l}}=\phi\right\}
$$

be a path component. Then $\Theta$ defines a path component, $\Theta^{\delta}$, of $\left\{\psi \in C^{0}\left(B^{l+1} ; \hat{\mathfrak{B}}_{n}^{\delta}\right\}:\left.\psi\right|_{S^{l}}=\hat{\phi}\right\}$ by assigning to each $\psi \in \Theta, \hat{\psi}=q\left(\frac{\varepsilon}{2}, 1, \psi(\cdot)\right)$. Notice that if $\psi_{1} \sim \psi_{2}\left(\operatorname{rel} S^{l}\right)$ then $\hat{\psi}_{1} \sim \hat{\psi}_{2}\left(\operatorname{rel} S^{l}\right)$. (Here " $\sim$ " means "homotopic to".) Conversely, each path component $\Theta^{\delta}$ of $\left\{\psi \in C^{0}\left(B^{l+1} ; \hat{\mathfrak{B}}_{n}^{\delta}\right)\right.$ : $\left.\left.\psi\right|_{S^{l}}=\hat{\phi}\right\} \quad$ defines a path component of $\left\{\psi \in C^{0}\left(B^{l+1} ; \hat{\mathfrak{B}}_{n}\right):\left.\psi\right|_{S^{l}}=\phi\right\}$ as follows: Let $t \in[0,1]$ denote the radial coordinate on $B^{l+1}$ and let $y$ denote a point in $S^{l}$. If $\psi \in \Theta^{\delta}$, define $\psi^{0}$ by

$$
\psi^{0}(t, y)=\left\{\begin{array}{lll}
\psi(2 t, y) & \text { if } & t \in\left[0, \frac{1}{2}\right] \\
q\left(\frac{\varepsilon}{2}, 2-2 t, \phi(y)\right) & \text { if } & t \in\left[\frac{1}{2}, 1\right] .
\end{array}\right.
$$

Notice that if $\psi_{1}, \psi_{2} \in \Theta^{\delta}$ and $\psi_{1} \sim \psi_{2}\left(\operatorname{rel} S^{l}\right)$, then $\psi_{1}^{0} \sim \psi_{2}^{0}\left(\operatorname{rel} S^{l}\right)$. Also, if $\psi \in \Theta$, then $(\hat{\psi})^{0} \sim \psi\left(\operatorname{rel} S^{l}\right)$. Conversely, if $\psi \in \Theta^{\delta}$, then $\left(\widehat{\psi^{0}}\right) \sim \psi\left(\operatorname{rel} S^{l}\right)$. Thus, the path components of $\left\{\psi \in C^{0}\left(B^{l+1} ; \hat{\mathfrak{B}}_{n}^{\delta}\right):\left.\psi\right|_{S^{l}}=\hat{\phi}\right\}$ and those of $\left\{\psi \in C^{0}\left(B^{l+1} ; \hat{\mathfrak{B}}_{n}\right):\left.\psi\right|_{S^{l}}=\phi\right\}$ are in $1-1$ correspondence.

Now, suppose that $\mathfrak{U}_{\Theta}>4 \pi|n|$. Then Proposition E.1.1 asserts that $\mathfrak{U}_{\Theta} \geqq 4 \pi|n|+\varepsilon$. For such $\Theta$, define

$$
\mathfrak{U}_{\Theta}^{\delta}=\inf _{\psi \in \Theta^{\delta}} \sup _{y \in B^{l+1}} \mathfrak{A}^{\delta}(\psi(y)) .
$$

Lemma E.2.2. As a function of $\delta \in\left[0, \frac{1}{2}\right), \mathfrak{H}_{\Theta}^{\delta}$ is non-decreasing and $\lim _{\delta \rightarrow 0} \mathfrak{U}_{\Theta}^{\delta}=\mathfrak{A}_{\Theta}$.

Proof of Lemma E.2.2. Mimic the proof of Lemma B.1.2 using the map $q$ : [0,1] $\times[0,1] \times \hat{\mathfrak{B}} \rightarrow \hat{\mathfrak{B}}$ of Proposition B.5.2 of [9]. Use the fact that because $\mathfrak{U}_{\Theta} \geqq 4 \pi|n|+\varepsilon$, for each $\phi \in \Theta$, there exists $\tau \in[0,1)$ such that if $x \in B^{l}$ and $|x|>\tau$, then $\mathfrak{A}(\psi(x))<4 \pi|n|+\varepsilon$. The details are left to the reader.

For $\Theta$ satisfying $\mathfrak{U}_{\Theta} \geqq 4 \pi|n|+\varepsilon$, and for each $\delta \in\left[0, \frac{1}{2}\right)$, let

$$
Y^{\delta}(\Theta)=\left\{(\psi, c) \in \Theta^{\delta} \times \hat{\mathfrak{B}}_{n}^{\delta}: c \in \operatorname{Im} \psi \text { and } \mathfrak{U}^{\delta}(c) \geqq \mathfrak{U}_{\Theta}^{\delta}\right\}
$$

For such $\Theta$, the analog of Proposition B.2.1 is

Proposition E.2.3. Let $\Theta$ be as described above with $\mathfrak{A}_{\Theta} \geqq 4 \pi|n|+\varepsilon$. There exists $\delta(\Theta) \in\left(0, \frac{1}{2}\right]$ such that given $\delta \in[0, \delta(\Theta))$ and $\varrho>0$, there are $(\psi, c) \in Y^{\delta}(\Theta)$ with

(1) $\max \left\{\mathfrak{U}^{\delta}(\psi(y)): y \in B^{l+1}\right\}<\mathfrak{U}_{\Theta}^{\delta}+\varrho$,

(2) $\left\|\nabla \mathfrak{U}_{c}^{\delta}\right\|_{*}<\varrho$,

(3) $\gamma_{c}^{\delta}(l+2)>-\varrho$,

(4) if $c=(A, \Phi)$, then $\nabla_{A}^{*} \nabla_{A} \Phi=0$ and $c$ also satisfies $E q$. (B.2.1).

Proof of Proposition E.2.1, given Proposition E.2.3. By mimicking the proof of Proposition B.1.3 (see Proposition B.3.1) one obtains for each $\delta \in(0, \delta(\Theta))$ a critical point $c^{\delta}(\Theta)$ of $\mathfrak{U}^{\delta}$ in $\hat{\mathfrak{B}}_{n}^{\delta}$ with critical value $\mathfrak{U}_{\Theta}^{\delta}$. This uses Assertions (1), (2), and (4) of Proposition E.2.3 to construct a good sequence with the properties that 
Proposition B.3.1 requires. The convergence assertion of Proposition B.3.1 and Assertion (3) of Proposition E.2.3 imply that $\gamma_{l+2}^{\delta}(c(\Theta)) \geqq 0$ (see Proposition 4.13 of [6, Part II] and its proof). Now, if $\mathfrak{U}(\Theta)<4 \pi|n|+\varepsilon$, Proposition E.1.1 implies Proposition E.2.1. If $\mathfrak{A}(\Theta)>4 \pi|n|+\varepsilon$, then by copying word for word the proof of Proposition C.1.1, one obtains the existence of a subsequence, $\left\{c_{i}\right\}_{i=1}^{\infty}$ $C\left\{c^{\delta}(\Theta): \delta \in(0, \delta(\Theta))\right\}$ which converges in $\hat{\mathfrak{B}}_{n}$ to a critical point $c(\Theta)$ of $\mathfrak{A}$ with critical value $\mathfrak{U}_{\Theta}$. This convergence implies that $\gamma_{l+2}(c(\Theta)) \geqq 0$ (see, again, Proposition 4.13 of [6, Part II]).

Proof of Proposition E.2.3. Since $\mathfrak{A}_{\Theta} \geqq 4 \pi|n|+\varepsilon$, there exists $\delta(\Theta) \in\left(0, \frac{1}{2}\right]$ such that for all $\delta<\delta(\Theta), \max \left\{\mathfrak{H}^{\delta}(\hat{\phi}(y)): y \in S^{l}\right\}<\mathfrak{H}_{\Theta}^{\delta}-\frac{\varepsilon}{4}$. For $\delta \in[0, \delta(\Theta))$ and a given $\varrho \in(0,1)$, the proof of Proposition B.2.1 is readily adapted to provide $(\psi, b) \in Y^{\delta}(\Theta)$ for which Assertions (1), (2), and (4) of Proposition E.2.3 hold. Indeed, the homotopies in Sect. B.2 can be readily altered so that each fixes the set $\left\{c \in \hat{\mathfrak{B}}_{n}^{\delta}\right.$ : $\left.\mathfrak{A}^{\delta}(c)<\mathfrak{A}_{\Theta}^{\delta}-\frac{\varepsilon}{8}\right\}$ for all $t \in[0,1]$; and hence fixes $\phi\left(S^{l}\right)$. In fact, the homotopies in Sect. 3 allow one to construct, given $\varrho>0$, a pair $(\psi, b) \in Y^{\delta}(\Theta)$ such that Assertions (1) and (2) are satisfied, and Assertion (4) is satisfied not just at $b$, but at all $c \in \operatorname{Im} \psi$ for which $\mathfrak{A}^{\delta}(c)>\mathfrak{U}_{\Theta}^{\delta}-\frac{\varepsilon}{16}$. That there exists $c \in \operatorname{Im} \psi$ with $\mathfrak{I}^{\delta}(c) \geqq \mathfrak{U}_{\Theta}^{\delta}$, and such that $\left\|\nabla \mathfrak{A}_{c}^{\delta}\right\|_{*}+\max \left(0,-\gamma_{c}^{\delta}(l+2)\right)$ are small $\left(0\left(\varrho^{1 / 3}\right)\right)$ is argued by showing that the alternative leads to a contradiction. Indeed, should this number be uniformly large on $\operatorname{Im} \psi \cap\left(\mathfrak{H}^{\delta}\right)^{-1}\left(\left[\mathfrak{H}_{\Theta}^{\delta}, \infty\right)\right)$ then the construction in Sect. 5 of [22] and specifically Lemmas 5.1-5.3 of [22] readily adapts to the present problem and would produce a map $\psi^{\prime} \in \Theta$ with $\max \left\{\mathfrak{Q}^{\delta}\left(\psi^{\prime}(y)\right): y \in B^{l+1}\right\}<\mathfrak{O}_{\Theta}^{\delta}$. The adaptation of Sect. 5 of [22] to the present circumstances is straightforward, given the uniform bounds in Proposition A.4.3 and given the continuity of $\gamma_{c}^{\delta}(l)$ as a function of $c \in \hat{\mathfrak{B}}^{\delta}$ (Proposition A.4.5).

\section{References}

1. Atiyah, M.F., Bott, R.: The Yang-Mills equations over Riemann surfaces. Philos. Trans. R. Soc. London A 308, 523 (1982)

2. Bott, R.: Morse theoretic aspects of Yang-Mills theory. In: Recent developments in gauge theories, Cargese, France. New York: Plenum Press 1980

3. Palais, R.: Critical point theory and mini-max principle. Proc. Symp. Pure Math., Vol. 15, Providence, RI: American Math Society 1970

4. Berger, M.: Nonlinearity and functional analysis. New York: Academic Press 1977

5. Woo, G.: Pseudo-particle configurations in two-dimensional fragments. J. Math. Phys. 18, 1264 (1977)

6. Taubes, C.H.: The existence of a non-minimal solution to the SU(2) Yang-Mills-Higgs equations on $\mathbb{R}^{3}$, Part I. Commun. Math. Phys. 86, 257 (1982); Part II. Commun. Math. Phys. 86, 299 (1982)

7. Groisser, D.: SU(2) Yang-Mills-Higgs theory on $\mathbb{R}^{3}$. Harvard University Ph.D. Thesis, 1983

8. Taubes, C.H.: Stability in Yang-Mills theories. Commun. Math. Phys. 91, 235 (1983)

9. Taubes, C.H.: Monopoles and maps from $S^{2}$ to $S^{2}$; the topology of the configuration space. Commun. Math. Phys. 95, 345 (1984) 
10. Taubes, C.H.: On the Yang-Mills-Higgs equations. Bull. Am. Math. Soc. 10, 295 (1984)

11. Jaffe, A., Taubes, C.H.: Vortices and monopoles. Boston: Birkhäuser 1980

12. Groisser, D.: Integrality of the monopole number in SU(2) Yang-Mills-Higgs theories on $\mathbb{R}^{3}$. Commun. Math. Phys. 93, 367-378 (1984)

13. Bogomol'nyi, E.B.: The stability of classical solutions. Sov. J. Nucl. Phys. 24, 449 (1976)

14. Taubes, C.H.: The existence of multi-monopole solutions to the non-abelian Yang-MillsHiggs equations for arbitrarily simple gauge groups. Commun. Math. Phys. 80, 343 (1981)

15. Hitchin, N.: Monopoles and geodesics. Commun. Math. Phys. 83, 579 (1982); On the construction of monopoles. Commun. Math. Phys. 89, 145 (1983)

16. Donaldson, S.K.: Nahm's equation and the classification of monopoles. Institute for Advanced Study, Princeton, Preprint (1984)

17. Segal, G.: Topology of spaces of rational functions. Acta Math. 143, 39 (1979)

18. Milnor, J.: Morse theory. Princeton: Princeton University Press 1963

19. Uhlenbeck, K.K.: Connections with $L^{p}$-bounds on curvature. Commun. Math. Phys. 83, 31 (1981)

20. Manton, N.S.: Complex structure of monopoles. Nucl. Phys. B 135, 319 (1978)

21. Coleman, S.: The magnetic monopole, fifty years later. Harvard University Preprint (1982)

22. Taubes, C.H.: Path connected Yang-Mills moduli spaces. J. Diff. Geom. (to appear)

23. Sedlacek, S.: A direct method for minimizing the Yang-Mills functional over 4-manifolds. Commun. Math. Phys. 86, 515 (1982)

24. Taubes, C.H.: The structure of static Euclidean gauge fields. Harvard University Ph.D. Thesis, 1980

25. Ladyzhenskaya, O.A.: The mathematical theory of viscous, incompressible flow. New York: Gordon \& Breach 1963

26. Morrey, C.B.: Multiple integrals in the calculus of variations. Berlin, Heidelberg, New York: Springer 1966

27. Kato, T.: Perturbation theory for linear operators. Berlin, Heidelberg, New York: Springer 1980

28. Hamilton, R.: Nash-Moser inverse function theorem. Bull. Am. Math. Soc. 7, 65 (1983)

Communicated by A. Jaffe

Received May 14, 1983; in revised form June 25, 1984 